Aus der Abteilung Urologie

(Prof. Dr. med. R-H. Ringert)

im Zentrum Chirurgie

der Medizinischen Fakultät der Universität Göttingen

\title{
Retrospektive Analyse \\ der Diagnostik und der Ergebnisse nach \\ Prostatektomie \\ bei 142 an einem Prostatakarzinom erkrankten \\ Patienten
}

\section{INAUGURAL- DISSERTATION}

zur Erlangung des Doktorgrades

der Medizinischen Fakultät

der Georg-August-Universität zu Göttingen

vorgelegt von

Gabriel Alexander Rogalli

aus

Herdecke

Göttingen 2012 
Dekan:

I. Berichterstatter:

II. Berichterstatterin:

III. Berichterstatter:

Tag der mündlichen Prüfung:
Prof. Dr. med. Frömmel

Prof. Dr. med. Ringert

Prof. Dr. med. Hille

Prof. Dr. med. Oppermann

14.Februar 2012 


\section{Inhaltsverzeichnis}

$1 \quad$ Einleitung................................................................................. 1

$1.1 \quad$ Epidemiologie ......................................................................................... 1

$1.2 \quad$ Fragestellung und Zielsetzung...............................................................2

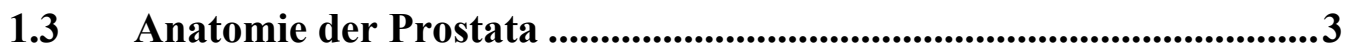

1.4 Geschichte der radikalen Prostatektomie ...........................................3

1.5 Techniken der radikalen Prostatektomie ...........................................4

1.5.1 Nervenschonendes Operationsverfahren .......................................5

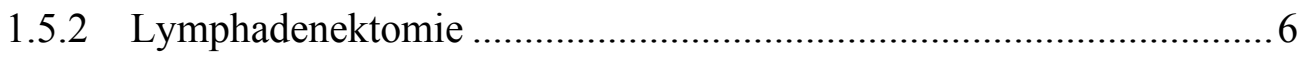

1.6 Alternative Therapiemöglichkeiten ................................................6

2 Patientengruppe und Methoden ....................................... 7

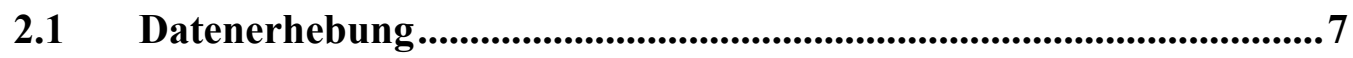

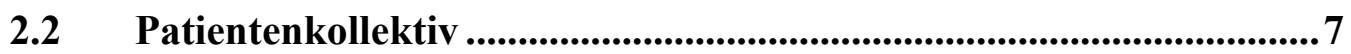

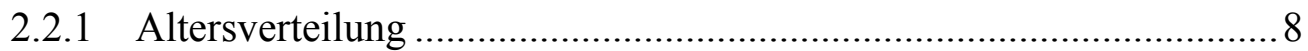

$2.3 \quad$ Histologie ................................................................................................9

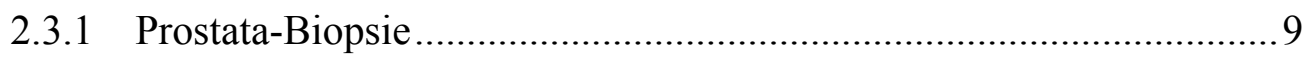

2.4 Tumorstadium nach pTNM- Klassifikation......................................9

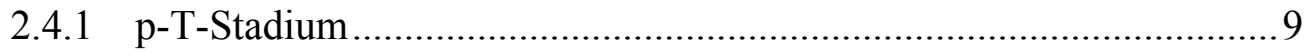

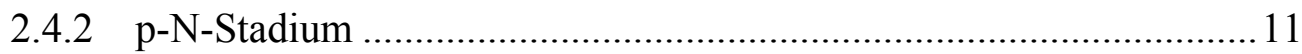

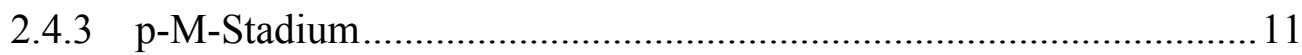

2.5 Grading nach WHO und Mostofi.................................................12

2.6 Histologisches Grading nach Gleason...............................................12 
2.7 Prostata-spezifisches Antigen (PSA) ...............................................13

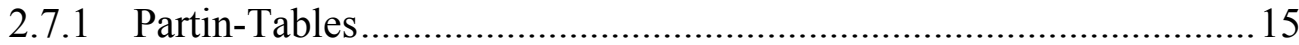

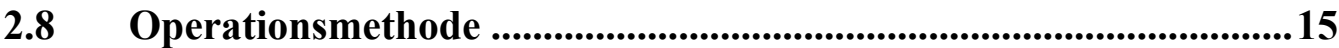

2.8.1 Nervenschonendes Operationsverfahren ...................................... 15

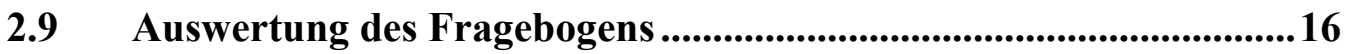

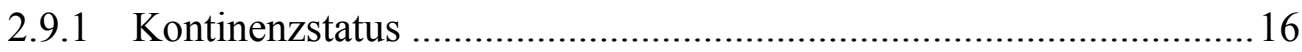

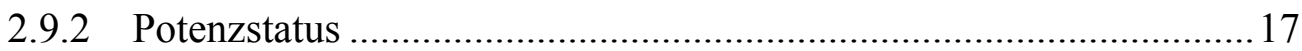

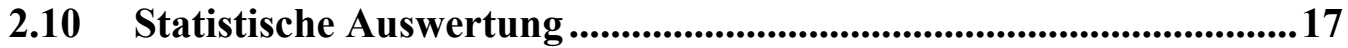

3 Ergebnisse .................................................................19

3.1 Stanzen und Prostatapräparate ..................................................19

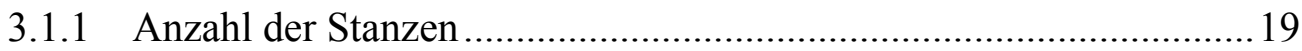

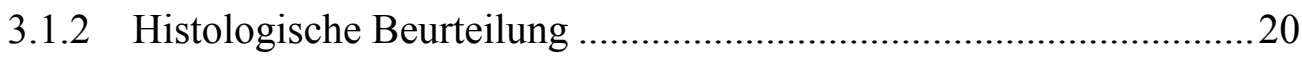

3.1.3 Sensitivität und Spezifität der Stanzbiopsien ................................22

3.1.4 Vergleich von 6 entnommenen gegenüber $>=10$ entnommenen

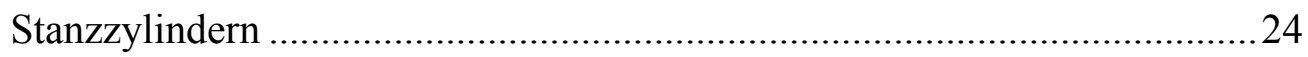

3.1.5 Sensitivität der Stanzbiopsien in Abhängigkeit von der Anzahl der

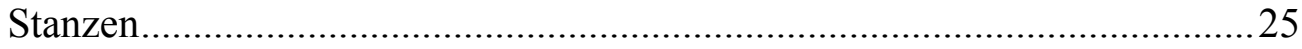

3.1.6 Analyse der Daten hinsichtlich des Absetzungsrandes der

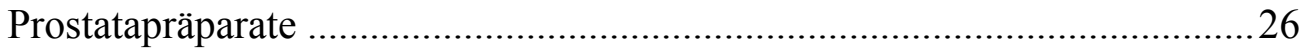

\subsection{Analyse der Daten hinsichtlich der pTNM-Klassifikation ................30}

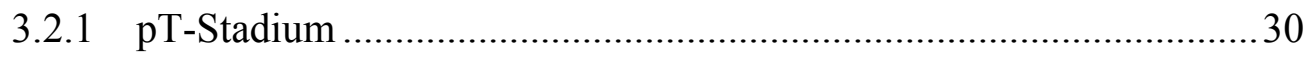

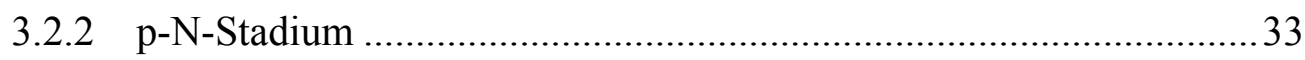

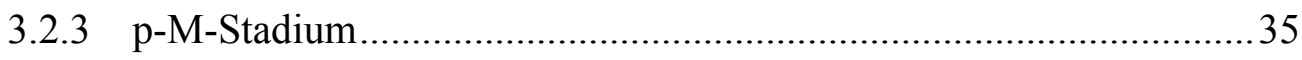

3.3 Analyse der Daten hinsichtlich des Gradings ..................................35

3.3.1 pT-Stadium in Abhängigkeit vom präoperativen Grading .................37

3.3.2 Absetzungsrand in Abhängigkeit vom präoperativen Grading ..........38 
3.3.3 Lymphknotenstatus in Abhängigkeit vom Grading. 38

3.4 Analyse der Daten hinsichtlich des Gleason-Scores 39

3.4.1 Der präoperative Gleason-Score in Abhängigkeit vom Alter. 43

3.4.2 pT-Stadium in Abhängigkeit vom Gleason-Score. 43

3.4.3 Absetzungsrand in Abhängigkeit vom Gleason-Score. 44

3.4.4 Lymphknotenstatus in Abhängigkeit vom Gleason-Score 45

3.5 Analyse der Daten hinsichtlich des präoperativen PSA-Wertes .....46

3.5.1 Einteilung der Patienten nach PSA-Wert ....................................... 46

3.5.2 pT-Stadium in Abhängigkeit vom PSA-Wert .................................48

3.5.3 Absetzungsrand in Abhängigkeit vom PSA-Wert...........................49

3.5.4 Lymphknotenbefall in Abhängigkeit vom PSA-Wert .......................50

3.6 Operationsdaten ........................................................................51

3.6.1 Anzahl der Erythrozytenkonzentrate ............................................51

3.6.2 Nervenschonende Operation....................................................... 51

3.7 Ergebnisse der Patientenbefragung .....................................................52

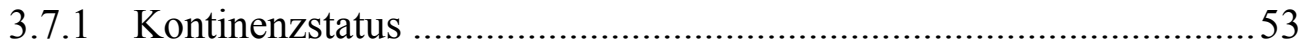

3.7.2 Lebensqualität im Bezug auf den Kontinenzstatus...........................57

3.7.3 Weitere die Kontinenz beeinflussende Faktoren ............................58

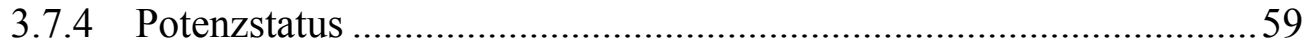

4 Diskussion ...................................................................... 63

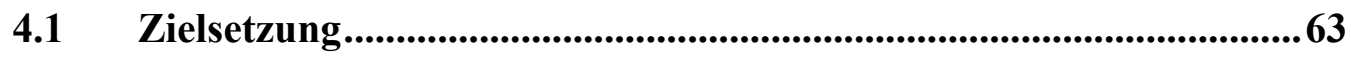

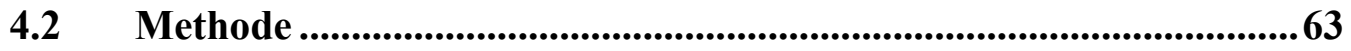

4.3 Histologie der Stanzen und Prostatapräparate.................................64

4.4 Positiver Absetzungsrand (Radikalität) ...........................................69

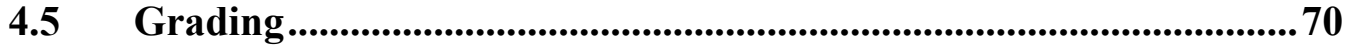


4.6 Prä- und postoperativer Gleason ...........................................................71

4.7 Konsequenzen der radikalen Prostatektomie .....................................74

4.8 Konsequenzen und Ausblick.............................................................76

$5 \quad$ Zusammenfassung.............................................................. 77

6 Anhang ................................................................................... 78

$7 \quad$ Literaturverzeichnis............................................................85 


\section{Einleitung}

\subsection{Epidemiologie}

In Deutschland werden derzeit jährlich etwa 48.650 Prostatakarzinome diagnostiziert. So ist die Prostata mit 22,3\% die häufigste Lokalisation bösartiger Neubildungen beim Mann. Bei den zum Tode führenden Krebserkrankungen steht das Prostatakarzinom mit 10,4\% an dritter Stelle. Die Sterberaten sind seit 1970 nahezu unverändert (Bertz et al. 2006). Weltweit ist das Prostatakarzinom das vierthäufigste Karzinom des Mannes.

Die Ätiologie des Prostatakarzinoms ist nicht endgültig geklärt. Die einzig sicher anerkannten Risikofaktoren sind Alter, Rasse, fettreiche Ernährung und eine positive Familienanamnese (Bas et al. 2003). Als Hauptursachen werden genetische und Umweltfaktoren angegeben (Reiter et al. 2002). Das Prostatakarzinom ist vornehmlich eine Erkrankung des älteren Mannes. 75\% der Erstdiagnosen werden bei Männern über 65 Jahren gestellt. Die Inzidenz der diagnostizierten Prostatakarzinome im Alter von 50 bis 59 Jahren ist seit 1970 maßgeblich gestiegen. Diese Tendenz ist auf die steigende Anzahl, Anwendung und bessere Qualität von diagnostischen Maßnahmen zurückzuführen (Reiter et al. 2002).

Diese epidemiologischen Daten belegen eindrucksvoll, welche Bedeutung den Vorsorgeuntersuchungen, der Diagnostik und der Therapie des Prostatakarzinoms zukommt. Die heute beim lokal begrenzten Prostatakarzinom bevorzugt durchgeführte radikale retropubische Prostatektomie bringt allerdings im postoperativen Verlauf oft typische Komplikationen mit sich. Diese können die Lebensqualität der Patienten entscheidend beeinflussen. Am häufigsten treten folgende Komplikationen auf: Zum einen die erektile Dysfunktion, die auch bei einem nervenschonenden Operationsverfahren nicht ausgeschlossen ist. Als weitere Komplikation ist die Harninkontinenz zu nennen, die sich für die Patienten häufig stark einschränkend auf das Alltagsleben auswirkt. Nicht zuletzt spielen auch evtl. Rezidive und Nachbehandlungen eine wichtige Rolle. 
Angesichts der kurativen Therapie des Prostatakarzinoms können die Komplikationen nach der Operation zunächst in den Hintergrund treten. Gibbons formulierte den Grundsatz: Chirurgische Therapie ist nur kurativ, wenn der ganze Tumor entfernt wird. Chirurgische Therapie ist nur notwendig, wenn das Karzinom eine höhere Morbidität verursachen oder die Lebenserwartung des Patienten reduzieren würde (Gibbons 2002).

Bei der vorliegenden Studie handelt es sich um eine retrospektive Datenauswertung und Befragung von 142 Patienten, die in der Zeit vom 01.01.2000 bis zum 31.12.2003 in der Abteilung für Urologie des Universitätsklinikums Göttingen aufgrund eines Prostatakarzinoms einer radikalen retropubischen Prostatektomie unterzogen worden sind. Es wurden Daten von der präoperativen Diagnostik, über die Operation bis zu einem Follow-up von durchschnittlich 4 Jahren und 10,5 Monaten erhoben und ausgewertet. Ein besonderes Augenmerk galt hierbei der prä- und postoperativen Histologie und deren Vergleich, dem intraoperativen Verlauf, den im postoperativen Zeitraum aufgetretenen Nebenwirkungen und deren Auswirkungen auf die Lebensqualität der Patienten.

\subsection{Fragestellung und Zielsetzung}

Ziel der Arbeit ist es zu zeigen, ob die Ausbreitung des Prostatakarzinoms mit Hilfe der diagnostischen Möglichkeiten wie der Bestimmung des präoperativen PSA-Wertes und der histopathologischen Untersuchung der aus dem Organ entnommenen Stanzbiopsien festzustellen und ein passendes Therapiekonzept auf dieser Basis festzulegen ist. Eine radikale Prostatektomie ist nur dann als kurative Therapie anzusehen, wenn das Karzinom auf das Organ begrenzt ist.

Weiterhin sollen klinische Aspekte, wie die postoperative Kontinenz, die postoperative Erektionsfähigkeit, insbesondere im Hinblick auf den Erfolg des nervenschonenden Operationsverfahrens, sowie die Auswirkungen eines solchen Eingriffes auf die Lebensqualität der Patienten beleuchtet werden. 


\subsection{Anatomie der Prostata}

Die Prostata ist eine exokrine Drüse von etwa der Größe einer Esskastanie. Im dritten Embryonalmonat entwickelt sie sich aus zahlreichen Aussprossungen der Pars prostatica der Urethra in das umgebende Mesenchym. Sie liegt zwischen Harnblasenfundus und Diaphragma urogenitale fest im Bindegewebe verankert, beiderseits flankiert von den Levatorschenkeln. Vom Rektum aus ist der hintere Teil der Prostata tastbar. Rechter und linker Prostatalappen legen sich um die Harnröhre und werden vor dieser durch den Isthmus prostatae verbunden.

\subsection{Geschichte der radikalen Prostatektomie}

Die radikale Prostatektomie als ein effektives Verfahren zur Therapie des lokalen Prostatakarzinoms findet ihren Ursprung vor fast 150 Jahren. Bereits 1858 beschrieb Kuchler die Operationstechnik des perinealen Zugangswegs (Kuchler 1866). Die ersten zwei radikalen Prostatektomien über den perinealen Zugangsweg führte 1867 der Chirurg Billroth in Wien durch (Nöske und Breiwieser 1973).

1905 veröffentlichte der Chirurg Young aus Baltimore in einer umfassenden Studie 40 Fälle nach radikaler perinealer Prostatektomie. Er kam zu dem Ergebnis, dass Heilung nur bei radikaler Anwendung, wie der routinemäßigen Entfernung der Samenblasen, der Vasa deferentia und dem größten Teil des Harnblasendreiecks zusammen mit der vollständigen Prostata erwartet werden kann (Young 1905). Hierdurch etablierte er den perinealen Zugangsweg als routinemäßiges Verfahren.

Einen Meilenstein in der Geschichte der radikalen Prostatektomie legte 1945 Millin. Er führte das Verfahren des retropubischen Zugangswegs ein (Millin 1947). Diese Methode hat den Vorteil, dass sie die Möglichkeit zur gleichzeitigen pelvinen Lymphknoten- Dissektion bietet und das Rektum intraoperativ seltener verletzt wird als bei dem perinealen Zugang. Bis heute ist die radikale retropubische Prostatektomie die bevorzugte Therapie bei Patienten mit lokalem Prostatakarzinom.

$\mathrm{Ab}$ Mitte des 20. Jahrhunderts wurden einige technische Details durch Ansell (1959), Campbell (1959) und andere ergänzt. Hierzu ist besonders Walsh hervorzuheben. Er beschrieb nach Studien an anatomischen Präparaten den venösen $\mathrm{Ab}$ - 
fluss aus dem Penis und entwickelte 1979, zusammen mit dem Chirurgen Reiner eine Methode, um diesen Venenkomplex im Gebiet der Prostata bei radikaler Prostatektomie zu schonen. Dies minimierte den intraoperativen Blutverlust und verbesserte dadurch die Sicht des Operateurs auf ein blutärmeres Operationsfeld (Reiner und Walsh 1979). Wenig später beschrieb er zusammen mit Donker den autonomen pelvinen Nervenplexus und dessen Fasern zu den Corpora cavernosa des Penis. Sie entwickelten eine Methode, diese Nervenfasern zu schonen, wodurch die potenzerhaltende Operation ermöglicht wurde (Walsh und Donker 1982). Das Verständnis der periprostatischen Anatomie hat schließlich die Morbidität nach radikaler Prostatektomie signifikant gesenkt (Eastham et al. 1996). Zur Behandlung des lokal begrenzten Prostatakarzinoms ist somit die radikale retropubische Prostatektomie zu einem standardisierten, routinemäßigen Eingriff mit geringer Mortalität geworden (Frohmüller und Wirth 2002).

Ende der 1980-er Jahre hielt mit der Laparoskopie eine neue Technik Einzug in die Chirurgie. Die erste laparoskopische radikale Prostatektomie (LRP) führten Schuessler et al. in den frühen 1990-er Jahren durch. Sie hielten diese Methode aber nicht für eine konkurrenzfähige Alternative (Schuessler et al. 1997). Eine verbesserte Technik der Instrumente erlaubte einigen Zentren jedoch wenig später die Methode zu verfeinern und Operationszeiten zu verkürzen (Abbou et al. 2000, Guillonneau und Vallancien 2000). Die laparoskopische Technik ist inzwischen gut durchführbar und die zur Verfügung stehenden Ergebnisse sind vergleichbar mit denen der radikalen retropubischen Prostatektomie. Es müssen jedoch auch die onkologischen Langzeitergebnisse vergleichbar sein und signifikante Vorteile in der postoperativen Morbidität erst nachgewiesen werden (Winfield und Cadeddu 2002).

\subsection{Techniken der radikalen Prostatektomie}

Zur Therapie des lokalen Prostatakarzinoms durch radikale Prostatektomie gibt es im Wesentlichen drei Techniken. Die perineale, die retropubische und die, in einigen speziellen Zentren durchgeführte, laparoskopische radikale Prostatektomie.

Der perineale Zugang, von Young 1905 erstmals beschrieben und durchgeführt, wurde von Belt popularisiert. Dieser postulierte (Seite 494): „Wegen des Zugangs, der Ordnungsmäßigkeit der Hämostase, visueller Kontrolle in allen Phasen, 
schneller Anpassung an individuelle anatomische Variationen, starkem Rückgang von Schockzuständen, schneller Heilung und kurzem Krankenhausaufenthalt, " ist die radikale perineale Prostatektomie eine effektive chirurgische Methode mit vielen Vorteilen (Belt et al. 1939). Diese Feststellung ist noch heute aktuell und ist für die steigende Anzahl von Patienten mit kleinen, nicht palpablen Karzinomen, die nur durch den Anstieg des PSA diagnostiziert werden, eine angemessene Therapieoption (Gibbons 2002). Ein wesentlicher Nachteil dieses Zugangs ist, dass die Möglichkeit der gleichzeitigen pelvinen Lymphknotendissektion nicht besteht. Zudem ist der Zugangsweg relativ schmal (Frohmüller 1991).

Die Langzeitergebnisse der laparoskopischen Technik sind aufgrund ihrer jungen Geschichte noch nicht endgültig beurteilbar. Außer der niedrigeren Bluttransfusionsrate sind bisher noch keine signifikanten Vorteile der LRP gegenüber der radikalen retropubischen Prostatektomie bekannt (Winfield und Cadeddu 2002). Roumeguere et al. stellten fest, dass Patienten nach offener Prostatektomie ihre Kontinenz schneller zurück erlangen als laparoskopisch behandelte Patienten (Roumeguere et al. 2003). Die onkologischen Ergebnisse und die niedrige Morbidität bei der nervenschonenden RRP sind ein hoher Standard, den die laparoskopische Technik erst einmal überbieten muss (Winfield und Cadeddu 2002).

Der retropubische Zugang wird nochmals unterteilt in die aszendierende und die deszendierende Technik. Bei letzterer wird zunächst mit der Präparation der Prostata am Blasenhals begonnen; zuletzt wird die Harnröhre durchtrennt. Diese Methode wurde von Campbell beschrieben (Campbell 1959).

Bei der aszendierenden Technik wird die Prostata von kaudal nach kranial freipräpariert, wobei die Harnröhre relativ früh durchtrennt wird. Hier hat Walsh die entscheidenden Grundlagen etabliert (Walsh 2002).

\subsubsection{Nervenschonendes Operationsverfahren}

Bei Patienten mit einem niedrigen Tumorstadium bis pT2b kann das nervenschonende Operationsverfahren empfohlen werden (Shah et al. 2003). Kenntnisse über die präoperative erektile Funktion, das Alter des Patienten und den antizipierten Grad der Erhaltung des neurovaskulären Bündels (Walshes Bündel) helfen bei der Einschätzung der erektilen Funktion nach radikaler Prostatektomie (Eastham et al. 
2002). Bei dieser Methode besteht allerdings ein höheres Risiko für einen positiven Absetzungsrand beziehungsweise für Rezidive des Karzinoms. Deshalb sollten Kandidaten für das nervenschonende Operationsverfahren sorgfältig ausgesucht werden (Aus et al. 2005).

\subsubsection{Lymphadenektomie}

Die pelvine Entnahme der Lymphknoten während der Operation findet unmittelbar vor der Entfernung der Prostata statt. Sie werden histopathologisch nach dem TNM-System eingestuft. Mittels dieser Lymphadenektomie wird die lymphogene Metastasierung bestimmt.

\subsection{Alternative Therapiemöglichkeiten}

Als weiteren kurativen Therapieansatz gibt es neben der radikalen Prostatektomie die Möglichkeit der externen oder internen Radiotherapie. Prostatakarzinome mit einem niedrigen Malignitätsgrad und/oder in einem frühen Stadium, die Männer betreffen, deren Lebenserwartung aufgrund ihres Lebensalters nicht sehr hoch ist, können unter engmaschiger Kontrolle überwacht werden (active surveillance). Eine weitere palliative Therapiemaßnahme ist die Möglichkeit der hormonellen Manipulation des Prostatakarzinoms. 


\section{Patientengruppe und Methoden}

\subsection{Datenerhebung}

Die Daten zur präoperativen-, intraoperativen- und postoperativen Situation wurden durch die routinemäßigen Vor- und Nachuntersuchungen erhoben und retrospektiv der Dokumentation aus den Kranken- bzw. Nachsorgeakten entnommen. Die Patienten wurden postalisch mit einem Fragebogen kontaktiert. Anhand des validierten Fragebogens „Fragebogen zur sexuellen Gesundheit beim Mann“(IIEF-5) und weiteren teilweise validierten Fragen zum Kontinenzstatus und zur Lebensqualität wurden die präoperative-, die zeitnahe postoperative und die aktuelle Situation erhoben. Zudem wurde der zuletzt bekannte PSA-Wert, sowie eine eventuelle adjuvante Therapie, der sich der Patient unterzogen haben könnte, abgefragt.

Der vollständige Fragebogen ist im Anhang beigefügt.

\subsection{Patientenkollektiv}

Insgesamt entsprachen 142 Patienten den Kriterien der Studie. Hierbei handelt es sich um alle Patienten, die zwischen dem 01.01.2000 und dem 31.12.2003 in der urologischen Abteilung des Universitätsklinikums Göttingen wegen eines Prostatakarzinoms radikal retropubisch prostatektomiert wurden.

Von den 142 Patienten haben sich 104 (73,3\%) bereit erklärt, in vollem Umfang an der Studie teilzunehmen. Dies beinhaltet die Einwilligung zur Einsicht in ihre Krankenakten und die Verwendung der entnommenen anonymisierten Daten, sowie die Beantwortung des Fragebogens. 2 Patienten $(1,4 \%)$ haben die Verwendung ihrer Daten vollständig abgelehnt, 32 Patienten (22,4\%) haben es abgelehnt, den Fragebogen zu beantworten und 4 Patienten (2,8\%) sind inzwischen verstorben (Tab.1). 
Anzahl der Patienten

142

$100 \%$

Datenverwendung abgelehnt

$2 \quad 1,4 \%$

Fragebogen abgelehnt

32

$22,5 \%$

Später verstorben

4

$2,8 \%$

Vollständige Daten

104

$73,3 \%$

Tab.1: Zusammensetzung des Patientenkollektivs im Bezug auf die Beantwortung des Fragebogens und die Datenverwendung

\subsubsection{Altersverteilung}

Das mittlere Alter der teilnehmenden Patienten betrug zum Zeitpunkt der Operation 64,4 Jahre, bei einem Median von 65 Jahren. Der jüngste Patient war 44, der älteste 81 Jahre alt (Abb. 1).

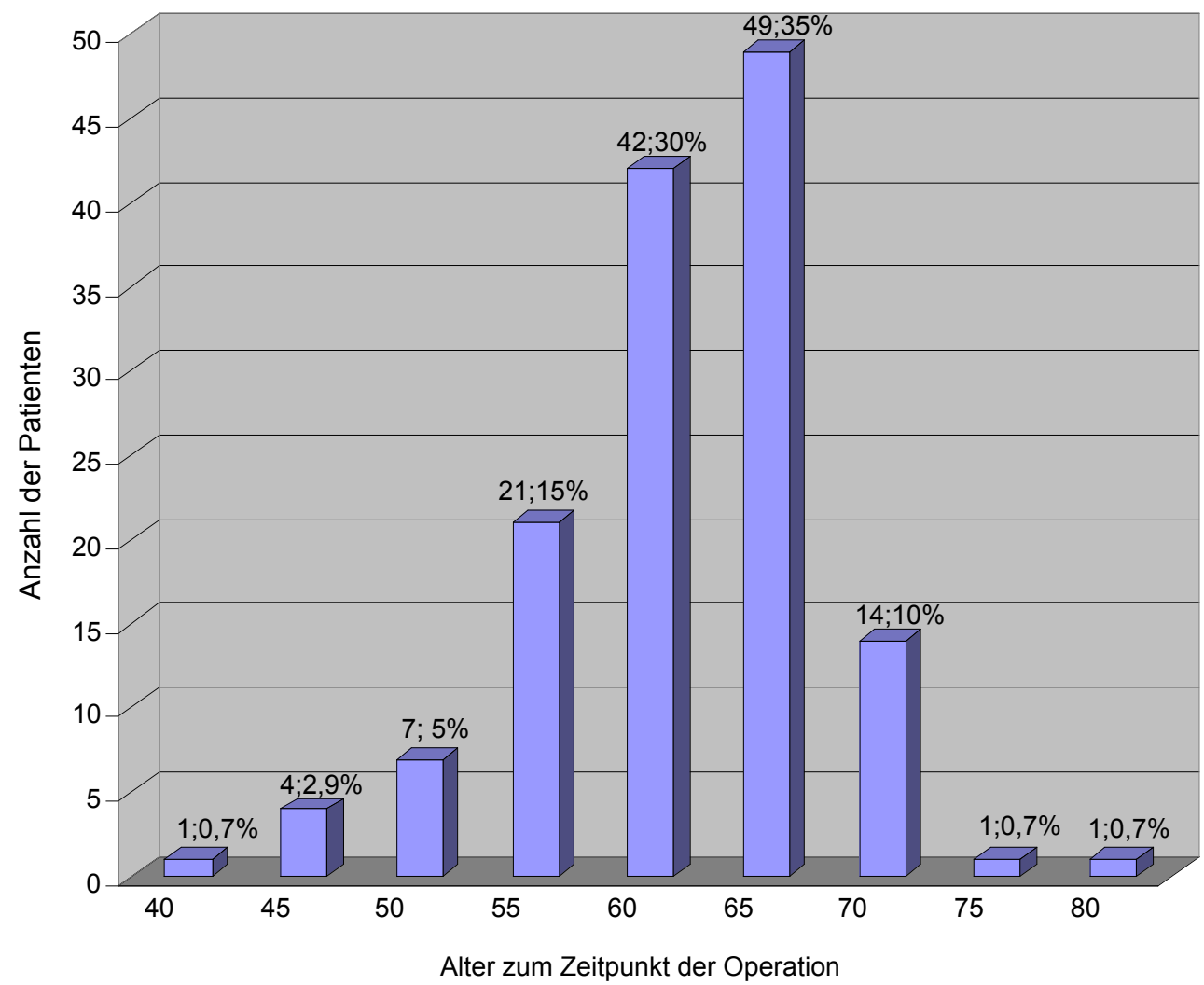

Abb.1: Verteilung des Alters des Patientenkollektivs 


\subsection{Histologie}

Die jeweilige histopathologische Befunderhebung der präoperativ entnommenen Stanzbiopsien erfolgte zum größten Teil in der Abteilung für allgemeine Pathologie des Universitätsklinikums Göttingen, zum Teil auch an anderen pathologischen Instituten. Die Beurteilung der intraoperativ entnommenen Lymphknoten, sowie des entfernten Prostatagewebes fand ausschließlich in der Pathologie des Universitätsklinikums Göttingen statt.

Die Präparate wurden jeweils nach pTNM-Klassifikation, Malignitätsgrad G und ab Mitte des Jahres 2000 auch nach dem Gleason-Score beurteilt.

\subsubsection{Prostata-Biopsie}

Um das Prostatakarzinom definitiv zu sichern, ist eine Biopsie des Organs obligatorisch.

Üblicherweise wird die Probenentnahme mit einer Sextantenbiopsie transrektal unter Ultraschallkontrolle durchgeführt. Es werden jeweils drei Stanzen aus dem rechten und linken Prostatalappen entnommen, um unter anderem festzustellen, ob einer oder beide Seitenlappen der Prostata von der karzinomatösen Veränderung betroffen sind. Zusätzlich werden palpatorisch oder sonographisch auffällige Areale der Prostata gezielt biopsiert. In der Regel wurden die Biopsien im Klinikum Göttingen entnommen. Bei einigen Patienten wurde die Diagnostik in peripheren Krankenhäusern oder bei niedergelassenen Urologen durchgeführt. Hierbei wurden teilweise auch weniger Stanzen entnommen oder auch Aspirationszytologien mittels Feinnadelbiopsie erstellt.

\subsection{Tumorstadium nach pTNM- Klassifikation}

Die Klassifizierung des Prostatakarzinoms erfolgt nach dem von der UICC im Jahre 2003 festgelegten TNM-System für maligne Tumoren (Schmitz-Dräger et al. 2003). Die Daten wurden teilweise vor dem Jahr 2003 erhoben und mussten nach der neuen Klassifizierung entsprechend aktualisiert werden.

\subsection{1 p-T-Stadium}

Die Einteilung der Tumoren erfolgte nach den in Tabelle 2 aufgeführten Kriterien. 


\section{T- Primärtumor}

TX Primärtumor kann nicht beurteilt werden

T0 Kein Anhalt für Primärtumor

T1 Klinisch nicht erkennbarer Tumor, der weder tastbar noch in bildgebenden Verfahren sichtbar ist

T1a Tumor zufälliger histologischer Befund (,incidental carcinoma“) in $5 \%$ oder weniger des resezierten Gewebes

T1b Tumor zufälliger histologischer Befund (,incidental carcinoma“) in mehr als 5\% des resezierten Gewebes

T1c Tumor durch Nadelbiopsie diagnostiziert (z.B. wegen erhöhtem PSA)

T2 Tumor begrenzt auf die Prostata (1)

T2a Tumor befällt die Hälfte eines Lappens oder weniger

T2b Tumor befällt mehr als die Hälfte eines Lappens

T2c Tumor in beiden Lappen

T3 Tumor durchbricht die Prostatakapsel (2)

T3a Extrakapsuläre Ausbreitung (einseitig oder beidseitig)

T3b Tumor infiltriert Samenblase(n)

T4 Tumor ist fixiert oder infiltriert andere benachbarte Strukturen als Samenblasen, z.B. Blasenhals, Sphincter externus, Rectum, und/oder ist an Beckenwand fixiert

\section{Anmerkungen:}

1 Ein Tumor, der durch Nadelbiopsie in einem oder beiden Lappen gefunden wird, aber weder tastbar noch in bildgebenden Verfahren sichtbar ist, wird als T1c klassifiziert;

2 Invasion in den Apex der Prostata oder in die Prostatakapsel (aber nicht darüber hinaus) wird als T2 (nicht T3) klassifiziert

Tab.2: TNM-Klassifikation des Prostatakarzinoms (Schmitz-Dräger et al. 2003, S.1252) 


\subsection{2 p-N-Stadium}

Die bei der pelvinen Lymphadenektomie entnommenen Lymphknoten werden nach den Kriterien in Tabelle 3 eingeteilt.

\section{N- regionäre Lymphknoten}

NX Regionäre Lymphknoten können nicht beurteilt werden

N0 Keine regionären Lymphknotenmetastasen

N1 Regionäre Lymphknotenmetastasen

Anmerkungen:

Regionäre Lymphknoten sind die Lymphknoten des kleinen Beckens, die im Wesentlichen den Beckenlymphknoten unter der Bifurkation der Aa. Iliacae communes entsprechen. Die Seitenlokalisation beeinflusst die N-Klassifikation nicht.

Tab.3: TNM-Klassifikation des Prostatakarzinoms (Schmitz-Dräger et al. 2003, S.1254)

\subsection{3 p-M-Stadium}

Die Kriterien zur Einteilung von Metastasen sind in Tabelle 4 aufgeführt.

\section{M- Fernmetastasen}

MX Fernmetastasen können nicht beurteilt werden

M0 Keine Fernmetastasen

M1 Fernmetastasen

M1a Nichtregionäre(r) Lymphknoten

M1b Knochen

M1c Andere Lokalisation(en)

Anmerkungen:

Wenn Metastasen in mehr als einer Lokalisation nachweisbar sind, soll die höchste Kategorie benutzt werden

Tab.4: TNM-Klassifikation des Prostatakarzinoms (Schmitz-Dräger et al. 2003, S.1256) 


\subsection{Grading nach WHO und Mostofi}

Bei diesem System stehen der histologisch/zytologische Atypie- und der histologische Differenzierungsgrad im Vordergrund.

Die Atypie oder Anaplasie charakterisiert Mostofi durch Vorliegen von Kernvariationen und Veränderungen in der Chromatinverteilung.

Die Differenzierung entspricht der Ausbildung von Drüsen, wobei undifferenzierte Tumoren demnach mit dem Verlust von Drüsen gleichgesetzt werden.

Das Gradingsystem von Mostofi ist von der WHO übernommen worden (Mostofi et al. 1980, Helpap 1998) (Tab.5)

\begin{tabular}{|l|l|}
\hline Gx & $\begin{array}{l}\text { Differenzierungsgrad kann nicht be- } \\
\text { stimmt werden }\end{array}$ \\
\hline G1 & $\begin{array}{l}\text { gut differenziert } \\
\text { leichte Anaplasie }\end{array}$ \\
\hline G2 & mäßig differenziert \\
mäßige Anaplasie \\
\hline G3 & schlecht differenziert/ undifferenziert \\
& ausgeprägte Anaplasie \\
\hline
\end{tabular}

Tab. 5: Übersicht des histopathologischen Gradings (Schmitz-Dräger et al. 2003)

\subsection{Histologisches Grading nach Gleason}

Grundlage des Gradingsystems nach Gleason sind die verschiedenen Wachstumsmuster des Prostatakarzinoms.

Im Gewebe der Prostata kommt es bei einem karzinomatösen Befall zu einem Verlust der histologischen Architektur. Bei den befallenen Zellen werden verschiedene Wachstumsmuster unterschieden, wobei jeweils ein primär und ein sekundär vorherrschendes Muster bestimmt wird. Je nach Grad der Abnahme der histologischen Differenzierung werden die Wachstumsmuster durch 1 bis 5 Punkte graduiert. 
Der höchste Malignitätsgrad wird demnach sowohl im primären als auch im sekundären Muster mit fünf Punkten charakterisiert $(5+5=10)$. Dementsprechend kennzeichnet ein Punkt in beiden Grundmustern den niedrigsten Malignitätsgrad $(1+1=2)$.

\subsection{Prostata-spezifisches Antigen (PSA)}

Der entscheidende Schritt in der Entwicklung der Prostatakarzinomdiagnostik war die Entdeckung des prostataspezifischen Antigens (PSA) durch Wang (Wang et al. 1979). Das PSA ist ein Glykoprotein, welches fast nur in den Epithelzellen der Prostata gefunden wird (Armbruster 1993). Der Anstieg des PSA im Serum zeigt bis auf wenige Ausnahmen eine Erkrankung der Prostata an (Carter und Partin 2002).

Bei der Bestimmung des PSA wird die Serumkonzentration gemessen. Die europäische und amerikanische weiße Bevölkerung weist einen physiologischen PSAWert von $0,4 \mathrm{ng} / \mathrm{ml}$ im Serum auf (Preston et al. 2000).

Zu einem Anstieg des PSA-Wertes kann es bei benigner Prostatahyperplasie, akuter Prostatitis, nach therapeutischen und diagnostischen Eingriffen wie beispielsweise bei Urethroskopien, Zystoskopien, transrektalen Ultraschalluntersuchungen (TRUS) und digitalen rektalen Untersuchungen (DRE) und eben beim Prostatakarzinom kommen. Das PSA ist somit ein organ- und gewebsspezifischer, jedoch kein tumorspezifischer Marker (Oesterling et al. 1993). Ist die PSAKonzentration im Serum erhöht, so ist dieser Wert nicht als definierte absolute Messgröße zu interpretieren, sondern als relative Richtlinie für das Vorliegen eines pathologischen Zustands (Rifkin 1998).

Durch die routinemäßigen PSA-Bestimmungen im Rahmen von Vorsorgeuntersuchungen wird das Prostatakarzinom weitaus früher entdeckt als zuvor. Die meisten Tumore werden bereits im lokalen Stadium (pT1, pT2) erkannt. Das Auftreten von lokal fortgeschrittenen Stadien (pT3, pT4), bei denen das maligne Wachstum die Prostatakapsel überschritten hat, ist seltener geworden (Catalona 2003).

Als Normwert für den Übergang in den pathologischen Bereich ist die obere Grenze auf $4 \mathrm{ng} / \mathrm{ml}$ festgelegt worden. Ab diesem Wert wird eine Biopsie der Prostata empfohlen (Denis et al. 1995). Allerdings gibt es Studien, bei denen in 
bis zu 20\% der Fälle ein Prostatakarzinom bei einem PSA-Wert unter 4 ng/ml nachgewiesen werden konnte (Catalona et al. 2000, Rifkin 1998).

Der PSA- Spiegel kann durch das Lebensalter beeinflusst werden. So kann beispielsweise eine benigne Prostatahyperplasie, eine Erkrankung des älteren Mannes, zu einem Anstieg des PSA-Wertes führen. Oesterling und seine Mitarbeiter stellten aufgrund von Ergebnissen aus Reihenuntersuchungen, positiven Korrelationen des PSA-Wertes mit dem klinischen Stadium und der pathohistologischen Untersuchung des Prostatapräpatates nach der radikalen Prostatektomie fest, dass die Konzentration des PSA im Serum direkt mit dem Alter und dem Volumen der Prostata korreliert, welches auch direkt vom Alter abhängig ist (Oesterling et al. 1993). Anhand dieser Erkenntnisse werden altersspezifische Grenzwerte ermittelt (Tab. 6)

\begin{tabular}{|c|c|}
\hline Alter der Patienten & PSA ng/ml \\
\hline $40-49$ & $0,0-2,5$ \\
\hline $50-59$ & $0,0-3,5$ \\
\hline $60-69$ & $0,0-4,5$ \\
\hline$>70$ & $0,0-6,5$ \\
\hline
\end{tabular}

Tab.6: Empfohlene PSA- Grenzwerte in Abhängigkeit vom Lebensalter (Oesterling et al. 1993)

Die Ausbreitung des Tumors korreliert mit dem Anstieg des PSA- Wertes im Serum. Dem entsprechend kann man anhand des PSA-Spiegels das Risiko des Auftretens von Lymphknotenmetastasen berechnen (Denis et al. 1995) (Tab.7).

\begin{tabular}{|c|c|}
\hline PSA ng/ml & Lymphknoten positiv (pN+) \\
\hline $0-4$ & $1-8 \%$ \\
\hline $4-10$ & $5-12 \%$ \\
\hline $10-20$ & $16-18 \%$ \\
\hline$>20$ & $25-74 \%$ \\
\hline
\end{tabular}

Tab.7: Risiko von Lymphknotenmetastasen in Abhängigkeit von der Höhe des präoperativ im Serum bestimmten PSA (Denis et al. 1995) 


\subsubsection{Partin-Tables}

Um nicht nur den Lymphknotenstatus, sondern auch eine extraprostatische Ausbreitung oder eine Samenblaseninfiltration des Tumors abschätzen zu können, haben Partin et al. Nomogramme entwickelt. Aus diesen, als „Partin-Tables“ bekannten Tabellen lässt sich bei Kenntnis des PSA-Spiegels und des histologischen Differenzierungsgrades nach Gleason die Ausbreitung des Tumors abschätzen. Die erstmalig 1984 erschienenen Nomogramme beruhen auf einer multinominalen retrospektiven Analyse von Patientendaten und wurden von Partin et al. im Laufe der Zeit immer wieder verbessert und den aktuellen Entwicklungen angepasst. Sie sollen der individuellen Beratung von Patienten dienen und ihnen bei wichtigen Entscheidungen hinsichtlich ihrer Erkrankung helfen (Partin et al. 2001).

\subsection{Operationsmethode}

Alle 142 Patienten sind auf dem retropubischen Zugangsweg radikal prostatektomiert worden.

\subsubsection{Nervenschonendes Operationsverfahren}

Bei diesem Verfahren gilt es, die Blutgefäße mit den Nervi erigentes, welche hauptsächlich die glatte Muskulatur der Schwellkörper des Penis versorgen und unmittelbar dorsolateral der Prostatakapsel verlaufen, zu belassen. Ziel dieser Methode ist es, die Möglichkeit einer ausreichenden Erektion für Geschlechtsverkehr zu erhalten. Zahlen in der aktuellen Literatur belegen, dass $76 \%$ der vor der Operation potenten Männer nach bilateraler und 53\% nach unilateraler oder partieller nervenschonenden Operation eine ausreichende Erektion für Geschlechtsverkehr hatten (Kundu et al. 2004).

In unserem Patientenkollektiv wurde nur bei einseitigem Tumorbefall der Prostata ein solches Verfahren in Erwägung gezogen werden. Weitere Entscheidungsgrundlagen waren die Höhe des PSA-Wertes, der Gleason-Score, die erektile Funktion des Patienten vor der Operation und das Lebensalter. 


\subsection{Auswertung des Fragebogens}

\subsubsection{Kontinenzstatus}

Zur Abschätzung des Kontinenzstatus hat sich folgende Einteilung bewährt (Tab.8):

\begin{tabular}{|l|l|}
\hline Absolut Kontinent Grad 0 & Kein Harnverlust, keine Vorlage \\
\hline Inkontinenz Grad I & $\begin{array}{l}\text { Harnverlust bei Niesen, Husten, } \\
\text { Lachen usw. }\end{array}$ \\
\hline Inkontinenz Grad II & $\begin{array}{l}\text { Harnverlust bei leichter körperlicher } \\
\text { Anstrengung, wie z.B. Treppenstei- } \\
\text { gen, Gehen usw. }\end{array}$ \\
\hline Inkontinenz Grad III & $\begin{array}{l}\text { Unkontrollierter Harnabgang im } \\
\text { Stehen und Liegen }\end{array}$ \\
\hline Inkontinenz Grad IV & Harnverlust tritt ständig auf \\
\hline
\end{tabular}

Tab.8: Grade der Inkontinenz

Zur weiteren Validierung wurden noch die Anzahl der Vorlagen, die Häufigkeit und Menge des Harnverlustes und der eventuelle Gebrauch von Hilfsmitteln, wie Medikamente oder Katheter, erfragt.

Um die Auswirkungen der Prostatektomie auf die Kontinenz genau einschätzen zu können, wurden der Kontinenzstatus zu den Zeitpunkten vor, kurz nach der Operation und im Follow-up erfragt. 
Der Einfluss auf die Lebensqualität des einzelnen Patienten, der durch Inkontinenz auftreten kann, wurde über eine Skala zur Selbsteinschätzung der Belastung durch eine eventuelle Inkontinenz erhoben.

\subsubsection{Potenzstatus}

Um den Potenzstatus zu erheben, kam der validierte „Fragebogen zur sexuellen Gesundheit beim Mann“(IIEF-5) zum Einsatz. Es wurde auch hier der Status zu den Zeitpunkten vor, kurz nach der Operation und im Follow-up erfragt.

Die fünf Fragen des verkürzten Fragebogens haben für die Unterscheidung von Patienten mit erektiler Dysfunktion von denen ohne erektile Dysfunktion eine Sensitivität von 98\% und eine Spezifität von 88\% (Rosen 1999).

Es ist eine Maximalpunktzahl von 25 erreichbar. Wenn der Patient in der Summe der fünf Fragen weniger als 22 Punkte erreicht, bestehen bei ihm Anzeichen für eine erektile Dysfunktion.

\subsection{Statistische Auswertung}

Statistische Testverfahren werden eingesetzt, um zu untersuchen, ob die zufallsbedingte Streuung der Daten ausreicht, einen beobachteten Effekt zu erklären. Bei den statistischen Fragestellungen in dieser Arbeit wurde jeweils die Hypothese aufgestellt, dass in der Grundgesamtheit kein Effekt existiert (sog. Nullhypothese). Wenn die Wahrscheinlichkeit gering ist, dass das Ergebnis sich zufällig ergeben haben könnte, wird die Hypothese verworfen und das Ergebnis als statistisch signifikant bezeichnet. Wenn die statistische Berechnung ergibt, dass das Ergebnis zufällig zustande kommt, gilt dieses als statistisch nicht signifikant. Das Signifikanzniveau, bei den statistischen Testverfahren der p-Wert, wird üblicherweise zwischen 0,01 und 0,05 definiert. Bei den Testentscheidungen in dieser Arbeit wurde das Signifikanzniveau auf 5\% definiert. Testergebnisse mit einem p-Wert zwischen 0,01 und 0,05 werden als signifikant bezeichnet. Ist der $p$-Wert $<0,01$, so ist die Testentscheidung hochsignifikant.

Zur Berechnung der Daten wurden folgende Testverfahren in dieser Arbeit angewendet (Tab.9): 


\begin{tabular}{|l|l|l|}
\hline Test & $\begin{array}{l}\text { Anzahl und Art der } \\
\text { Stichproben }\end{array}$ & Hypothese \\
\hline $\begin{array}{l}\text { U-Test von Mann, } \\
\text { Whitney und Wilcoxon }\end{array}$ & $\begin{array}{l}\text { Zwei unverbundene } \\
\text { Stichproben bei nicht } \\
\text { normalverteilten Daten }\end{array}$ & $\begin{array}{l}\text { Gleichheit der Mediane in } \\
\text { den Gruppen }\end{array}$ \\
\hline Kruskal-Wallis-Test & $\begin{array}{l}\text { Vergleich von mehr als } \\
\text { zwei unverbundenen } \\
\text { Stichproben bei nicht } \\
\text { normalverteilten Daten }\end{array}$ & $\begin{array}{l}\text { Gleichheit der Mediane in } \\
\text { den }\end{array}$ \\
\hline
\end{tabular}

Tab.9: Verwendete Testverfahren

Die Ergebnisse wurden aus den gültigen vorhandenen Daten berechnet. 


\section{Ergebnisse}

\subsection{Stanzen und Prostatapräparate}

\subsubsection{Anzahl der Stanzen}

Die Daten der präoperativen Stanzenentnahmen lagen in 123 Fällen $(86,7 \%)$ vor. Bei 6 Patienten (4,2\%) wurde im Rahmen einer transurethralen Resektion der Prostata (TUR-P) ein ,incidental carcinoma“ (pT1b) festgestellt.

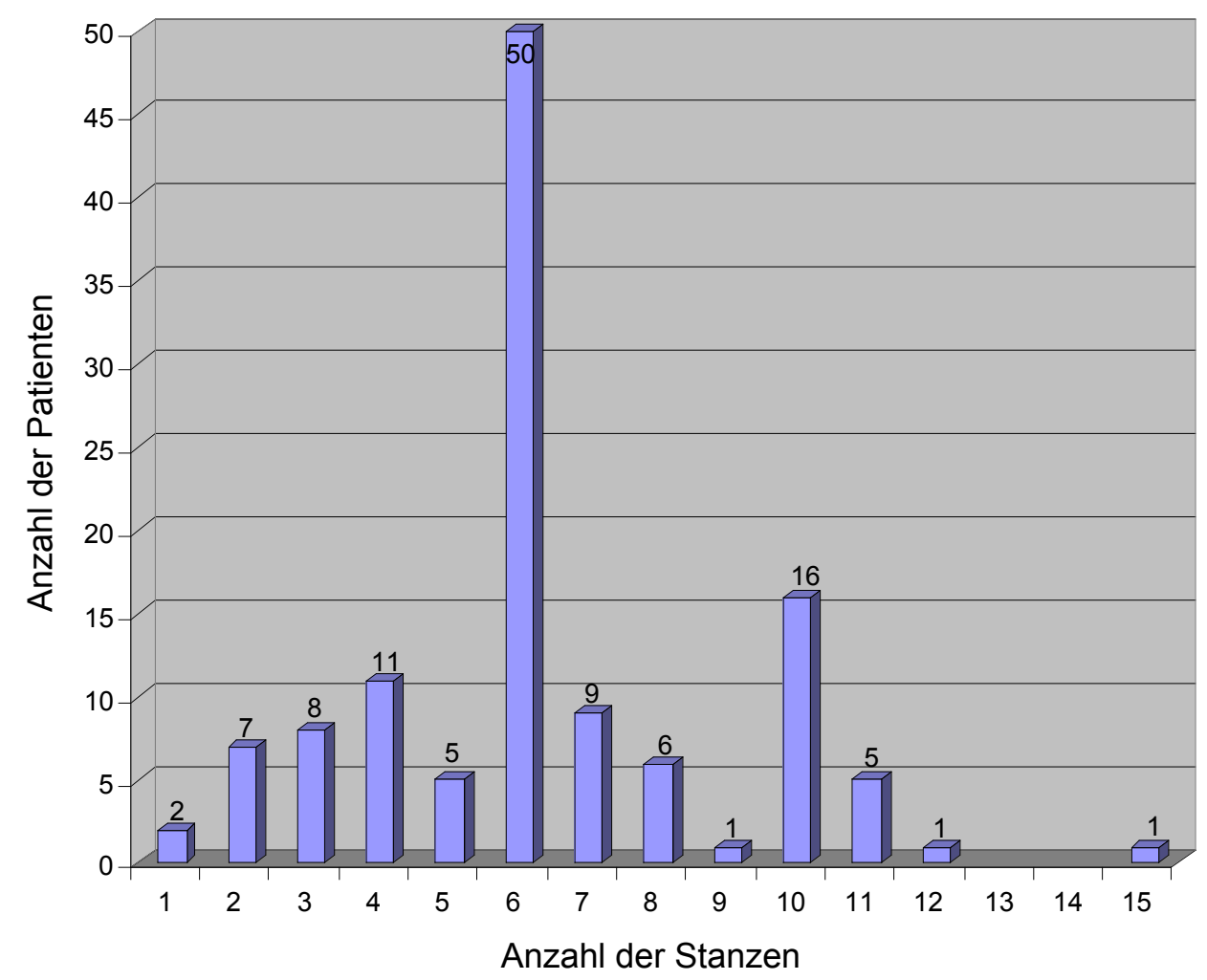

Abb.2: Verteilung und Anzahl der Biopsien (außer einem Patienten mit 27 Biopsien)

In 82 Fällen (66,7\%) wurden zwischen sechs und zehn Stanzen entnommen, wobei die Anzahl der Patienten, bei denen 6 Proben entnommen wurden mit 50 $(40,7 \%)$ am stärksten vertreten ist. Der zweite Peak bei 10 entnommenen Proben mit 16 Patienten(13\%) ergibt sich daraus, dass gegen Ende 2002 die empfohlene Entnahme von 6 auf 10 Gewebszylinder hochgesetzt wurde. 1 bis 5 Stanzzylinder 
wurden bei 33 Patienten (26,8\%) histologisch untersucht, elf oder mehr Gewebeproben wurden in 8 Fällen (6,5\%) biopsiert, wobei im extremsten Fall 27 Stanzen entnommen wurden (Abb.2)

\subsubsection{Histologische Beurteilung}

Die Beurteilung der Biopsien hinsichtlich des Nachweises eines Karzinoms lag bei allen 140 Patienten vor. Bei allen Patienten wurde vor der Operation ein Karzinom histologisch nachgewiesen. Die histologische Beurteilung des Prostatapräparates nach der Operation lag ebenfalls bei allen Patienten vor. Bei 3 Patienten $(2,1 \%)$ konnte im Präparat durch den Pathologen kein Karzinom festgestellt werden.

Die Beurteilung der Stanzen hinsichtlich des Seitenlappenbefalls war in 119 Fällen $(85 \%)$ zu ermitteln.

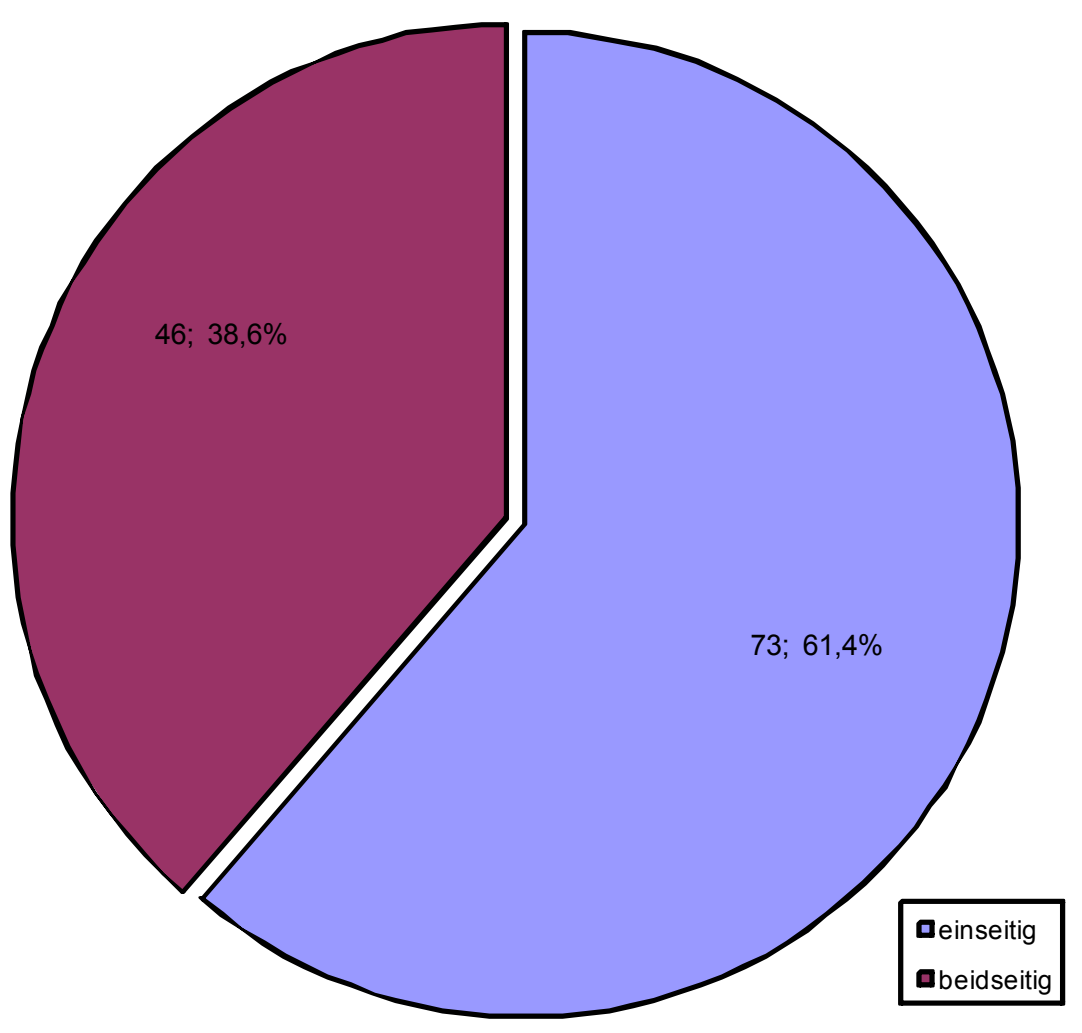

Abb.3: Aufteilung der präoperativ entnommenen Stanzbiopsien nach Seitenlappenbefall 
Bei der histologischen Untersuchung der entnommenen Stanzen fanden die Pathologen in 73 Fällen $(61,4 \%)$ ein Karzinom in den Gewebszylindern eines Prostatalappens. Der Karzinombefall wurde als einseitig eingestuft. Bei 46 Patienten $(38,6 \%)$ wurden die pathologischen Veränderungen in den Proben aus beiden Prostatalappen festgestellt (Abb. 3)

Die histologische Kontrolle der operativ entnommenen Prostatapräparate im Bezug auf die Seitenverteilung des Karzinoms lag in 130 Fällen (92,9\%) vor.

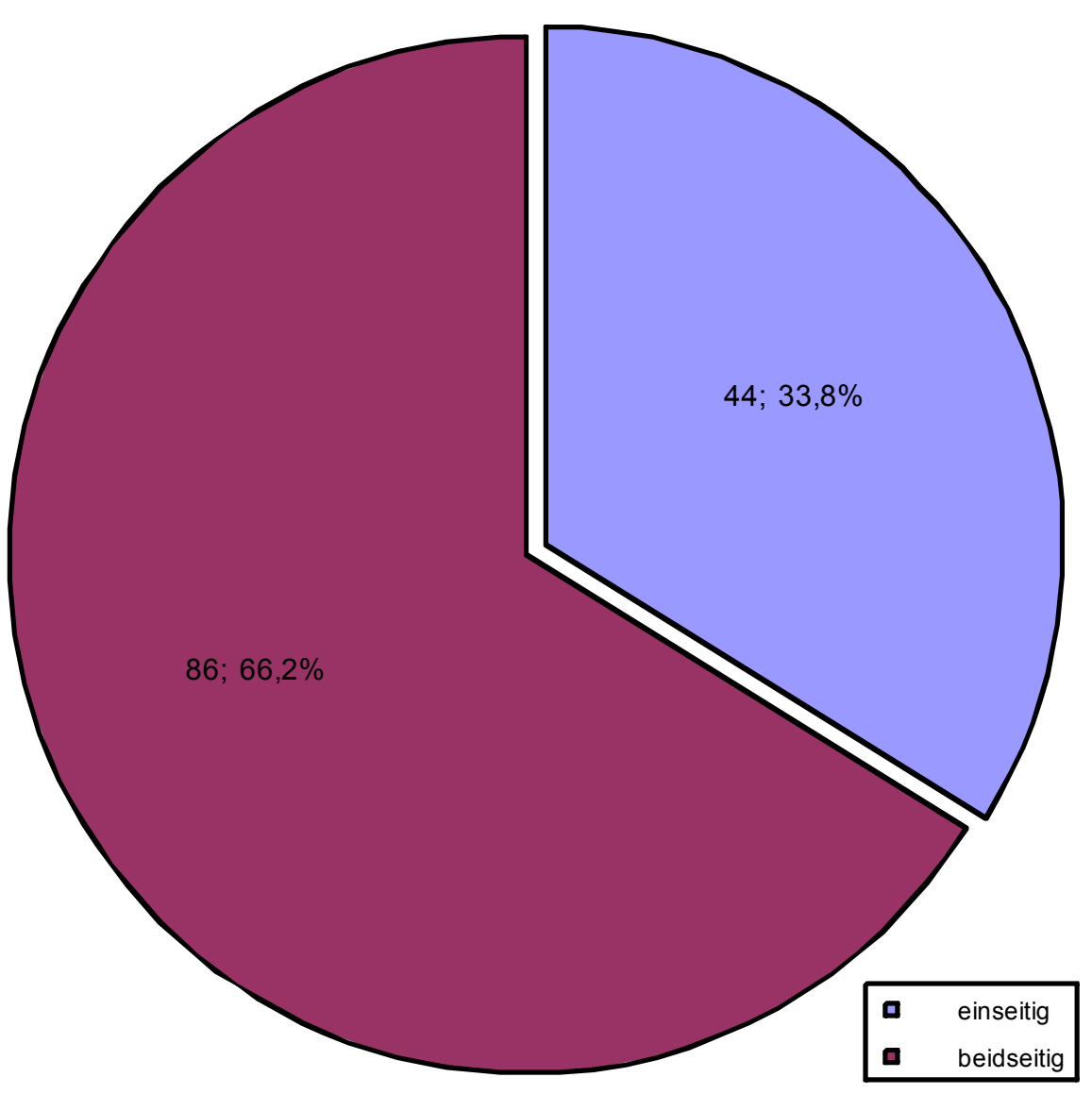

Abb.4: Aufteilung der postoperativen Prostatapräparate nach Seitenlappenbefall

Bei 44 Präparaten (33,8\%) wurde ein einseitiger Befall festgestellt. Bei der Analyse der restlichen 86 Prostatae (66,2\%) fand der Pathologe in beiden Seitenlappen die Veränderungen (Abb.4). 


\subsubsection{Sensitivität und Spezifität der Stanzbiopsien}

Die histopathologischen Befunde aus den Voruntersuchungen wurden mit den Befunden der operativ entnommenen Präparate verglichen. Somit können Rückschlüsse auf die Sensitivität und Spezifität der präoperativen Diagnostik hinsichtlich der Erkennung des ein- bzw. beidseitigen Befalls gezogen werden.

Abbildung 5 zeigt die Ergebnisse der präoperative Stanzbiopsie für alle Patienten, bei denen das Prostatapräparat in der postoperativen histopathologischen Untersuchung ein einseitiges Karzinom aufwies, das heißt nur ein Seitenlappen befallen war. In 45 Fällen waren die entsprechenden Daten zu ermitteln.

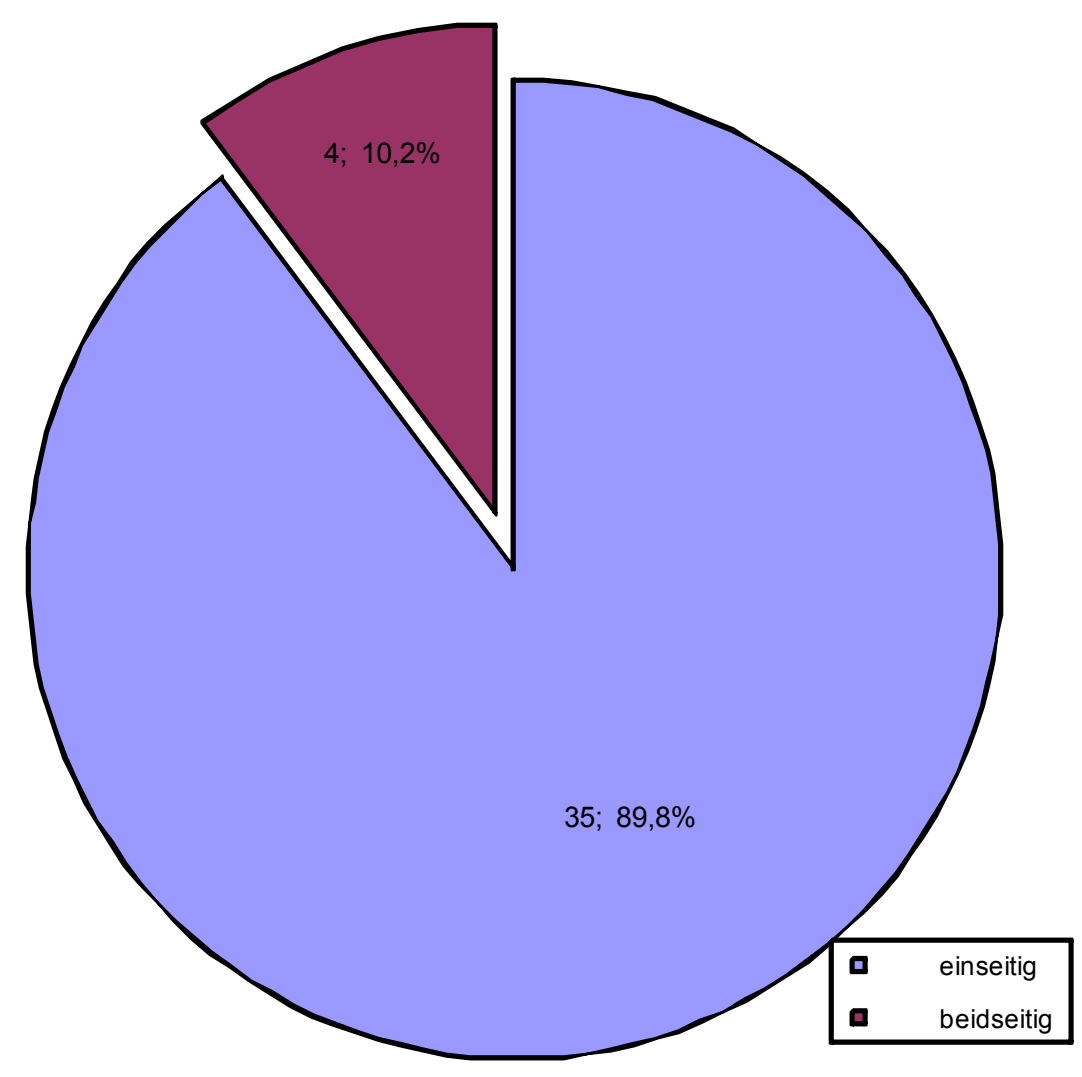

Abb.5: Aufteilung der Stanzbiopsien nach Befall der Seitenlappen aller Patienten, bei denen postoperativ ein einseitiges Karzinom gesichert wurde. 
Bei 35 Patienten wurde das einseitige Karzinom der Prostata durch die Stanzenentnahme richtig erkannt (89,8\%). Bei 4 Patienten (10,2\%) wurde in der präoperativen Diagnostik ein Karzinom in beiden Seitenlappen diagnostiziert, bei der histopathologischen Untersuchung nach der Operation wurde dieses aber nur in einem Seitenlappen festgestellt (Abb.5).

Abbildung 6 zeigt die Ergebnisse der präoperativen Stanzbiopsie bei der Patientengruppe, bei der das Prostatapräparat in der postoperativen histopathologischen Untersuchung beidseits ein Karzinom aufwies, das heißt beide Seitenlappen befallen waren. Die entsprechenden Daten konnten in 73 Fällen ermittelt werden.

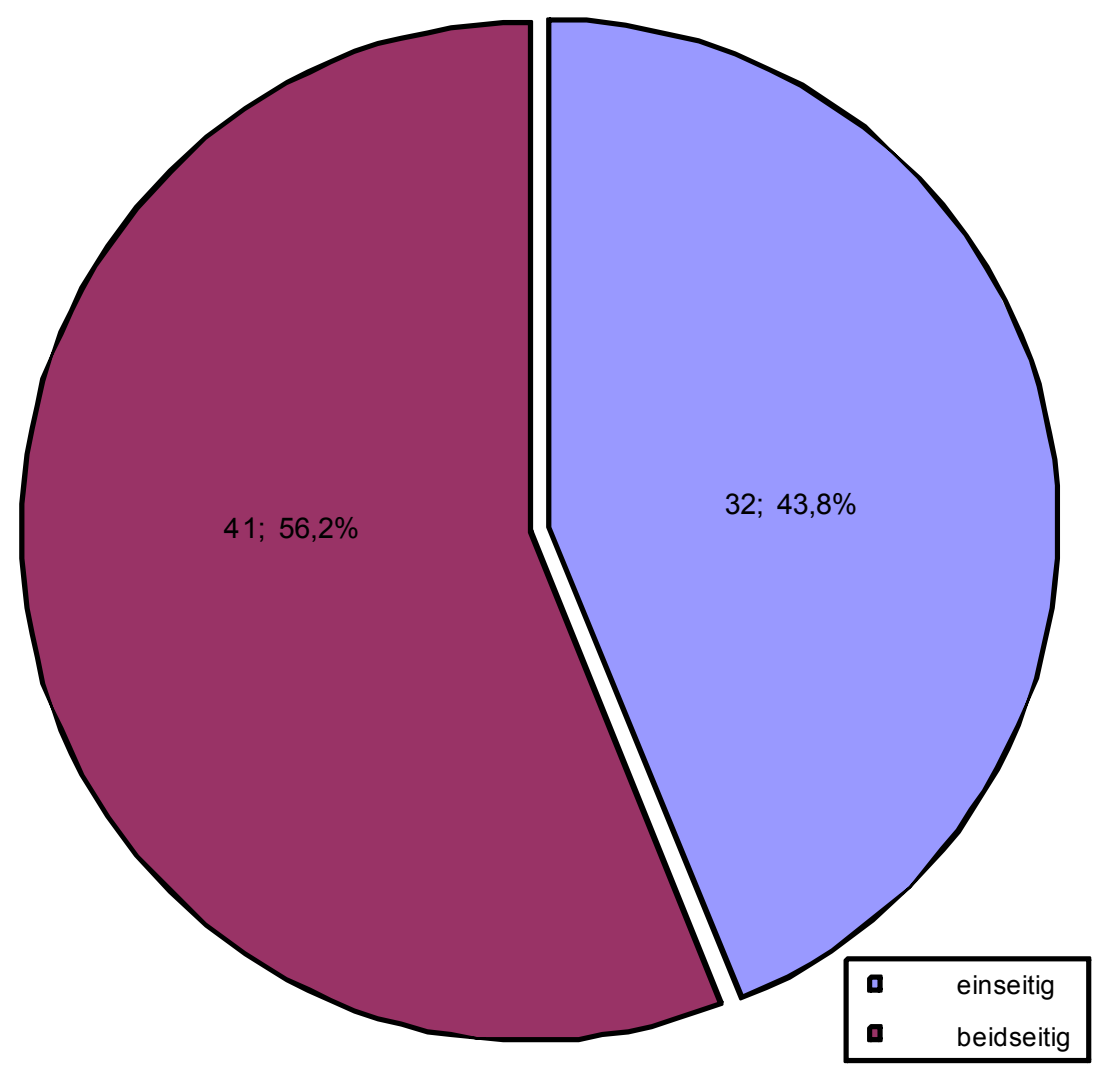

Abb.6: Aufteilung der Stanzbiopsien nach Befall der Seitenlappen aller Patienten, bei denen postoperativ ein beidseitiges Karzinom gesichert wurde

Bei 41 Patienten wurde der beidseitige Befall des Drüsengewebes durch die Stanzbiopsie richtig erkannt (56,2\%). Demgegenüber konnte bei 32 Patienten 
$(43,8 \%)$ das Karzinom im zweiten Seitenlappen durch die Stanzen nicht gesichert werden (Abb.6).

Somit errechnet sich hinsichtlich der Erkennung eines bilateralen Karzinombefalls der Prostata durch Stanzbiopsien eine Spezifität von 90,7\% und eine Sensitivität von $69,5 \%$.

\subsubsection{Vergleich von 6 entnommenen gegenüber $>=10$ entnommenen Stanz- zylindern}

Der Vergleich der Patientengruppe, bei der exakt 6 Stanzzylinder entnommen wurden mit derjenigen, bei welcher 10 oder mehr Stanzen entnommen wurden, ist in folgender Grafik veranschaulicht (Abb.7). Bei beiden Patientengruppen lag sowohl ein prä- als auch ein postoperatives histologisches Ergebnis im Bezug auf uni- und bilateralen Tumorbefall der Prostata vor.

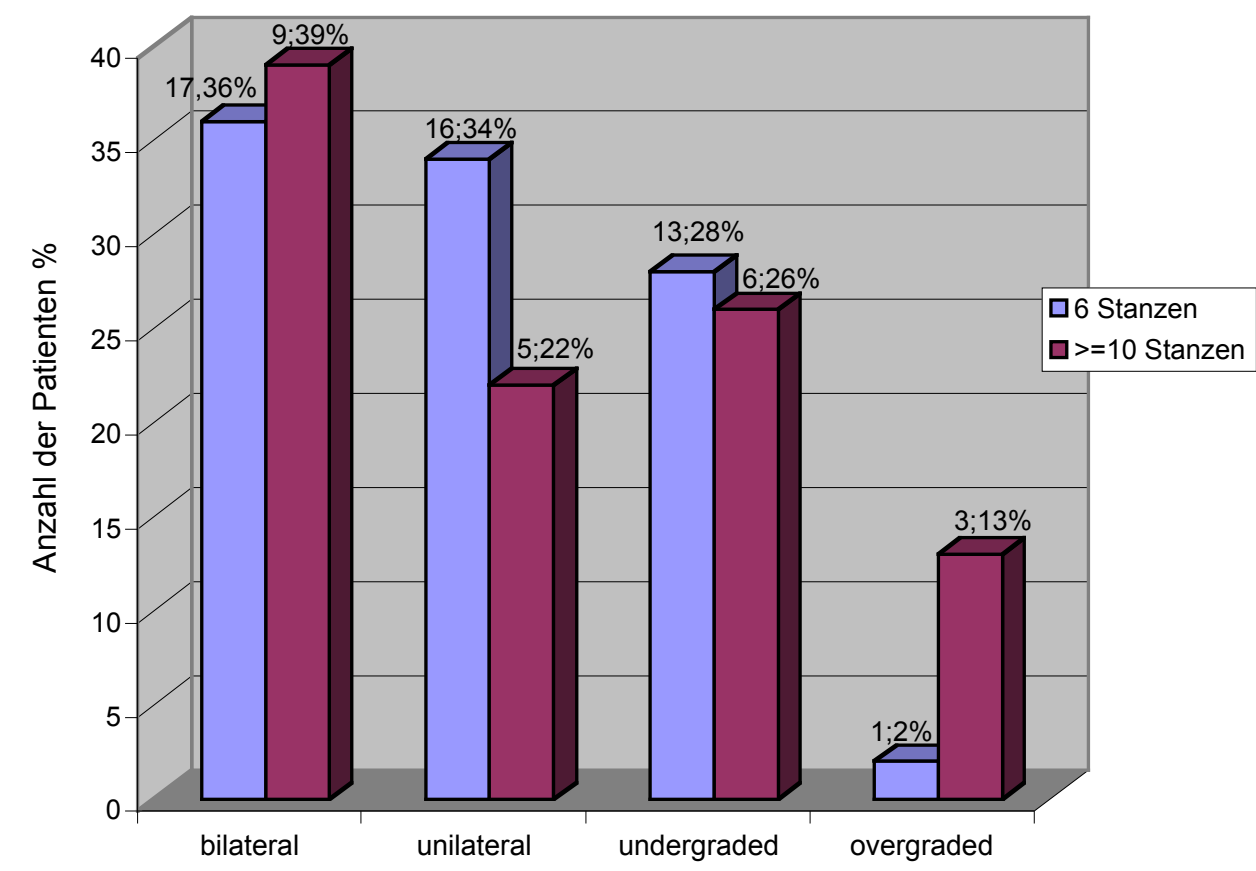

Abb.7: Vergleich von $6 \mathrm{zu}>=10$ entnommenen Stanzen im Bezug auf die Übereinstimmung des prä- zu dem postoperativen histologischen Ergebnis ( uni- vs. bilateraler Befall)

Die Voraussetzungen für die Patientengruppe, bei der 6 Stanzen entnommen wurden, erfüllten $n=47$ unserer Patienten. Auf $n=23$ Patienten traf dies bei 10 oder 
mehr entnommenen Stanzen zu. Ein sowohl prä- als auch postoperativ bilateraler Tumorbefall wurde bei 36 bzw. 39\% (n=17 bzw. 9) der Patienten eruiert. Ein unilateraler Befall fand sich bei 34 bzw. 22\% ( $n=16$ bzw. 5) der Patienten. Ein präoperativ als unilateral eingestufter Tumorbefall, der postoperativ beide Seitenlappen befallen hatte, zeigte sich bei 28 bzw. $26 \%$ (n=13 bzw. 6) der Patienten. Bei 2 bzw. 13\% (n=1 bzw. 3) der Patienten wurde in den Stanzen aus beiden Prostataseitenlappen Tumorbefall festgestellt, wobei postoperativ dies nur noch für einen Seitenlappen zutraf.

Es ist statistisch kein signifikanter Unterschied $(\mathrm{p}=0,45)$ feststellbar.

\subsubsection{Sensitivität der Stanzbiopsien in Abhängigkeit von der Anzahl der Stanzen}

Das Patientenkollektiv wurde nach der Anzahl der Stanzen in drei Gruppen eingeteilt:

1.Gruppe $=1-5$ Stanzen, 2.Gruppe $=6-10$ Stanzen, 3.Gruppe $=>=11$ Stanzen

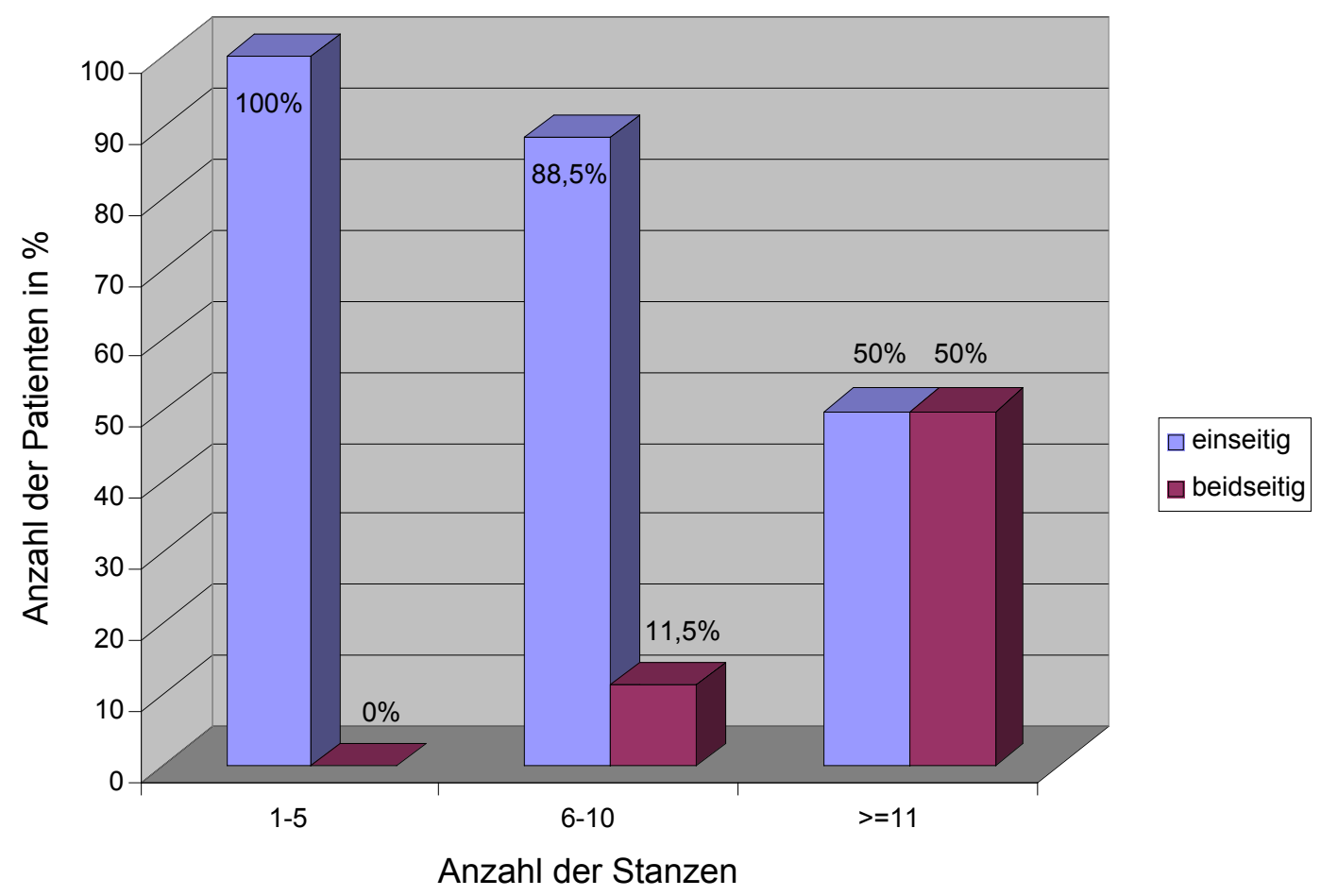

Abb.8: Sensitivität der Stanzen in Bezug auf den Seitenlappenbefall in Abhängigkeit von der Anzahl der Stanzen bei einseitigem Tumorbefall der Prostata 
Bei postoperativ einseitigem Tumor in der Gruppe 1(n=11) wurde der Lappenbefall $\mathrm{zu}$ 100\% richtig erkannt, in Gruppe $2(\mathrm{n}=26)$ noch $\mathrm{zu} 88,5 \%$ und in der 3. Gruppe (n=2) nur noch zu 50\% (Abb.8).

Hatte der Tumor beide Seitenlappen befallen, so wurde dies in der 1.Gruppe $(n=17)$ in 53\% der Fälle richtig erkannt, in Gruppe $2(n=49)$ in 56\% der Fälle und in Gruppe $3(n=4)$ bei $75 \%$ der Biopsien (Abb.9).

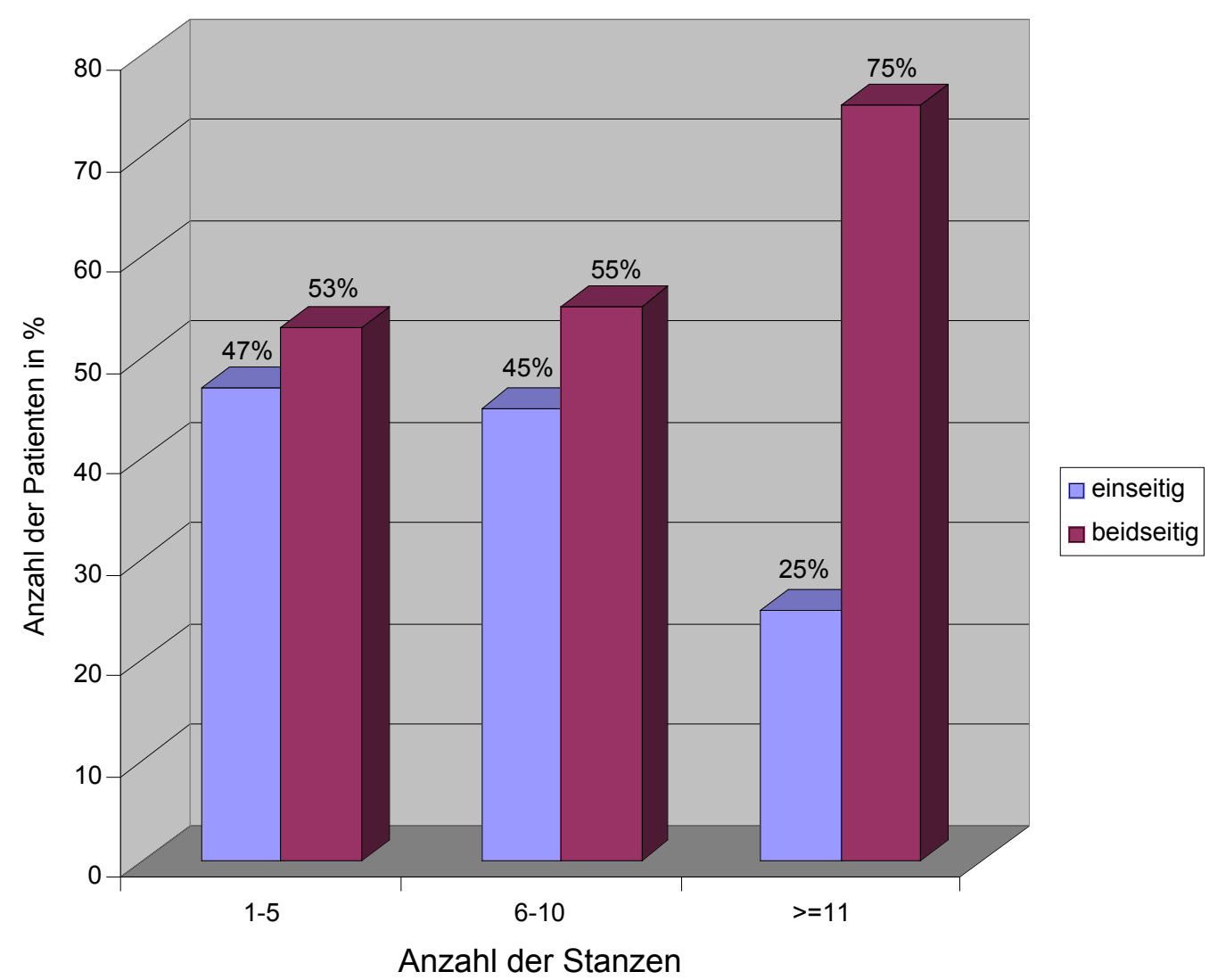

Abb.9: Sensitivität der Stanzen in Bezug auf den Seitenlappenbefall in Abhängigkeit von der Anzahl der Stanzen bei beidseitigem Tumorbefall der Prostata

\subsubsection{Analyse der Daten hinsichtlich des Absetzungsrandes der Prostataprä- parate}

Die Prostatapräparate wurden vom Pathologen nach positivem (R1) und negativem (R0) Absetzungsrand beurteilt. Diese Beurteilungen lagen in 137 Fällen $(97,9 \%)$ vor. 


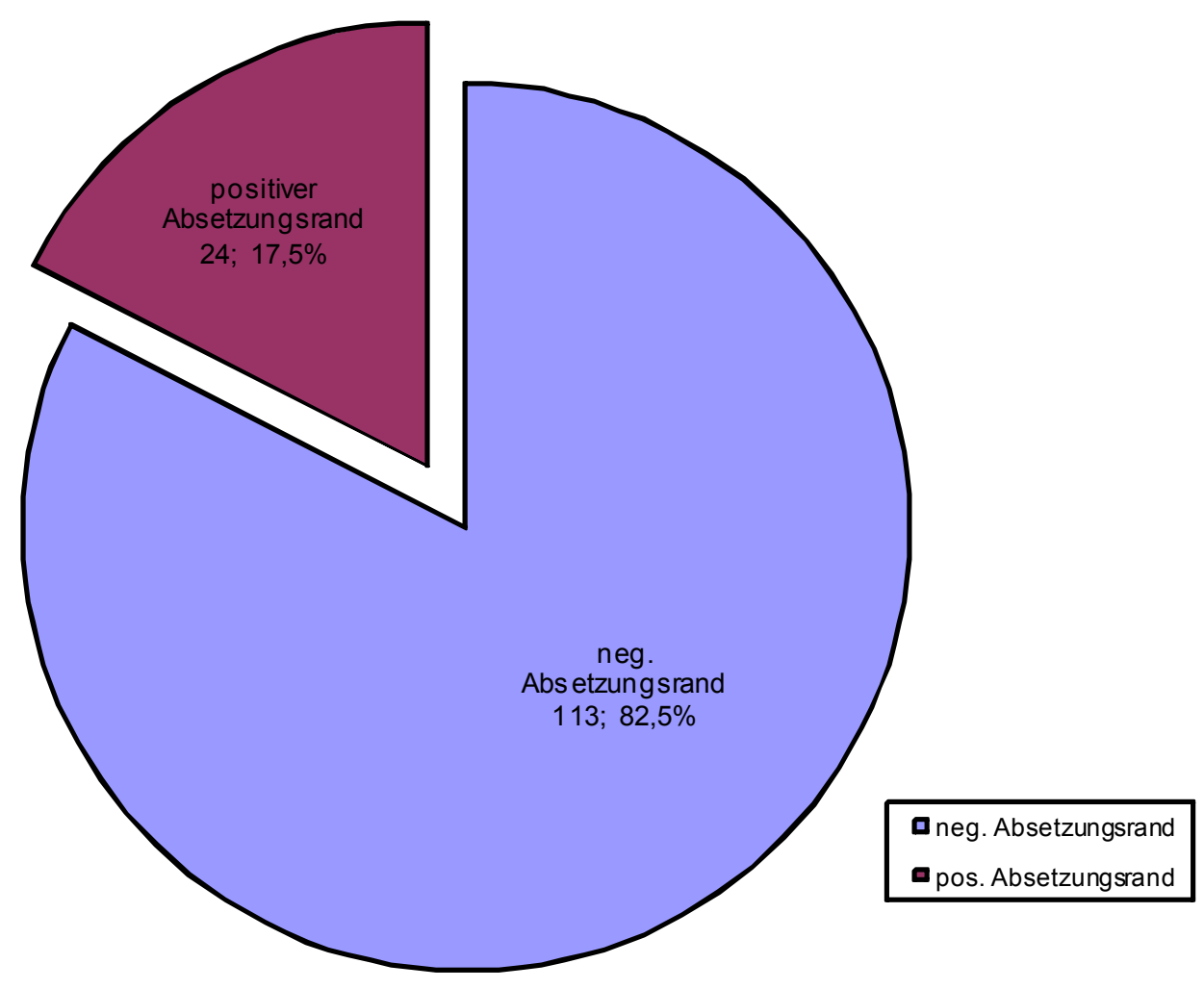

Abb.10: Anzahl der negativen und positiven Absetzungsränder

Bei 113 Prostatektomien (82,5\%) konnte das maligne Gewebe vollständig entfernt werden. In 24 Fällen (17,5 \%) haben die Pathologen einen positiven Absetzungsrand festgestellt. Mit hoher Wahrscheinlichkeit sind bei diesen Patienten Reste mit karzinomatös verändertem Gewebe nicht entfernt worden. Der histologische Befund R1 kann aber auch bedeuten, dass der notwendige Sicherheitsabstand bei der Resektion nicht eingehalten, aber alle malignen Zellen entfernt wurden (Abb.10). 


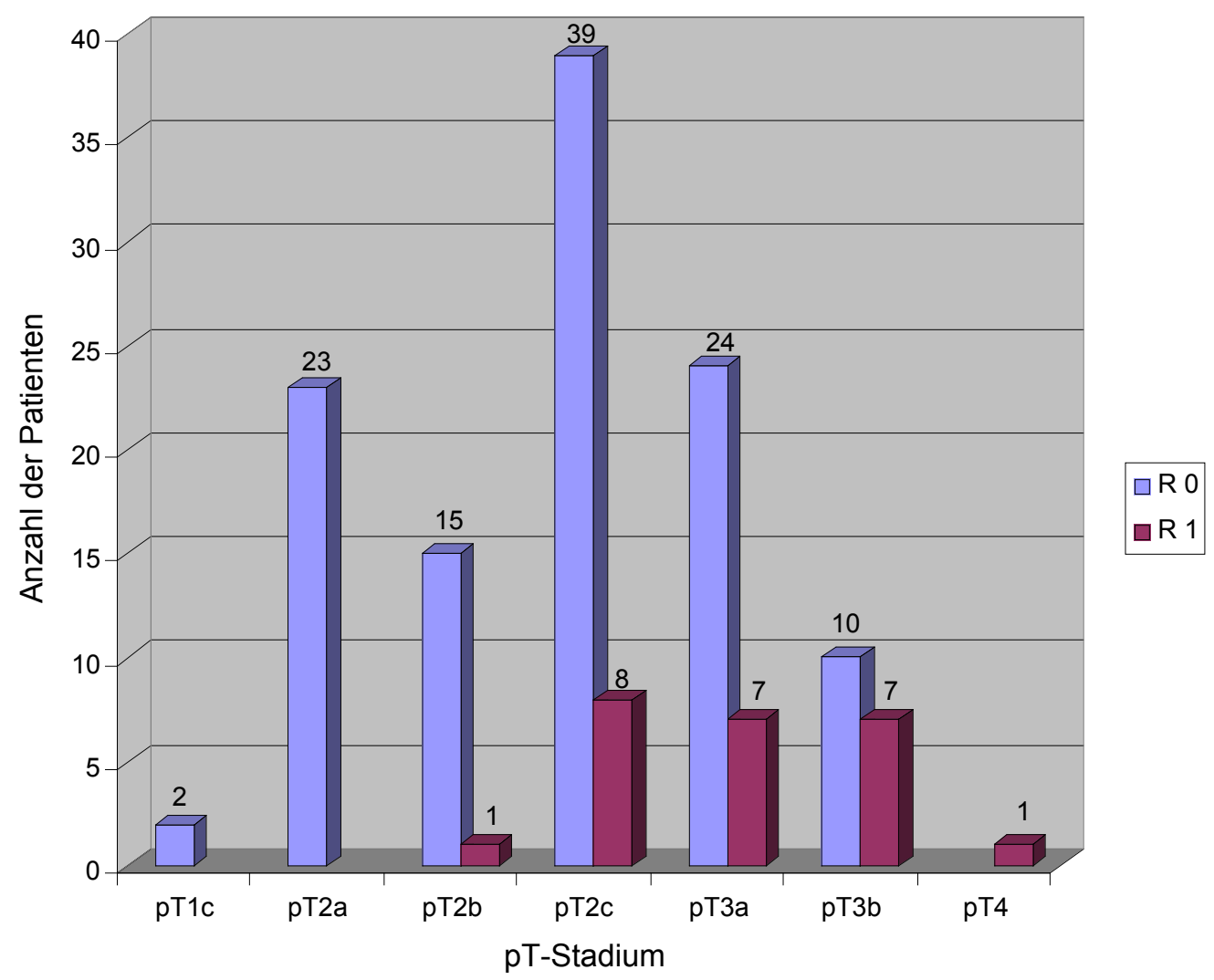

Abb.11: Tumorstadien der Fälle mit positivem Absetzungsrand im Verhältnis zu den R0-Resezierten

Betrachtet man die Tumorstadien der Patienten mit positivem Absetzungsrand, so fällt auf, dass in 9 Fällen der Tumor auf die Prostata begrenzt war. In den restlichen 15 Fällen hatte das Tumorwachstum die Prostatakapsel bereits durchbrochen. Die genauen Tumorstadien dieser R1-resezierten Patienten sind in Abbildung 11 dargestellt.

Die Analyse der prä- zu den postoperativen Histologien im Bezug auf uni- bzw. bilateralen Tumorbefall zeigt im Vergleich der R0- Resezierten ( $\mathrm{n}=96)$ mit den R1-Resezierten ( $\mathrm{n}=20)$ folgendes Ergebnis. 


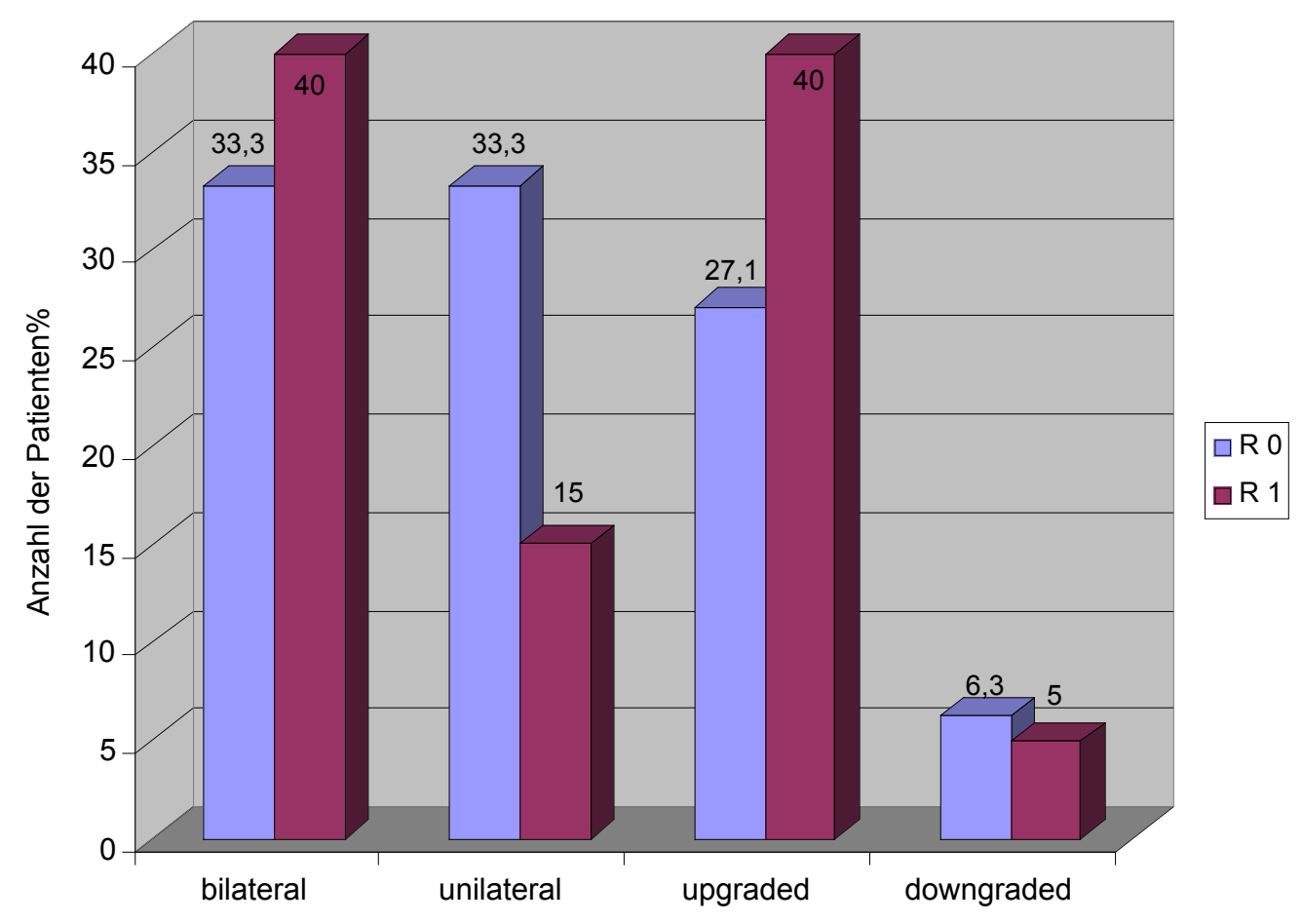

Abb.12: Vergleich von R1- zu R0- Resektion im Bezug auf die Übereinstimmung des prä- zu dem postoperativen histologischen Ergebnis ( uni- vs. bilateraler Befall)

Ein Befall beider Seitenlappen der Prostata sowohl bei der Biopsie als auch im postoperativen Präparat zeigte sich in 33,3 bzw. $40 \%$ der R0- bzw. R1Resezierten. Ein unilateraler Tumorbefall der R0- bzw. R1-Gruppe wurde bei 33,3 bzw. 15\% festgestellt. Ein präoperativ unilateraler Befall, der postoperativ als bilateral eingestuft wurde, fand sich bei den R0-Resezierten in 27,1\% der Fälle, in der Gruppe der R1-Resezierten wurde ein ,upgrading“ bei 40\% registriert. Ein „downgrading“ fand in 6,3 bzw. 5\% der Patienten statt (Abb.12). 


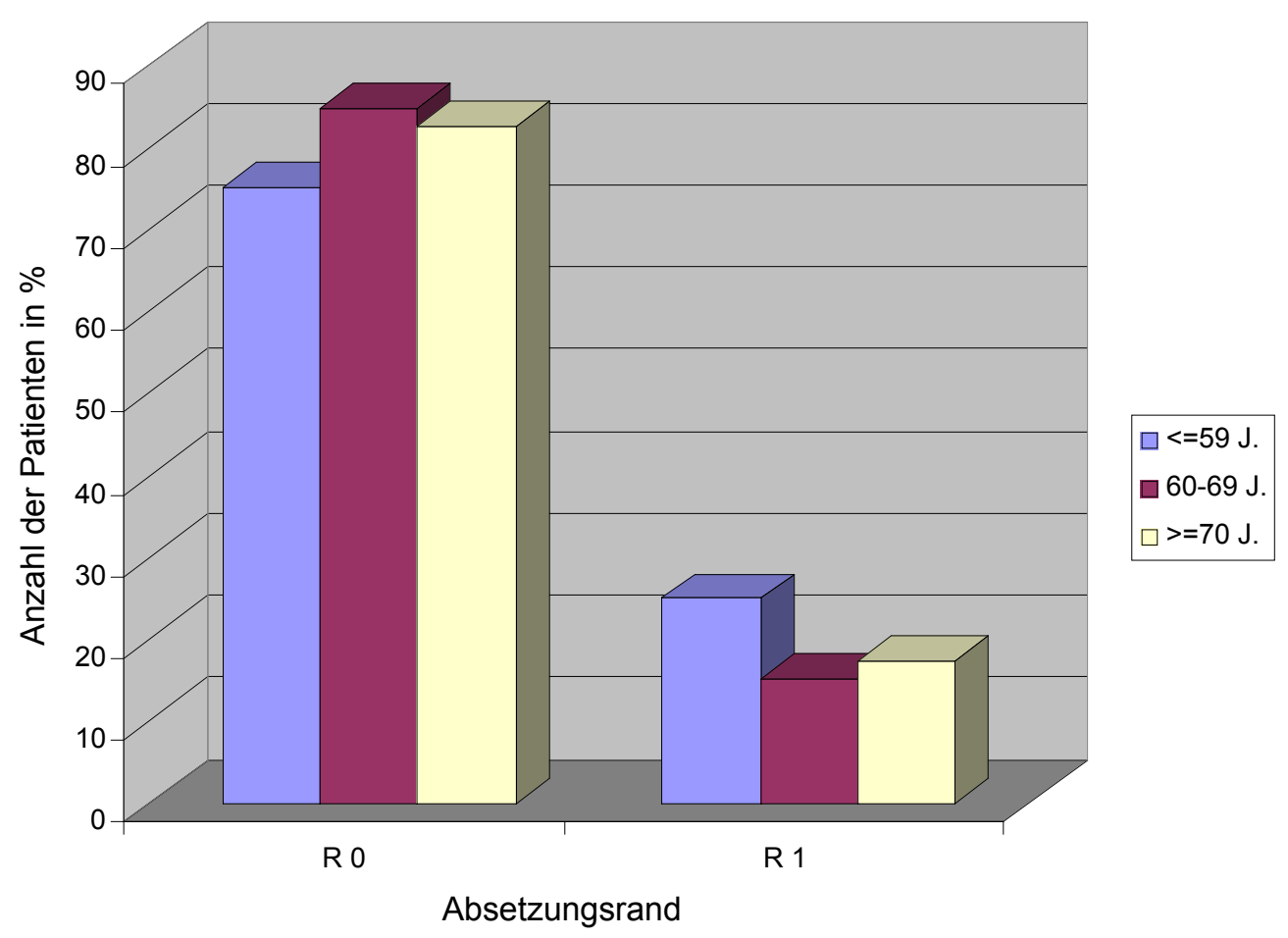

Abb.13: Absetzungsrand in Abhängigkeit vom Lebensalter

Die Analyse der Daten hinsichtlich der Altersgruppen ergab, dass das Lebensalter der Patienten auf den Absetzungsrand keinen Einfluss hat. Statistisch ist kein signifikanter Unterschied zwischen den Gruppen messbar (Kruskal-Wallis-Test mit $\mathrm{p}=0,492)$.

\subsection{Analyse der Daten hinsichtlich der pTNM-Klassifikation}

\subsection{1 pT-Stadium}

In unserem Patientenkollektiv reichen die histologisch gesicherten Tumorstadien von pT2a bis pT4. Die Stadien pT1a, pT1b und pT1c sind postoperativ per Definition nicht vertreten. Das definitive postoperative Tumorstadium ließ sich in 135 Fällen $(96,4 \%)$ ermitteln. In 2 Fällen $(1,4 \%)$ konnte postoperativ in der Prostata kein Karzinom nachgewiesen werden. 


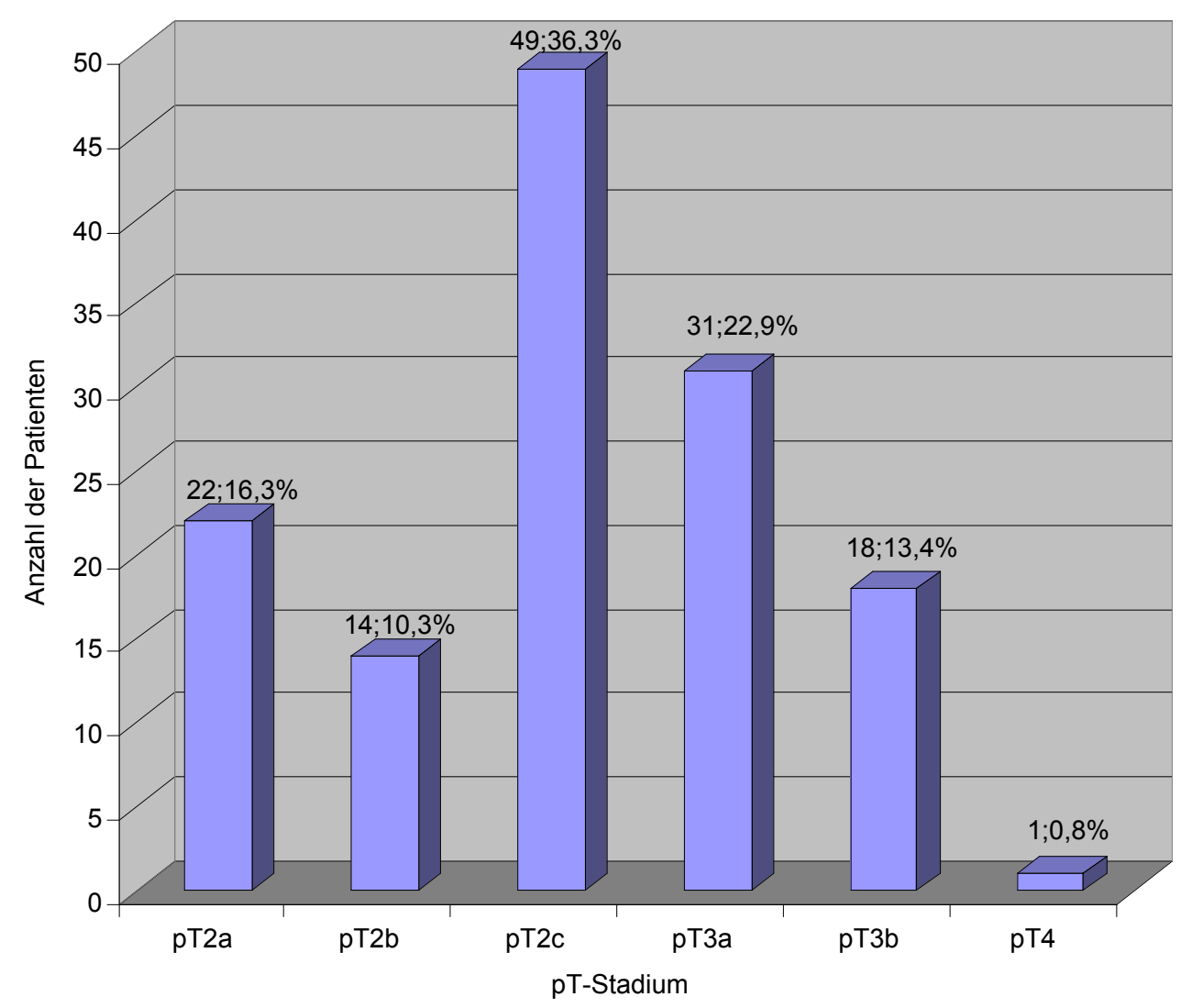

Abb.14: Verteilung der Tumorstadien

Bei einer Mehrheit von 49 Patienten (36,3\%) wurde von den Pathologen ein pT2c-Stadium festgestellt. Es folgen die Stadien pT3a mit 31 Fällen (22,9\%), pT2a mit 22 Fällen (16,3\%), pT3b mit 18 Fällen (13,4\%), pT2b mit 14 Fällen $(10,3 \%)$ und schließlich wurde bei nur einem Patienten $(0,8 \%)$ das Stadium pT4 festgestellt (Abb.14).

Die Häufigkeiten der Tumore, die auf die Prostata begrenzt waren $(<=p T 2 c)$, beziehungsweise das Organ überschritten hatten $(>=\mathrm{pT} 3 \mathrm{a})$ sind in Tabelle 10 dargestellt. 


\begin{tabular}{|l|c|c|}
\hline & Anzahl & $\%$ \\
\hline$<=$ pT2c & 85 & 63 \\
organbegrenzt & & \\
\hline$>=$ pT3a & 50 & 37 \\
organüberschreitend & & \\
\hline
\end{tabular}

Tab.10: Verteilung der Tumorstadien: organbegrenztes und organüberschreitendes Prostatakarzinom

Der Anteil der auf das Organ begrenzten Tumore zeigt eine Häufigkeit von 63\% auf. Entsprechend ist der Anteil der organüberschreitenden Prostatakarzinome $37 \%$ (Tab.10).

\subsubsection{1 p-T Stadium in Abhängigkeit vom Alter}

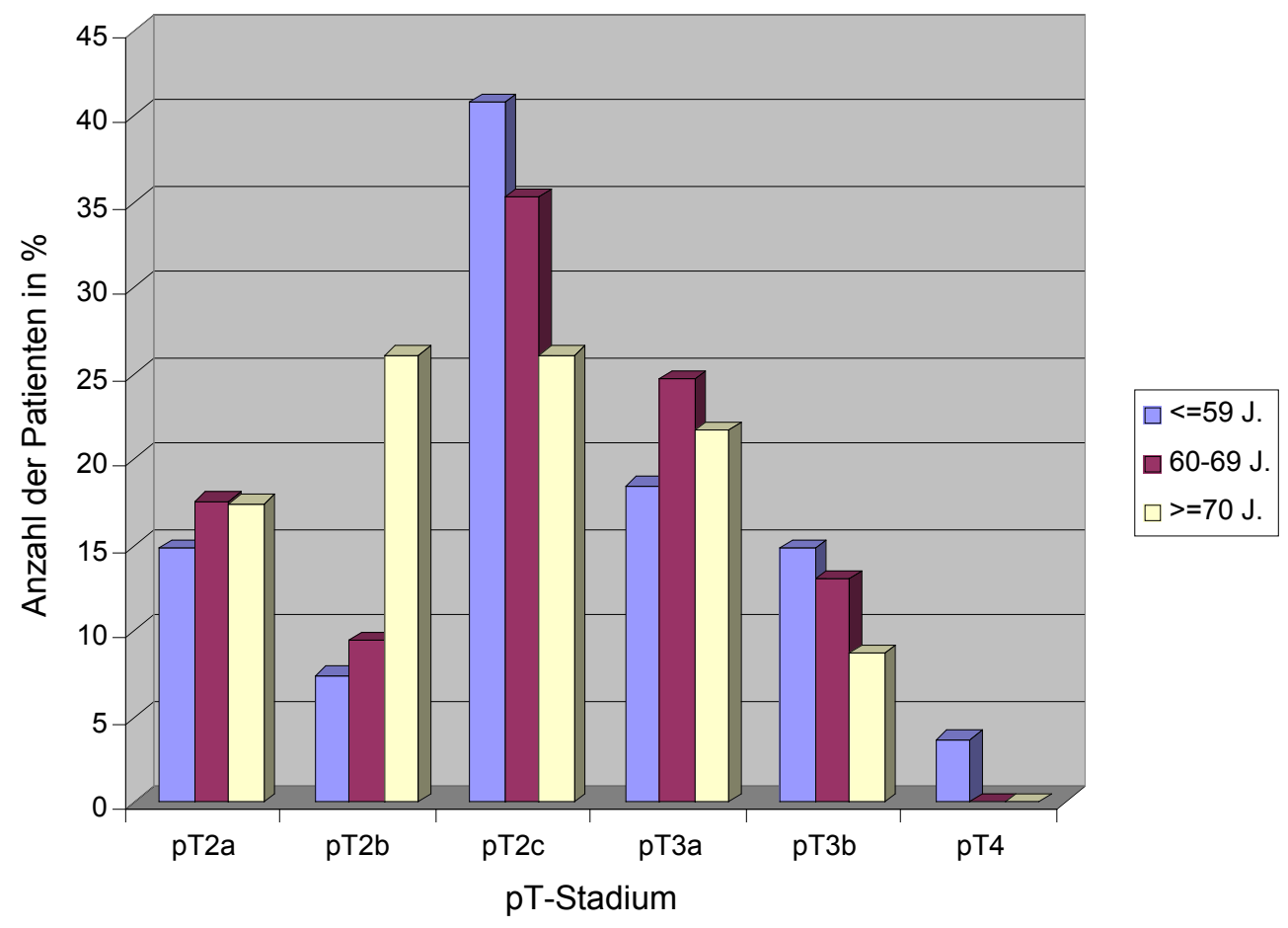

Abb.15: pT-Stadium in Abhängigkeit vom Lebensalter 
Betrachtet man das pT-Stadium in Abhängigkeit vom Lebensalter der Patienten, so zeigt sich kein statistisch signifikanter Unterschied zwischen den Altersgruppen (Kruskal-Wallis-Test mit $\mathrm{p}=0,609)$.

\subsection{2 p-N-Stadium}

Die histologische Beurteilung der Lymphknoten lag in 136 Fällen (97,2\%) vor. Im Durchschnitt wurden 13,2 Lymphknoten entnommen, bei einem Minimum von 2 und einem Maximum von 30 Lymphknoten.

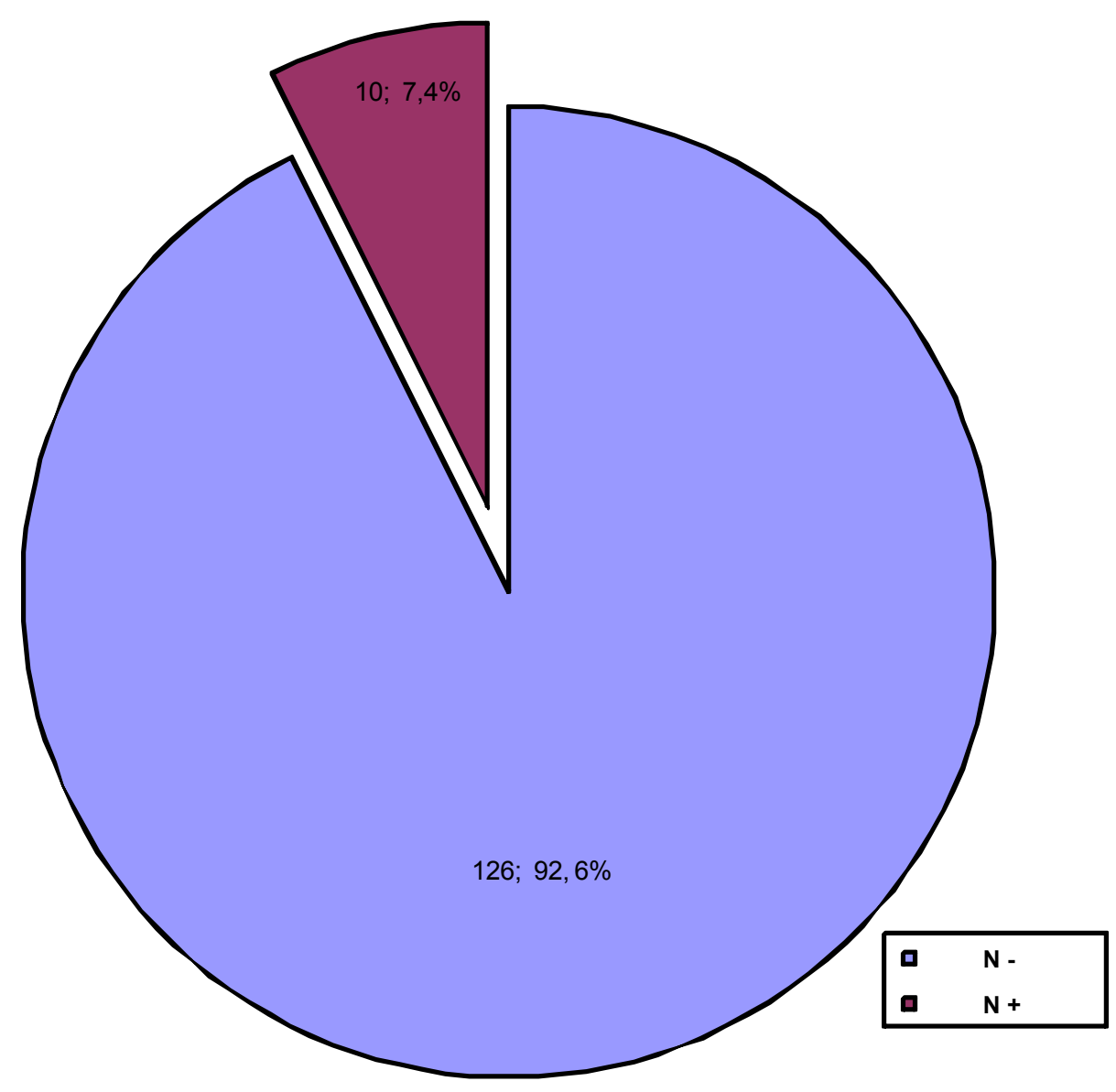

Abb.16: Aufteilung der Fälle nach Lymphknotenmetastasen

Bei der histologischen Beurteilung der intraoperativ entnommenen Lymphknoten konnten bei 126 Patienten (92,6\%) keine Metastasen (N-) festgestellt werden. In 10 Fällen (7,4\%) wurden Lymphknotenmetastasen $(\mathrm{N}+)$ nachgewiesen (Abb.16). 
Die Tumorstadien der Patienten mit Lymphknotenmetastasen sind wie folgt verteilt.

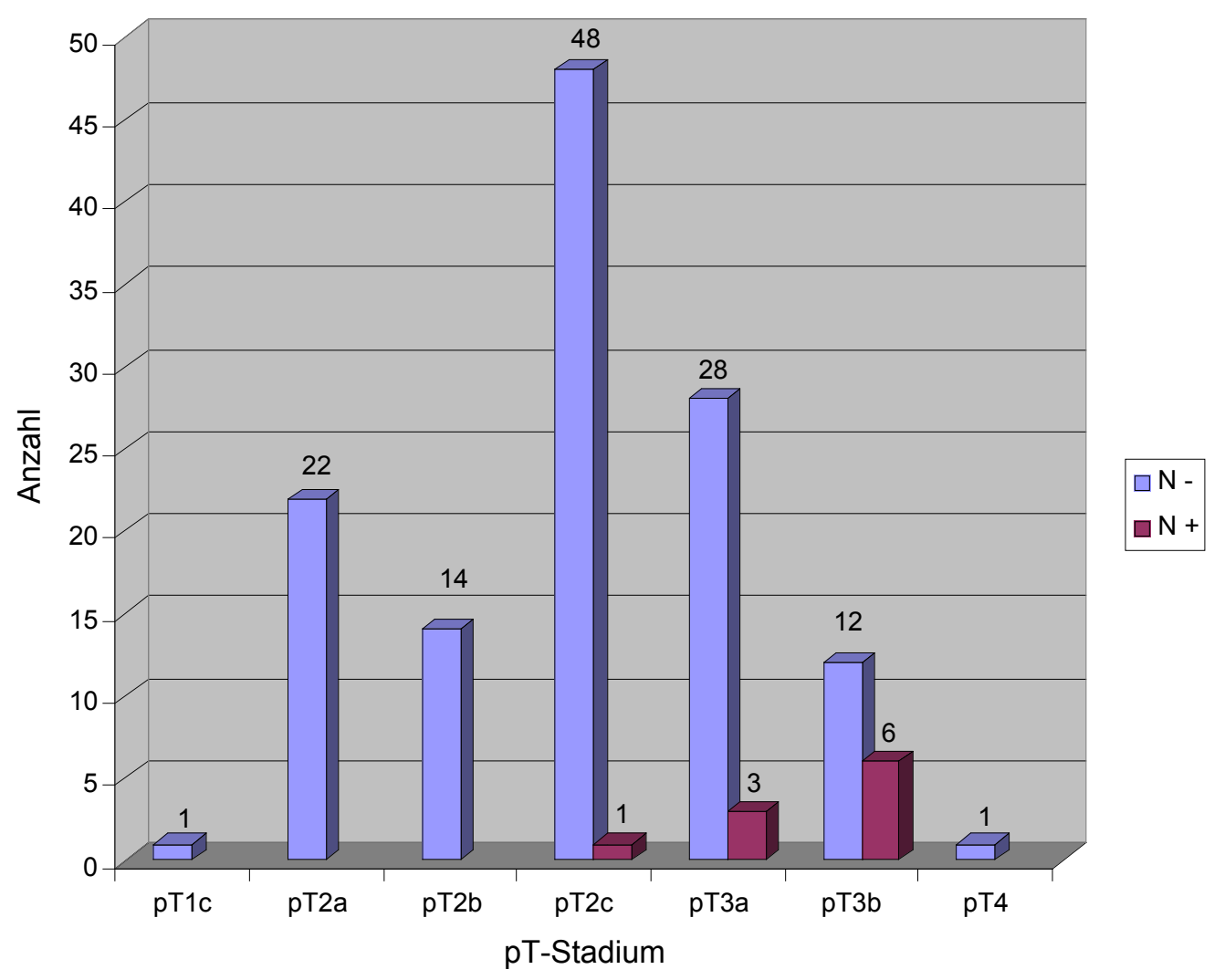

Abb.17: Tumorstadien der Fälle mit positivem Lymphknotenstatus im Verhältnis zu den Fällen mit negativem Lymphknotenstatus

6 Patienten (60\%) weisen ein p-T-Stadium 3b auf, 3 Lymphknotenpositive (30\%) ein pT3a und in einem Fall (10\%) ließ sich ein pT-Stadium 2c nachweisen (Abb.17).

Der Vergleich der Tumorstadien der beiden Gruppen $(\mathrm{N}-$ und $\mathrm{N}+$ ) zeigt einen hochsignifikanten Unterschied (Mann-Whitney U Test mit p=0,0004). Die Fälle mit positivem Lymphknotenstatus weisen im Median ein wesentlich höheres Tumorstadium auf. 


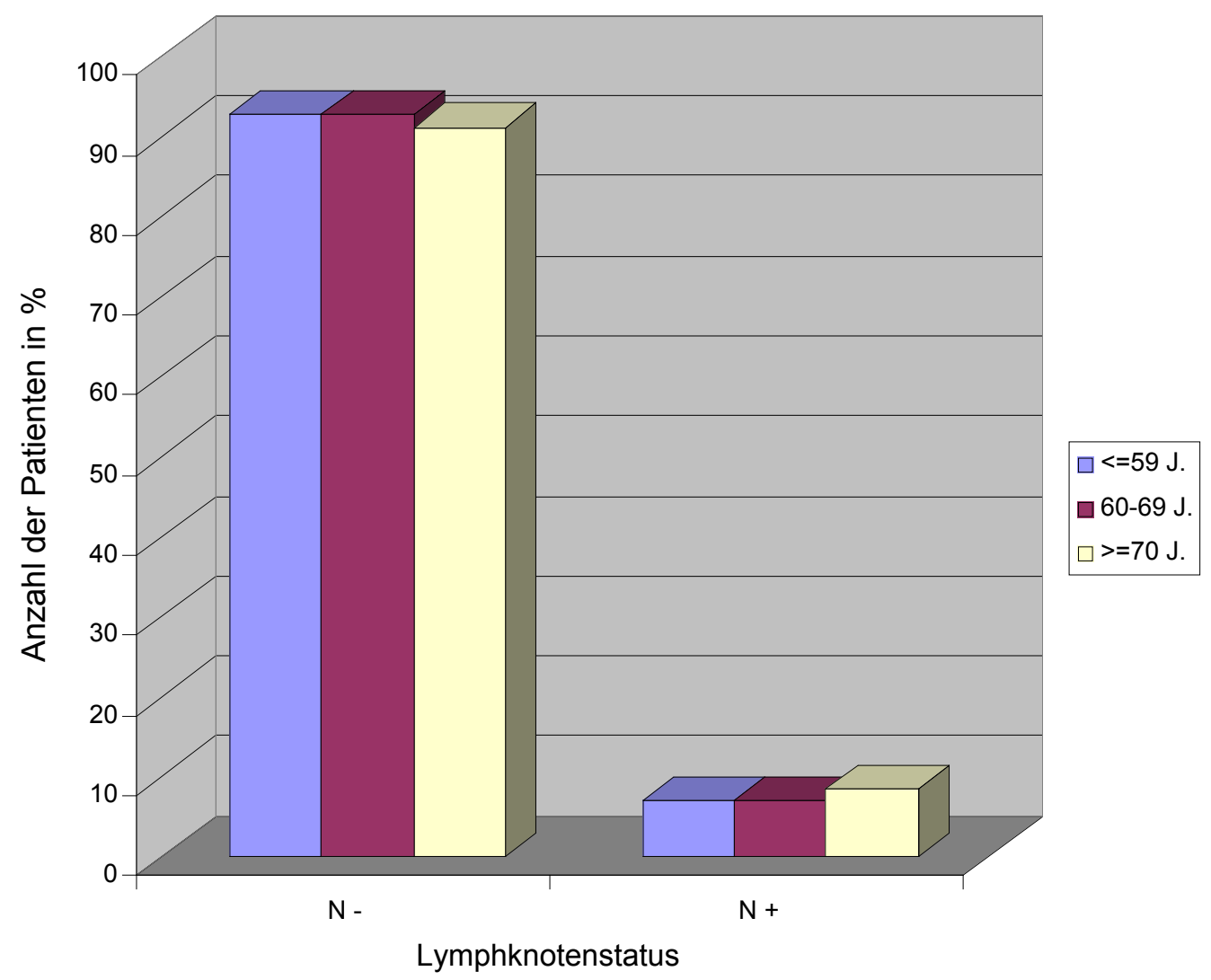

Abb.18: Lymphknotenstatus in Abhängigkeit vom Lebensalter

Betrachtet man den Lymphknotenstatus in Abhängigkeit vom Lebensalter der Patienten, so zeigt sich kein statistisch signifikanter Unterschied zwischen den Altersgruppen (Kruskal-Wallis-Test mit $\mathrm{p}=0,964)$.

\subsection{3 p-M-Stadium}

Metastasen sind bei keinem der operierten 140 Patienten bekannt.

\subsection{Analyse der Daten hinsichtlich des Gradings}

Bei 130 Patienten (92,8\%) liegt das präoperative Grading vor. Bei der histopathologischen Beurteilung wurden 27 Gewebeproben (20,7\%) mit Grad 1 klassifiziert, $87(66,9 \%)$ mit Grad 2 und 16 Biopsien (12,4\%) zeigten einen Grad 3.

Postoperativ wurden 137 (97,8\%) Tumore nach Mostofi und der WHO eingeteilt. In 10 Fällen (7,3\%) zeigt sich ein Grad 1, in 88 (64,2\%) ein Grad 2 und in 39 (28,5\%) ein Grad 3 (Tab.11). 


\begin{tabular}{|l|c|c|c|c|}
\hline Grading & Grad 1 & Grad 2 & Grad 3 & Gesamt \\
\hline präoperativ & $27(20,7 \%)$ & $87(66,9 \%)$ & $16(12,4 \%)$ & $130(100 \%)$ \\
\hline postoperativ & $10(7,3 \%)$ & $88(64,2 \%)$ & $39(28,5 \%)$ & $137(100 \%)$ \\
\hline
\end{tabular}

Tab.11: Verteilung der prä- und postoperativen Grading-Klassifikation

Vergleicht man die präoperativ ermittelten Grade mit der definitiven postoperativen Klassifikation, so zeigt es sich, dass in 81 Fällen $(63,3 \%)$ das präoperative Grading mit dem postoperativen Ergebnis übereinstimmt. 40 Tumore (31,2\%) wurden präoperativ zu niedrig und 7 Tumore $(5,5 \%) \mathrm{zu}$ hoch klassifiziert (Abb.19).

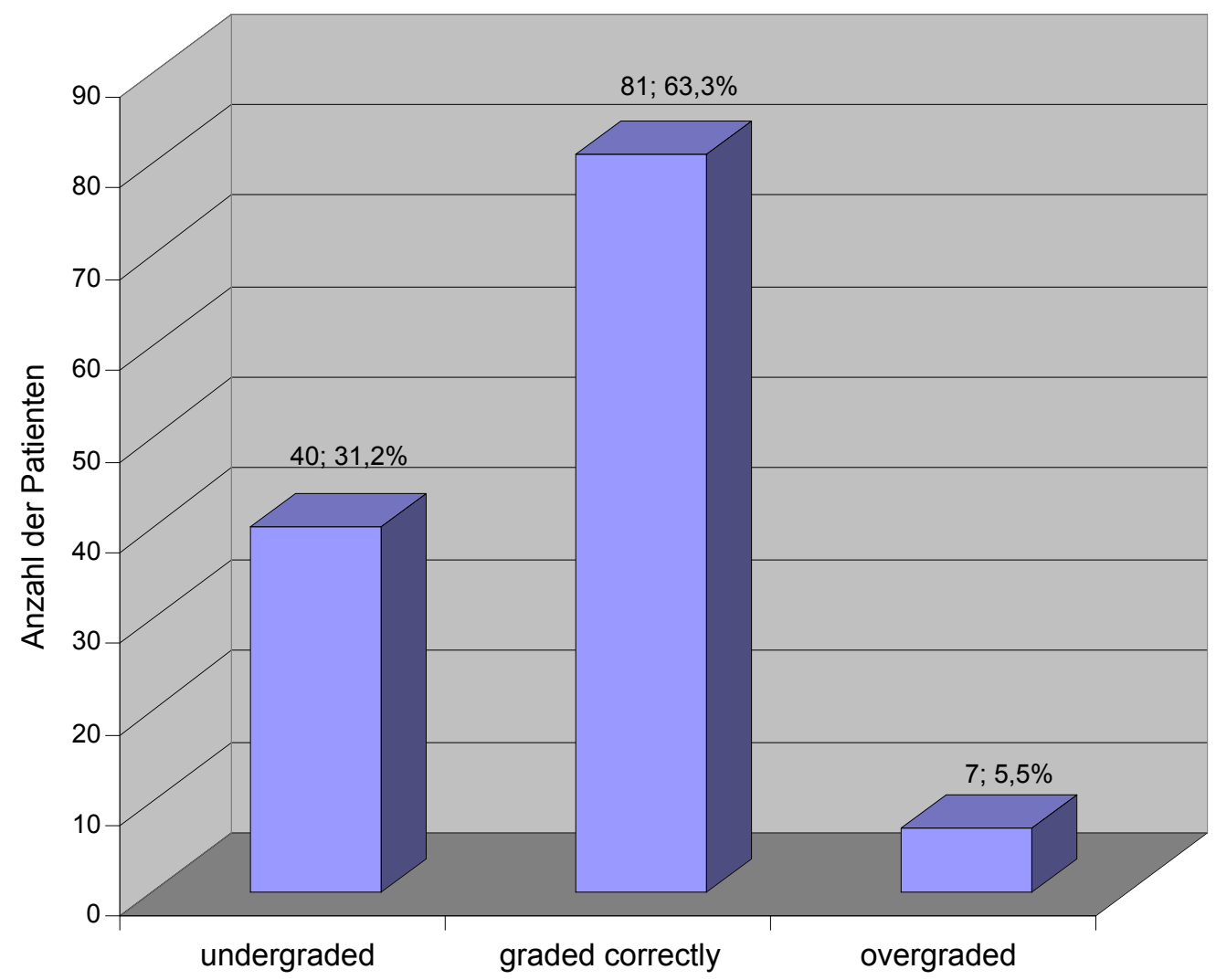

Abb.19: Vergleich des präoperativen Gradings mit dem postoperativen Ergebnis 
Betrachtet man das Grading in Abhängigkeit vom Alter so zeigt sich kein statistisch signifikanter Unterschied zwischen den Altersgruppen (Kruskal-Walis-Test mit $p=0,19)$.

\subsection{1 pT-Stadium in Abhängigkeit vom präoperativen Grading}

Bei dem Vergleich der Fälle mit organüberschreitendem Karzinom mit denen, die auf die Prostata begrenzt waren hinsichtlich des präoperativen Gradings, zeigt sich folgendes.

Das Grading der organbegrenzten Tumore unterscheidet sich signifikant von dem der organüberschreitenden Karzinome (Mann-Whitney U Test mit p=0,019). Die höheren Tumorstadien weisen im Median ein höheres Grading auf.

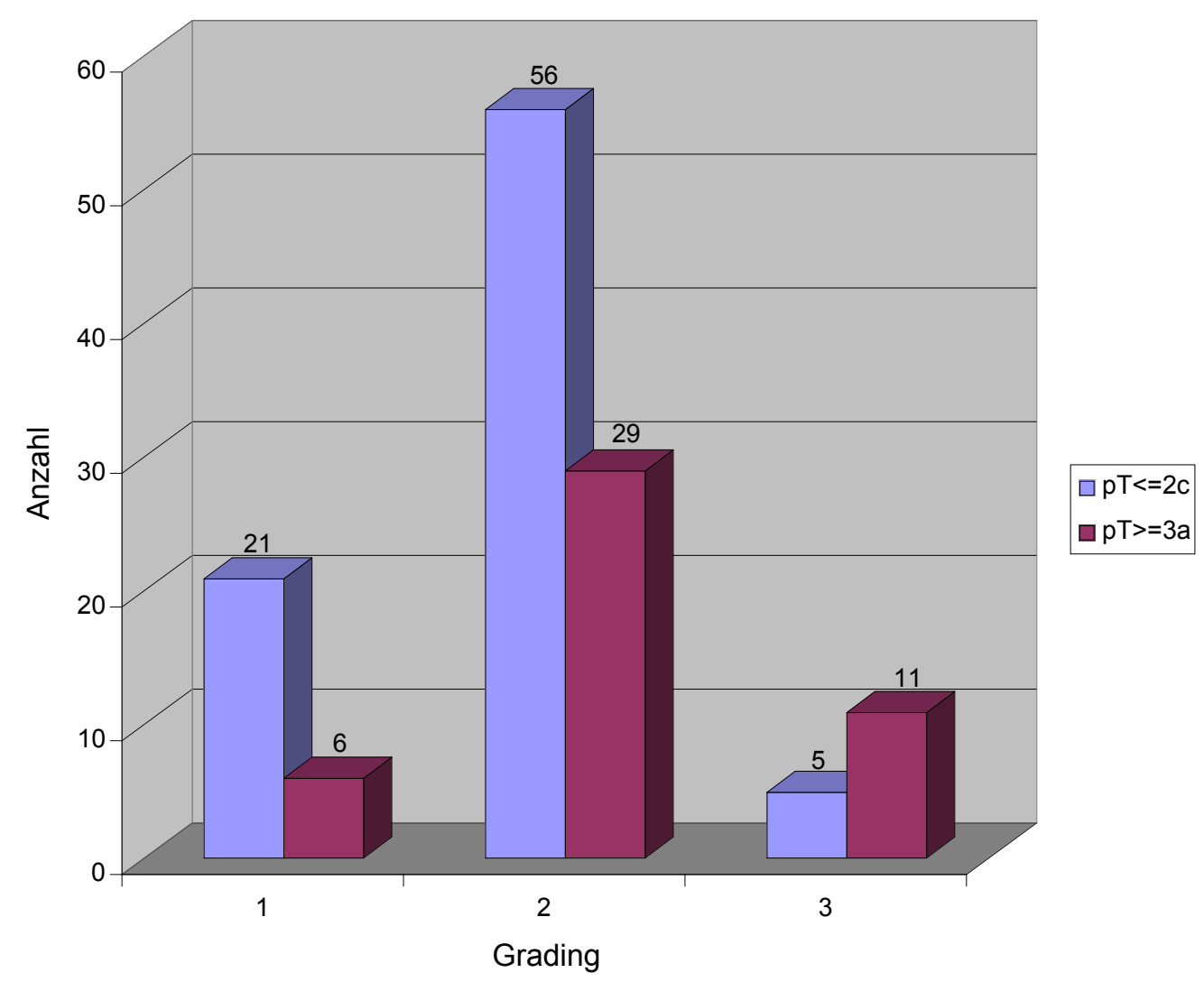

Abb.20: Vergleich der organbegrenzten und nicht organbegrenzten Tumore hinsichtlich des präoperativen Gradings

Ist bei Grad 1 nur ein Anteil von 22,3\% (6 von 27 Fällen) organüberschreitend, so steigt die Anzahl über Grad 2 mit 34,2\% (29 von 85 Fällen) auf 69\% (11 von 16 Fällen) bei Grad 3 (Abb.20). 


\subsubsection{Absetzungsrand in Abhängigkeit vom präoperativen Grading}

Betrachtet man das präoperative Grading bei positivem gegenüber negativem $\mathrm{Ab}$ setzungsrand, zeigt sich, dass der Unterschied des Gradings der R0-resezierten Patienten zu den R1-resezierten Patienten nicht signifikant ist (Mann-Whitney U Test mit $\mathrm{p}=0,099)$.

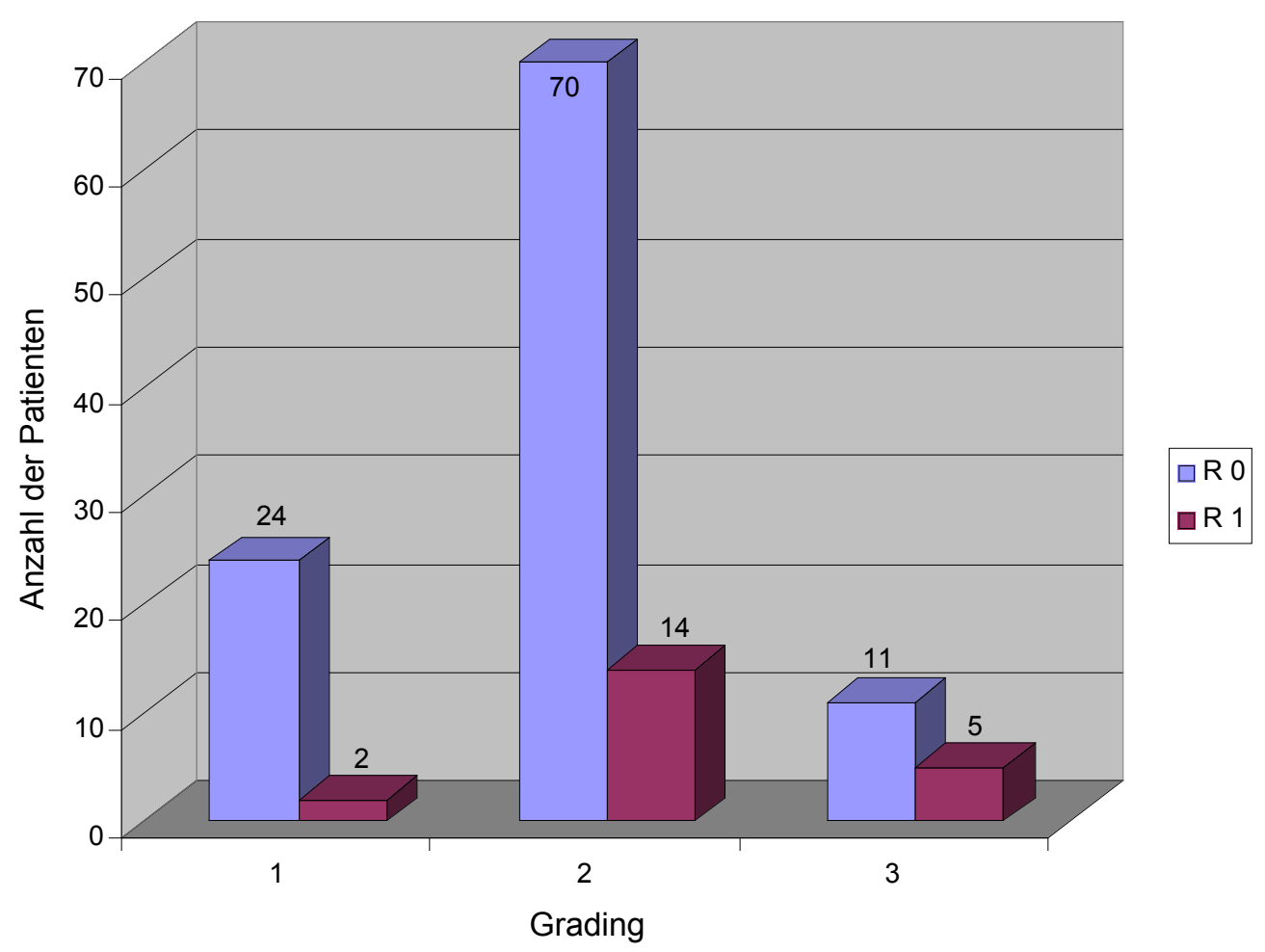

Abb.21: Vergleich von positivem und negativem Absetzungsrand hinsichtlich des präoperativen Gradings

Bei Grad 1 findet sich ein Anteil von 7,7\% (2 von 26 Fällen) mit positivem Absetzungsrand, bei Grad 2 sind es 16,7\% (14 von 84 Fällen) und bei Grad 3 ist der Anteil der R1-resezierten 31,3\% (5 von 16 Fällen) (Abb.21).

\subsubsection{Lymphknotenstatus in Abhängigkeit vom Grading}

Betrachtet man das Verhältnis der lymphknotenpositiven zu den lymphknotennegativen Fällen hinsichtlich des präoperativen Gradings, so zeigt sich folgendes.

Das Grading der Lymphknotennegativen unterscheidet sich signifikant von dem der Patienten mit Lymphknotenmetastasen (Mann-Whitney U Test mit $p=0,017$ ). 
Ein positiver Lymphknotenstatus geht im Median mit einem höheren Grading einher, als ein negativer Lymphknotenstatus.

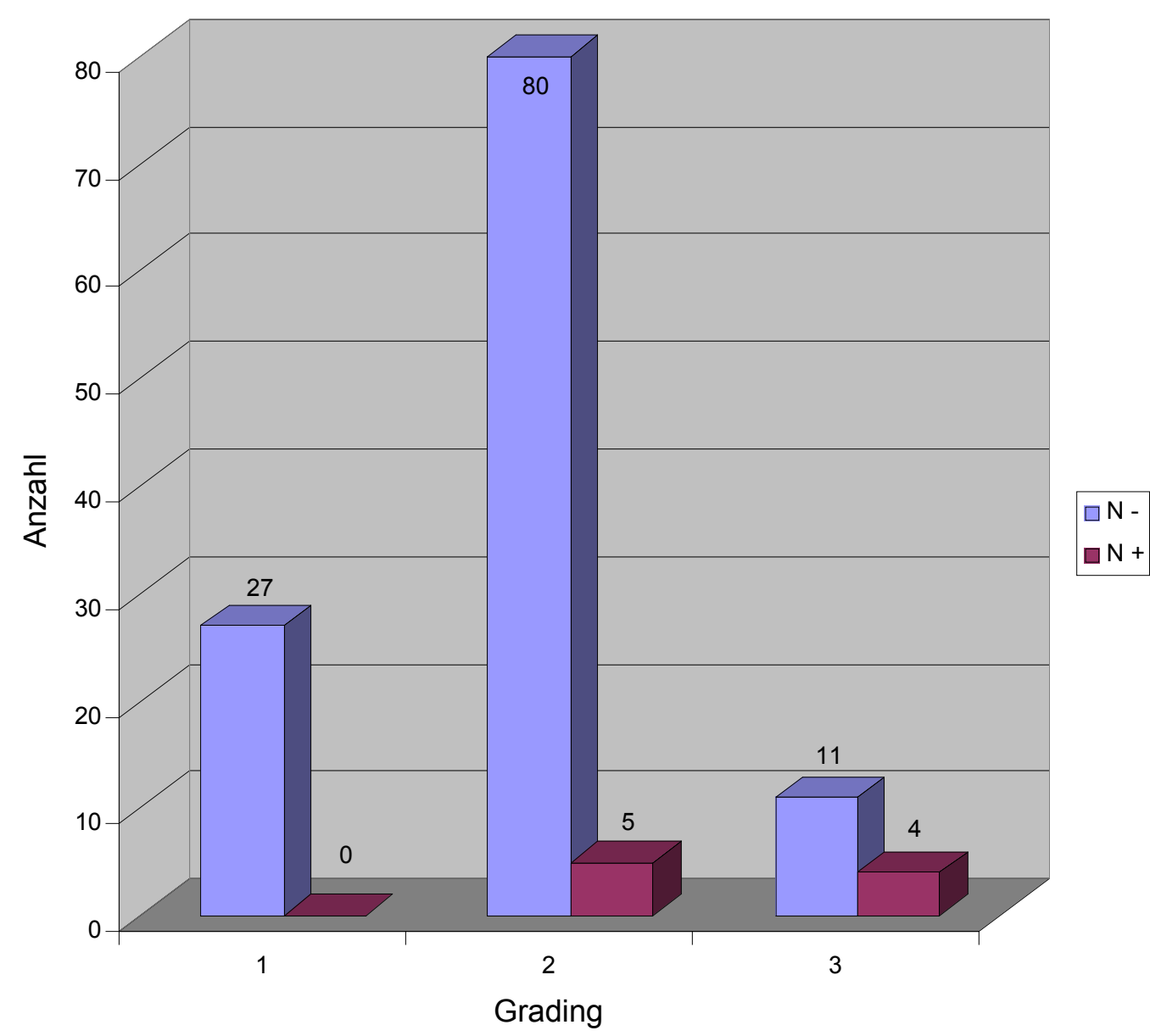

Abb.22: Vergleich von positivem und negativem Lymphknotenstatus hinsichtlich des präoperativen Gradings

Bei allen 27 Fällen, bei denen ein Grad 1 Tumor präoperativ diagnostiziert wurde, hat man keinen positiven Lymphknoten gefunden. Bei den Grad-2-Tumoren findet man bei einem Anteil von 5,9\% (5 von 85 Fällen) positive Lymphknoten und bei Grad 3 steigt der Anteil auf 26,7\% (4 von 15 Fällen) (Abb.22).

\subsection{Analyse der Daten hinsichtlich des Gleason-Scores}

Die histologische Einstufung nach Gleason lag präoperativ in 115 Fällen (82,2\%) vor. Einen Score von 5-6 erhielten die meisten Gewebeproben $(n=64 ; 55,7 \%)$. In 23 Fällen (20\%) war histologische Differenzierung des Prostatagewebes mit 
Gleason 2-4 noch nicht so stark reduziert. Bei 9 Beurteilungen (7,8\%) wurden ein Gleason- Score von $7(3+4)$, bei $7(6,1 \%)$ einer von $7(4+3)$ und in 12 Fällen $(10,4 \%)$ ein Score von 8-10 festgestellt (Tab.12).

\begin{tabular}{|c|c|c|}
\hline Gleason- Score & $\begin{array}{c}\text { Präoperativ } \\
\text { Anzahl Patienten }(\%)\end{array}$ & $\begin{array}{c}\text { Postoperativ } \\
\text { Anzahl Patienten (\%) }\end{array}$ \\
\hline $\mathbf{2}-\mathbf{4}$ & $23(20)$ & $9(7,9)$ \\
\hline $\mathbf{5 - 6}$ & $64(55,7)$ & $52(45,6)$ \\
\hline $\mathbf{3 + 4 = 7}$ & $9(7,8)$ & $8(15,8)$ \\
\hline $\mathbf{4 + 3}=\mathbf{7}$ & $7(6,1)$ & $27(23,7)$ \\
\hline $\mathbf{8}-\mathbf{1 0}$ & $12(10,4)$ & $114(100)$ \\
\hline Gesamt & $115(100)$ & $8(7)$ \\
\hline
\end{tabular}

Tab.12: Aufteilung der Fälle nach der histologischen Beurteilung nach Gleason prä- und postoperativ

Postoperativ wurden 114 Prostatapräparate $(81,4 \%)$ histologisch beurteilt. Noch relativ differenziertes Gewebe fand sich bei 9 Prostatapräparaten $(7,9 \%)$ mit einem Score von 2-4. Weniger differenziert war das Prostatagewebe von 52 Patienten $(45,6 \%)$ bei Gleason 5-6, von 18 Patienten $(15,8 \%)$ bei Gleason $7(3+4)$ und von 8 Patienten (7\%) bei Gleason 7(4+3). Mäßig bis schlecht differenziert (Gleason 8-10) fand der Pathologe die Prostatapräparate in 27 Fällen (23,7\%) vor (Tab.12).

Vergleicht man die präoperativ ermittelten Gleason-Scores mit der definitiven postoperativen Klassifikation nach Gleason, so stimmte der aus den Stanzzylindern bestimmte Gleason-Score in 34 Fällen (34,7\%) mit dem postoperativen Ergebnis überein. 51 Tumore (52\%) wurden präoperativ zu niedrig und 13 Tumore (13,3\%) wurden zu hoch klassifiziert (Abb.23). 


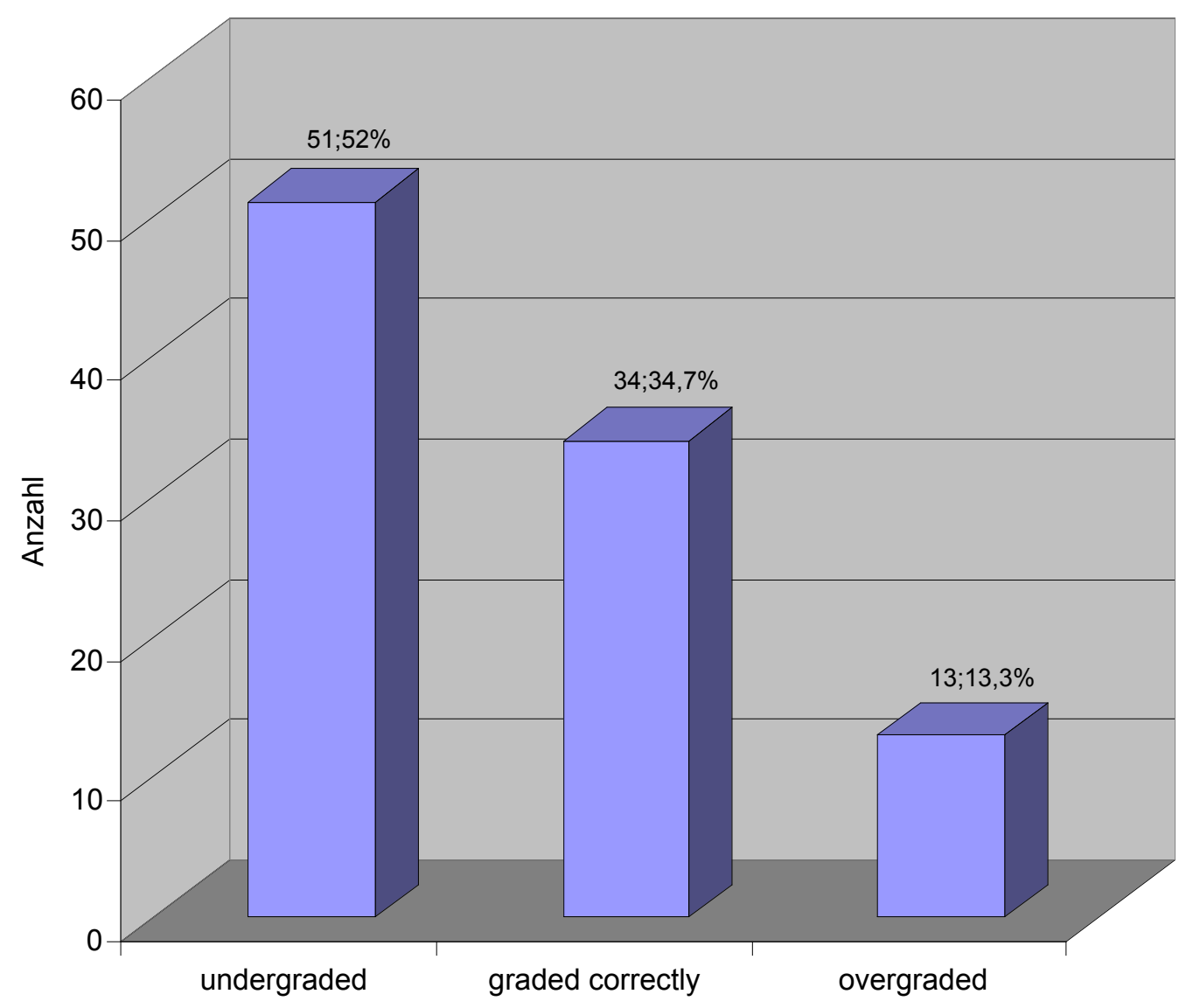

Abb.23: Vergleich des präoperativen Gleason-Scores mit der postoperativen Klassifikation nach Gleason

Im Folgenden wurden die Ergebnisse zum Gleason-Score noch einmal tabellarisch verdeutlicht. Hervorgehoben sind die prä- und postoperativ übereinstimmenden Fälle (34,7\%), sowie die Übereinstimmung des Gleason-Scores +/-1 Grad $(69,4 \%)$ (Tab.:13). 


\begin{tabular}{|c|c|c|c|c|c|c|c|c|c|c|}
\hline \multirow{2}{*}{$\begin{array}{l}\text { Gleason- } \\
\text { Score } \\
\text { präoperativ }\end{array}$} & \multicolumn{9}{|c|}{ Gleason-Score postoperativ, $\mathrm{n}$} & \multirow[b]{2}{*}{$\begin{array}{l}\text { Total, } \mathrm{n} \\
(\%)\end{array}$} \\
\hline & 2 & 3 & 4 & 5 & 6 & 7 & 8 & 9 & 10 & \\
\hline 2 & - & - & - & 2 & 1 & - & - & - & - & $3(3)$ \\
\hline 3 & - & $1 *$ & - & 2 & 1 & 1 & - & - & - & $5(5)$ \\
\hline 4 & - & $1 * *$ & $2^{*}$ & - & 4 & - & 2 & - & - & $9(9)$ \\
\hline 5 & - & 1 & - & $3 *$ & $10 * *$ & - & - & 1 & - & $15(15,5)$ \\
\hline 6 & - & - & 2 & $1 * *$ & $19 *$ & $13 * *$ & 1 & 4 & - & $40(41)$ \\
\hline 7 & - & - & - & 2 & $1 * *$ & $7 *$ & $4 * *$ & 2 & - & $16(16,5)$ \\
\hline 8 & - & - & - & - & - & $2 * *$ & $1 *$ & - & - & $3(3)$ \\
\hline 9 & - & - & - & - & - & - & $2 * *$ & $4^{*}$ & - & $6(6)$ \\
\hline 10 & - & - & - & - & - & - & - & - & $1^{*}$ & $1(1)$ \\
\hline $\begin{array}{ll}\text { Total, } & \mathrm{n} \\
(\%) & \end{array}$ & - & $\begin{array}{c}3 \\
(3)\end{array}$ & $\begin{array}{l}4 \\
(4)\end{array}$ & $\begin{array}{c}10 \\
(10,3)\end{array}$ & $\begin{array}{c}36 \\
(36,6)\end{array}$ & $\begin{array}{c}23 \\
(23,4)\end{array}$ & $\begin{array}{c}10 \\
(10,3)\end{array}$ & $\begin{array}{c}11 \\
(11,4)\end{array}$ & $\begin{array}{c}1 \\
(1)\end{array}$ & $\begin{array}{rr}98 & \\
& (100)\end{array}$ \\
\hline
\end{tabular}

$*=$ graded correctly; $n=34(34,7 \%), \quad * *=$ graded correctly $+/-1 ; n=68(69,4 \%)$

Tab. 13: Gesamtkorrelation des prä- zum postoperativen Gleason-Score 


\subsubsection{Der präoperative Gleason-Score in Abhängigkeit vom Alter}

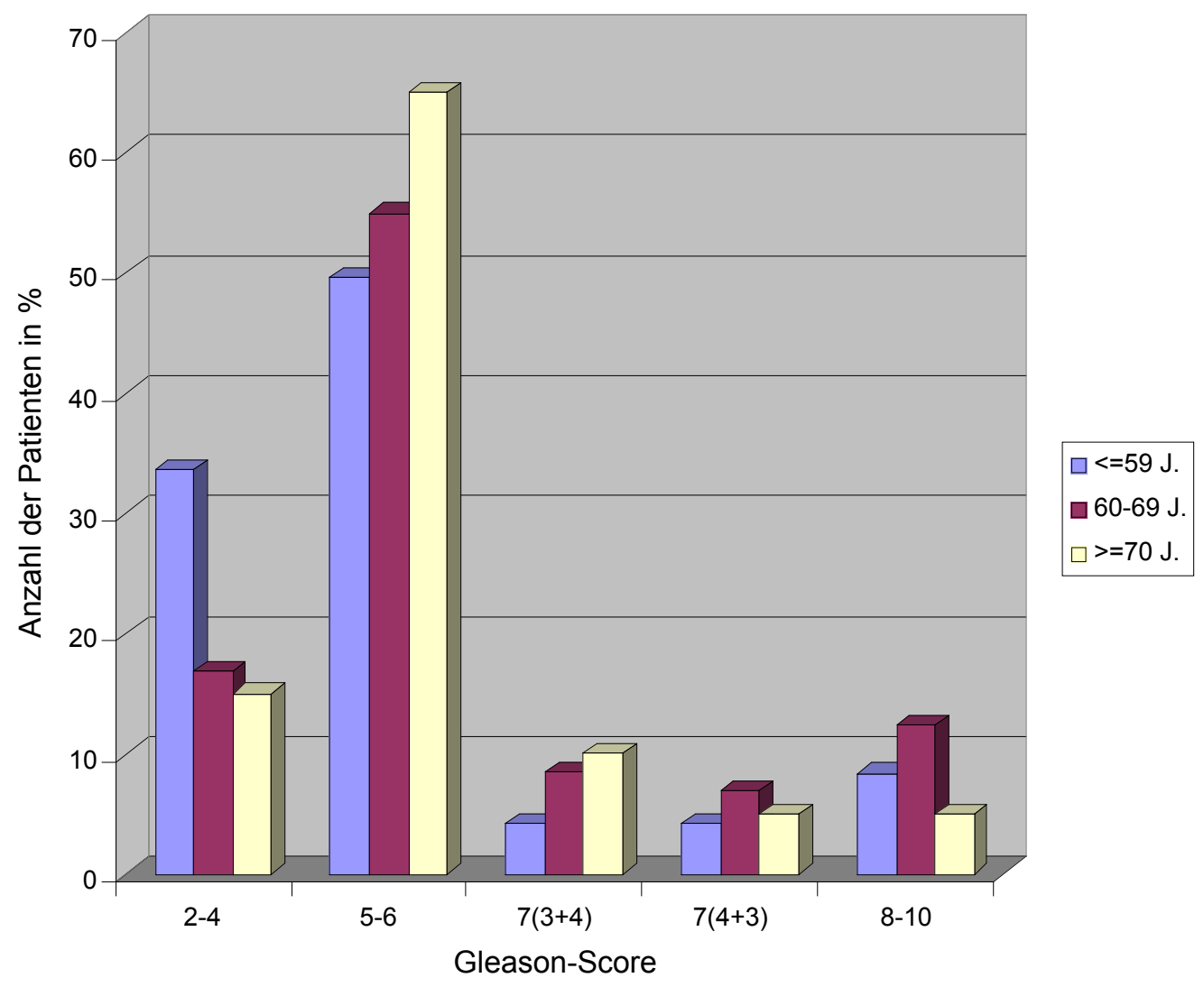

Abb.24: Gleason-Score in Abhängigkeit vom Lebensalter

Betrachtet man den Gleason-Score in Abhängigkeit vom Alter der Patienten, so muss festgestellt, dass das Alter keinen Einfluss auf die Höhe des Gradings nach Gleason hat. Der Unterschied ist statistisch nicht signifikant (Kruskal-Wallis-Test mit $\mathrm{p}=0,194)$.

\subsection{2 pT-Stadium in Abhängigkeit vom Gleason-Score}

Im Folgenden wurden die Fälle mit organüberschreitendem Karzinom mit denen, die auf die Prostata begrenzt waren, hinsichtlich des präoperativen GleasonScores verglichen. Der Gleason-Score der organbegrenzten Tumore unterscheidet sich hochsignifikant von dem der organüberschreitenden Karzinome (MannWhitney U Test mit $\mathrm{p}=0,006$ ). Letztere zeigten im Median einen wesentlich höheren Gleason-Score. 


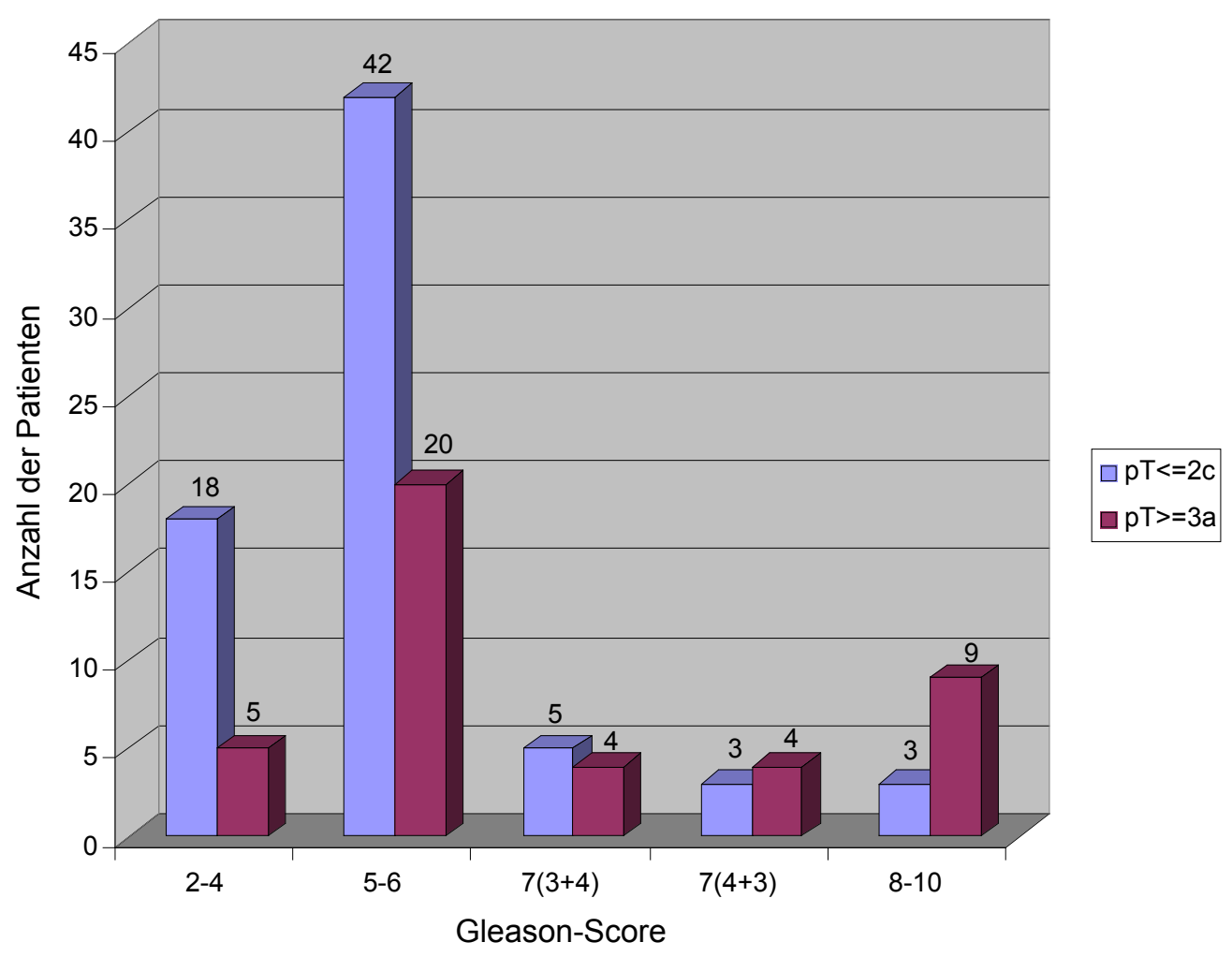

Abb.25: Vergleich der organbegrenzten und nicht organbegrenzten Tumore hinsichtlich des präoperativen Gleason-Scores

Ist bei einem Gleason-Score von 2-4 nur ein Anteil von 21,7\% (5 von 18 Fällen) organüberschreitend, so steigt die Anzahl über Gleason 5-6 mit 22,3\% (20 von 42 Fällen), über Gleason 7(3+4) mit 44,4\% (4 von 5 Fällen) und über Gleason 7(4+3) mit 57,1\% (4 von 7 Fällen) auf 75\% (9 von 12 Fällen) bei einem Gleason-Score von 8-10 (Abb.25).

\subsubsection{Absetzungsrand in Abhängigkeit vom Gleason-Score}

Betrachtet man den präoperativen Gleason-Score bei positivem gegenüber negativem Absetzungsrand, zeigt es sich, dass der Gleason-Score der R0-resezierten Tumore sich signifikant von dem der R1-resezierten Karzinome unterscheidet (Mann-Whitney U Test mit p=0,017). Ein positiver Absetzungsrand geht im Median mit einem höheren Gleason-Score einher. 


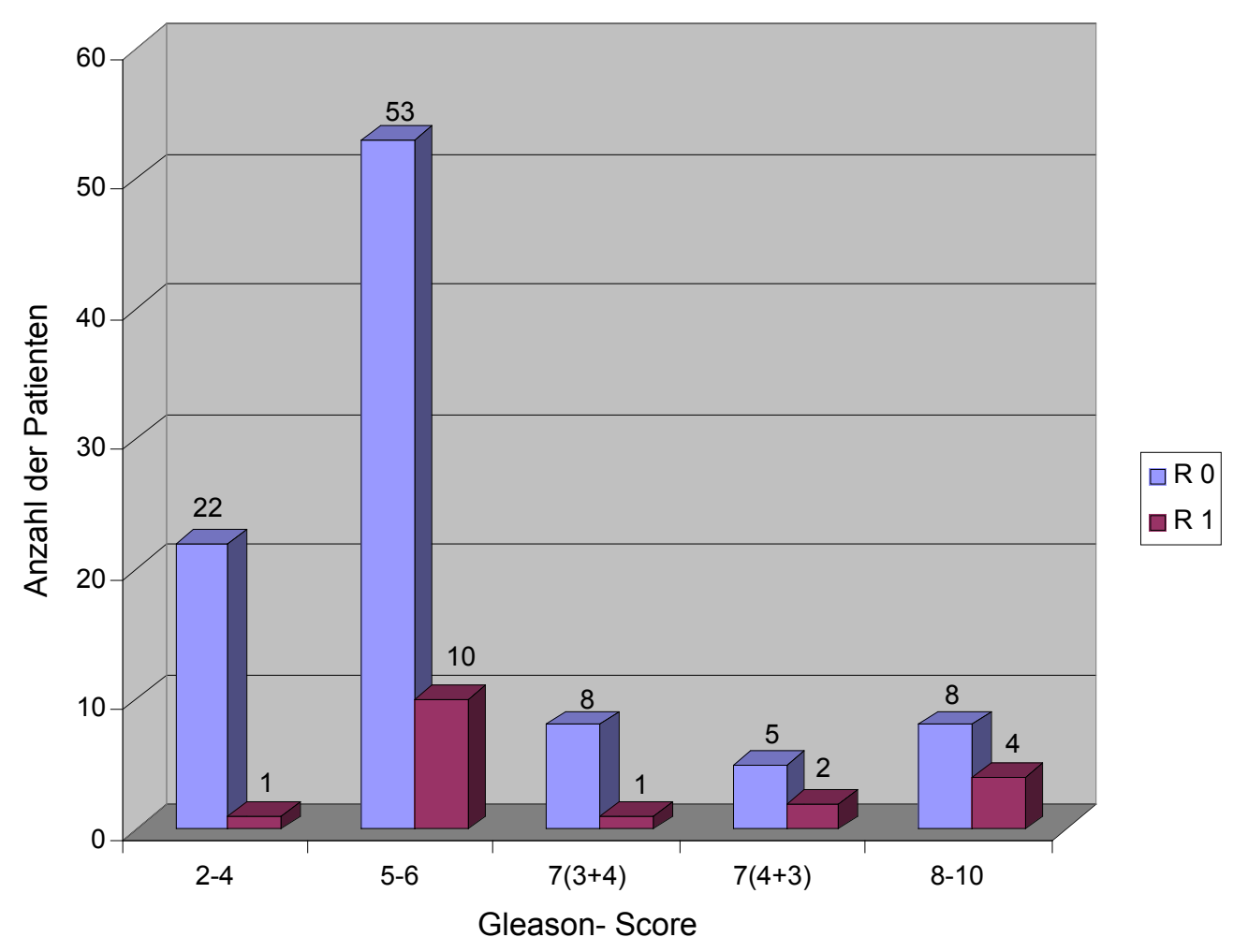

Abb.26: Vergleich von positivem und negativem Absetzungsrand hinsichtlich des präoperativen Gleason-Scores

Bei den Gruppen im Einzelnen findet sich bei Gleason 2-4 ein Anteil von 4,3\% (1 von 22 Fällen) mit positivem Absetzungsrand, bei Gleason 5-6 sind es schon 15,9\% (10 von 63 Fällen), bei Gleason 7(3+4) sinkt der Anteil auf 11,1\% (1 von 9 Fällen), woraufhin bei Gleason 7(4+3) der Anteil wieder auf 28,6\% (2 von 7 Fällen) steigt. Schließlich ist bei der Gleason-Gruppe 8-10 der höchste Anteil mit 33,3\% (4 von 12 Fällen) zu verzeichnen (Abb.26).

\subsubsection{Lymphknotenstatus in Abhängigkeit vom Gleason-Score}

Der Gleason-Score der Patienten mit negativem Lymphknotenstatus unterscheidet sich hochsignifikant von dem der Patienten mit positivem Lymphknotenstatus (Mann-Whitney U Test mit $\mathrm{p}=0$,004). Letztere Gruppe weist im Median einen wesentlich höheren Gleason-Score auf. 


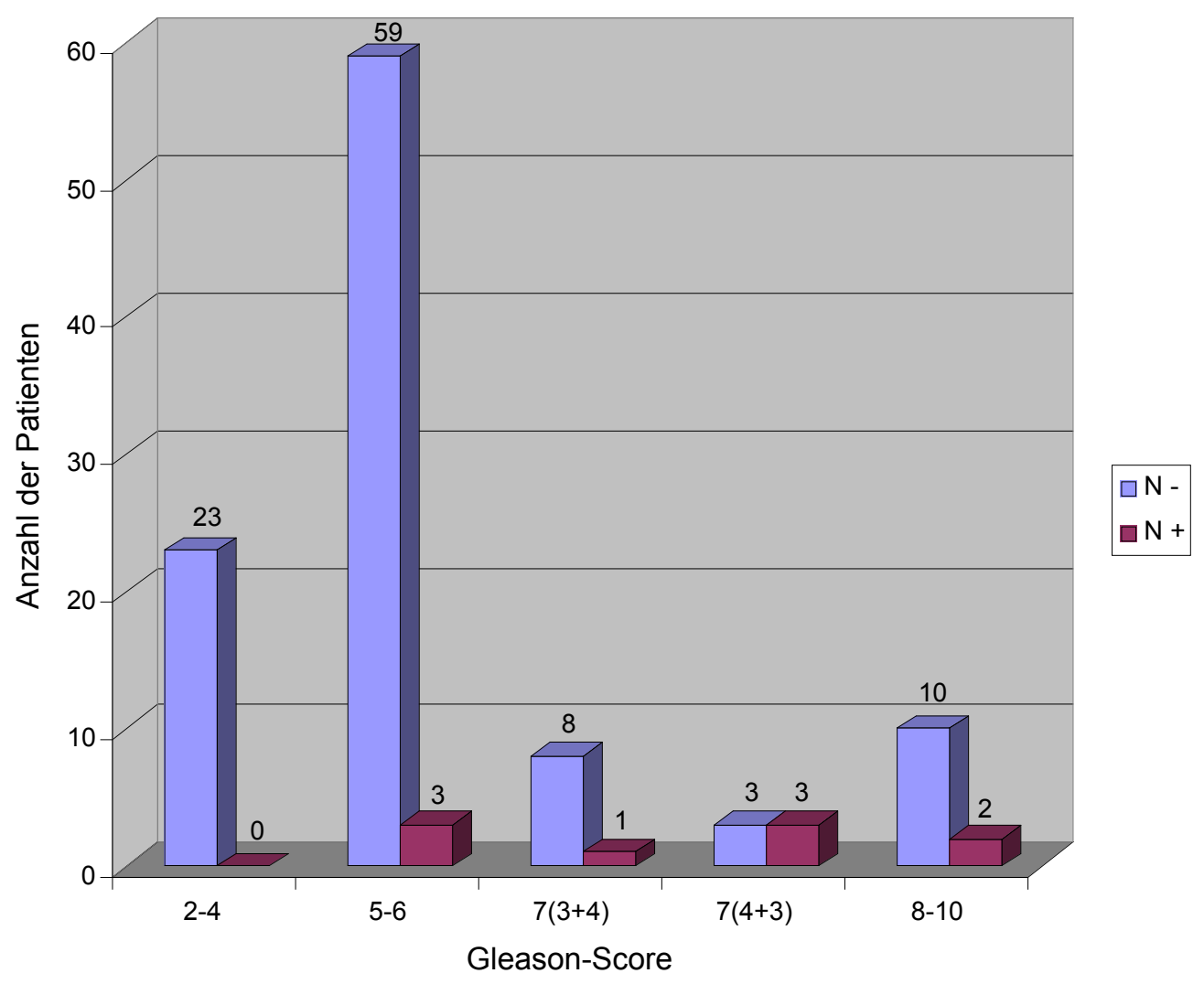

Abb.27: Vergleich von positivem und negativem Lymphknotenstatus hinsichtlich des präoperativen Gleason-Scores

Bei allen 23 Fällen der Gleason-Gruppe Grad 2-4 hat man keinen positiven Lymphknoten gefunden. Bei Gleason 5-6 findet man einen Anteil von 4,8\% (3 von 62 Fällen), bei Gleason 7(3+4) einen Anteil von 11,1\% (1 von 9 Fällen) und bei Gleason 7(4+3) steigt der Anteil auf 50\% (3 von 6 Fällen) positive Lymphknoten. In der Gruppe mit Gleason-Score 8-10 nimmt der Anteil der positiven Lymphknoten mit 16,7\% (2 von 12 Fällen) wieder etwas ab (Abb.27).

\subsection{Analyse der Daten hinsichtlich des präoperativen PSA- Wertes}

\subsubsection{Einteilung der Patienten nach PSA-Wert}

In allen 140 Fällen (100 \%) konnte der präoperative PSA-Wert ermittelt werden. 
Die Gruppen wurden wie folgt verteilt (Abb.28): PSA $<3,9 \mathrm{ng} / \mathrm{ml} \quad(\mathrm{n}=10)$

PSA 4 bis $9,9 \mathrm{ng} / \mathrm{ml} \quad(\mathrm{n}=70)$

PSA 10 bis $19,9 \mathrm{ng} / \mathrm{ml}(\mathrm{n}=45)$

PSA $>=20 \mathrm{ng} / \mathrm{ml} \quad(\mathrm{n}=15)$

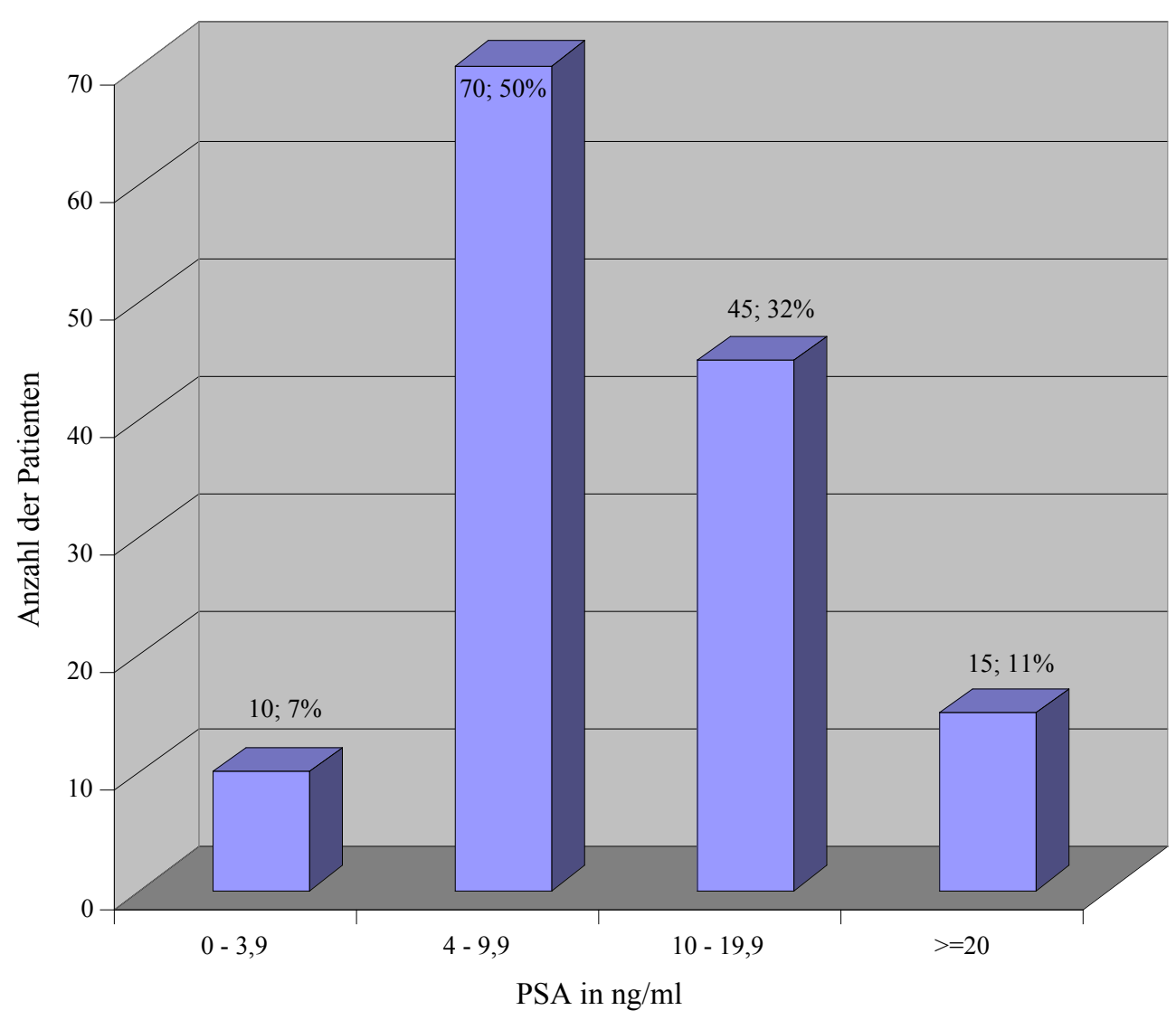

Abb.28: Verteilung der Patienten in die PSA-Gruppen

Betrachtet man den PSA-Wert in Abhängigkeit vom Alter, so lässt sich kein signifikanter Unterschied feststellen (Kruskal-Walis-Test mit $\mathrm{p}=0,197$ ). 


\subsection{2 pT-Stadium in Abhängigkeit vom PSA-Wert}

Im Folgenden wurden die Fälle mit organüberschreitendem Karzinom mit denen, die auf die Prostata begrenzt waren, hinsichtlich des präoperativen PSA-Wertes verglichen.

Der PSA-Wert der organbegrenzten Tumore unterscheidet sich hochsignifikant von dem der organüberschreitenden Karzinome (Mann-Whitney-U-Test mit $\mathrm{p}=0,009)$. Bei den höheren Tumorstadien wurde im Median auch ein höherer PSA-Wert gemessen.

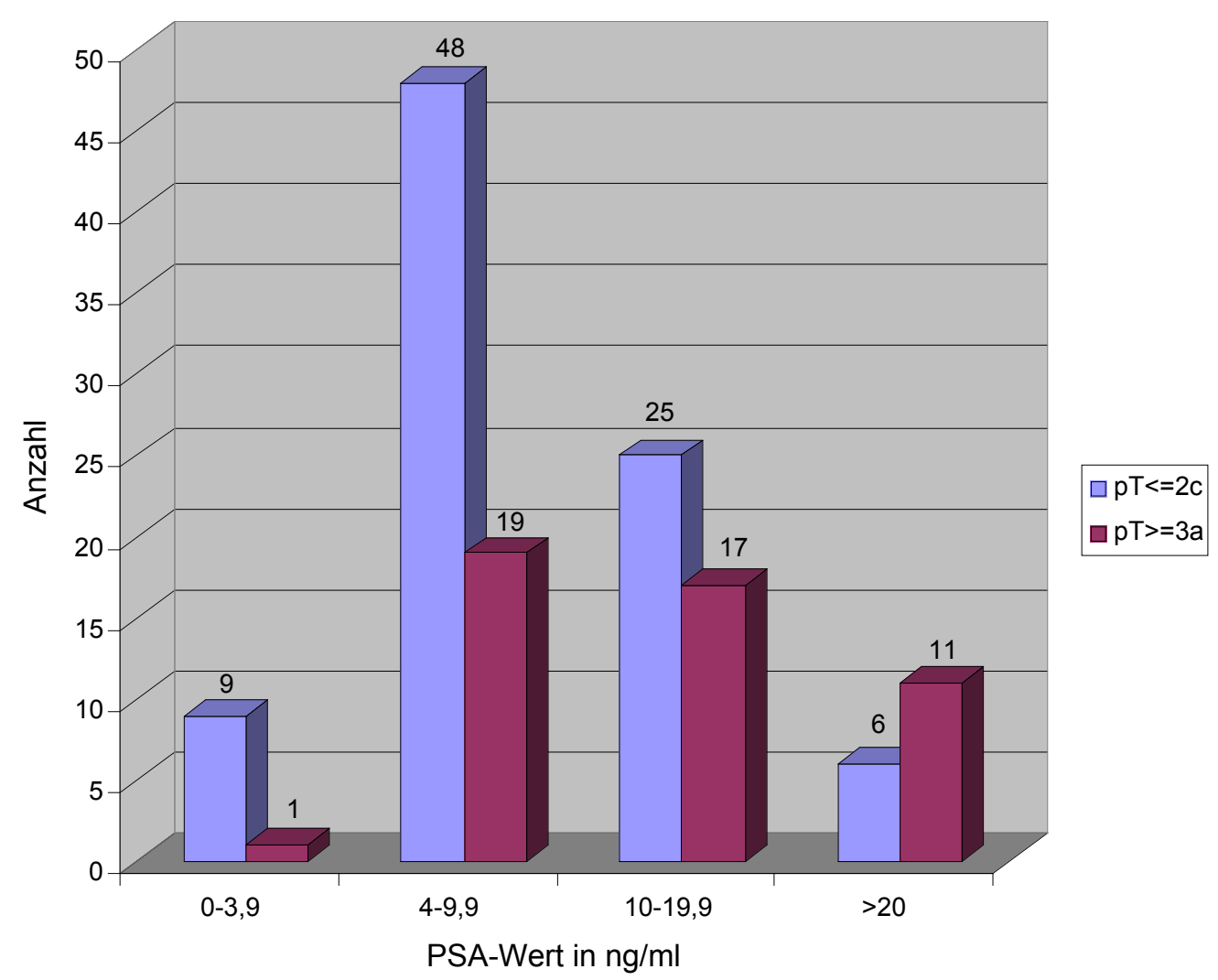

Abb.29: Vergleich der organbegrenzten und nicht organbegrenzten Tumore hinsichtlich des präoperativen PSA-Wertes

Ist bei einem PSA von 0-3,9 ng/ml nur ein Anteil von 10\% (1 von 10 Fällen) organüberschreitend, so steigt die Anzahl über die PSA-Gruppe 4-9,9 ng/ml mit 28,4\% (19 von 67 Fällen) und über PSA 10-19,9 ng/ml mit 40,5\% (17 von 42 Fällen) auf 64,7\% (11 von 17 Fällen) bei der PSA-Gruppe $>=20$ ng/ml (Abb.29). 


\subsubsection{Absetzungsrand in Abhängigkeit vom PSA-Wert}

Das PSA-Spiegel der R0-resezierten Patienten unterscheidet sich nicht signifikant von dem der R1-resezierten Patienten (Mann-Whitney-U-Test mit p=0,475).

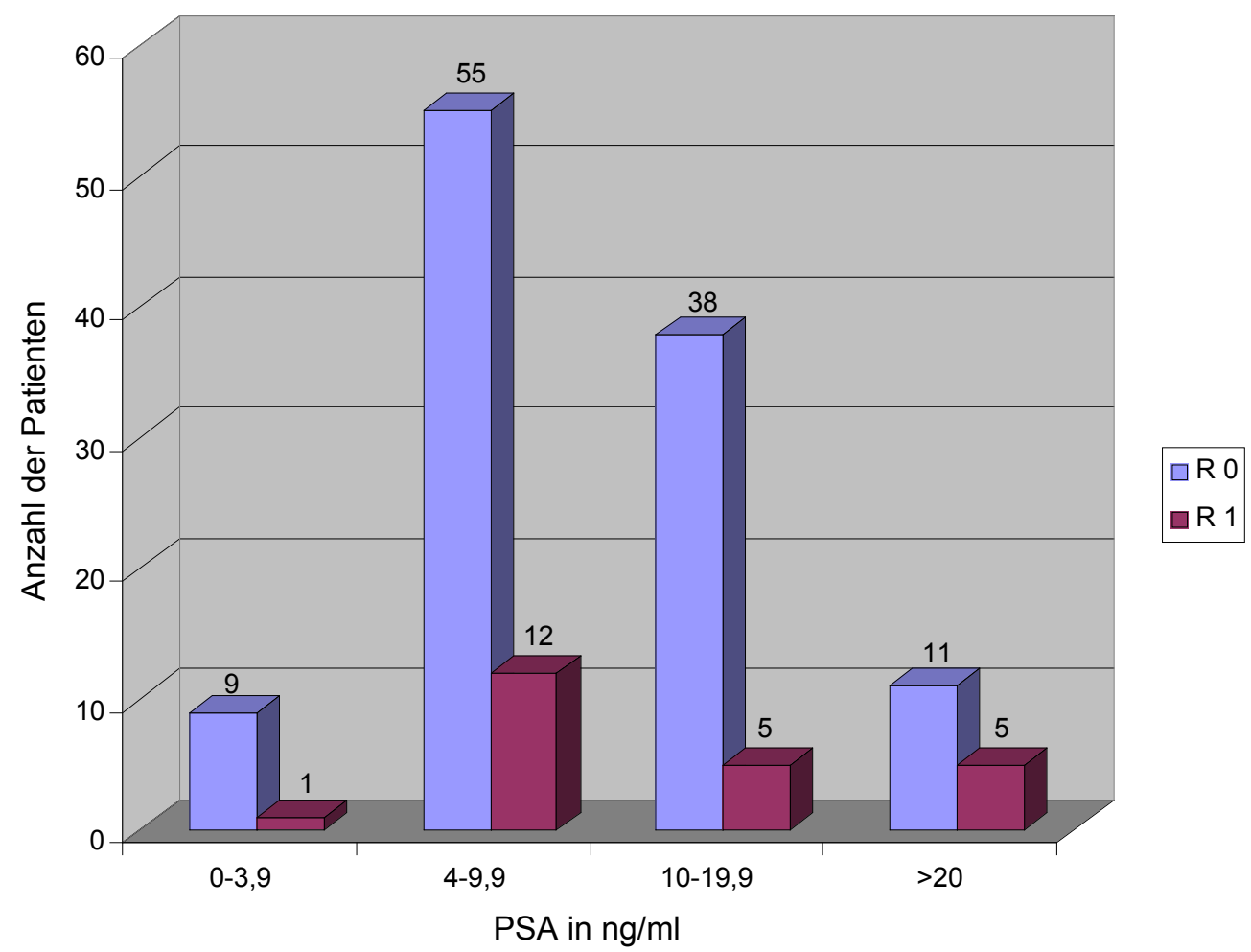

Abb.30: Vergleich von positivem und negativem Absetzungsrand hinsichtlich des präoperativen PSA-Wertes

Bei den Gruppen im Einzelnen findet sich bei PSA 0-3,9 ng/ml ein Anteil von 10\% (1 von 10 Fällen) mit positivem Absetzungsrand, bei der PSA-Gruppe 4-9,9 ng/ml sind es 18\% (12 von 67 Fällen), bei PSA 10-19,9 ng/ml sinkt der Anteil auf $11,6 \%$ ( 5 von 43 Fällen), woraufhin bei der PSA-Gruppe >=20ng/ml der Anteil wieder auf $31,3 \%$ (5 von 16 Fällen) steigt (Abb.30). 


\subsubsection{Lymphknotenbefall in Abhängigkeit vom PSA-Wert}

Der PSA-Wert der Patienten mit negativem Lymphknotenstatus unterscheidet sich signifikant von dem der Patienten mit positivem Lymphknotenstatus (MannWhitney-U-Test mit p=0,013). Die Häufigkeit des Lymphknotenbefalls nimmt mit steigendem PSA-Wert zu.

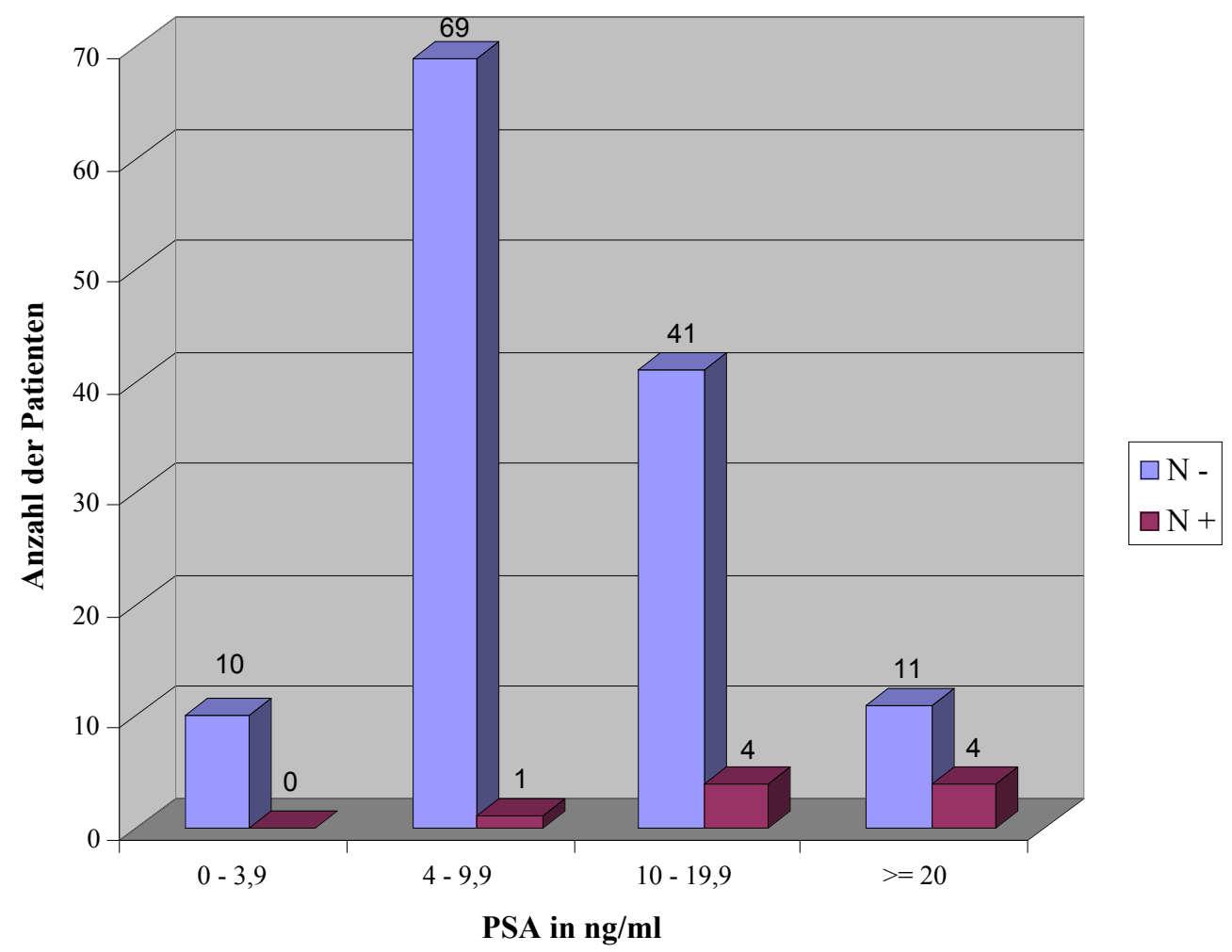

Abb.31: Lymphknotenstatus in Abhängigkeit vom PSA-Wert

Beim Patientenkollektiv mit präoperativen PSA-Werten zwischen 0 und 3,9 ng/ml ist kein Fall mit positivem Lymphknoten nachweisbar. Während in der Gruppe der Patienten mit einem PSA zwischen 4 und 9,9 ng/ml 1,4\% (1 von 70 Fällen) einen positiven Lymphknotenstatus aufweist, sind in dem folgenden Patientenkollektiv (PSA 10-19,9 ng/ml) bereits 8,9\% (4 von 45 Fällen) lymphkotenpositiv. Diese steigende Tendenz lässt sich durch das Patientenkollektiv mit einem PSA $>=20$ untermauern. Hier sind bereits bei $26,7 \%$ (4 von 15 Fällen) positive Lymphknoten zu finden (Abb.31). 


\subsection{Operationsdaten}

\subsubsection{Anzahl der Erythrozytenkonzentrate}

In 38 Fällen (26,8\%) wurden intraoperativ Erythrozytenkonzentrate (EKs) verabreicht. Die Anzahl reicht von einem Ek in 3 Fällen, über 2 EKs in 18 Fällen und drei EKs in 6 Fällen, zu vier oder mehr EKs in 10 Fällen (Abb.32).

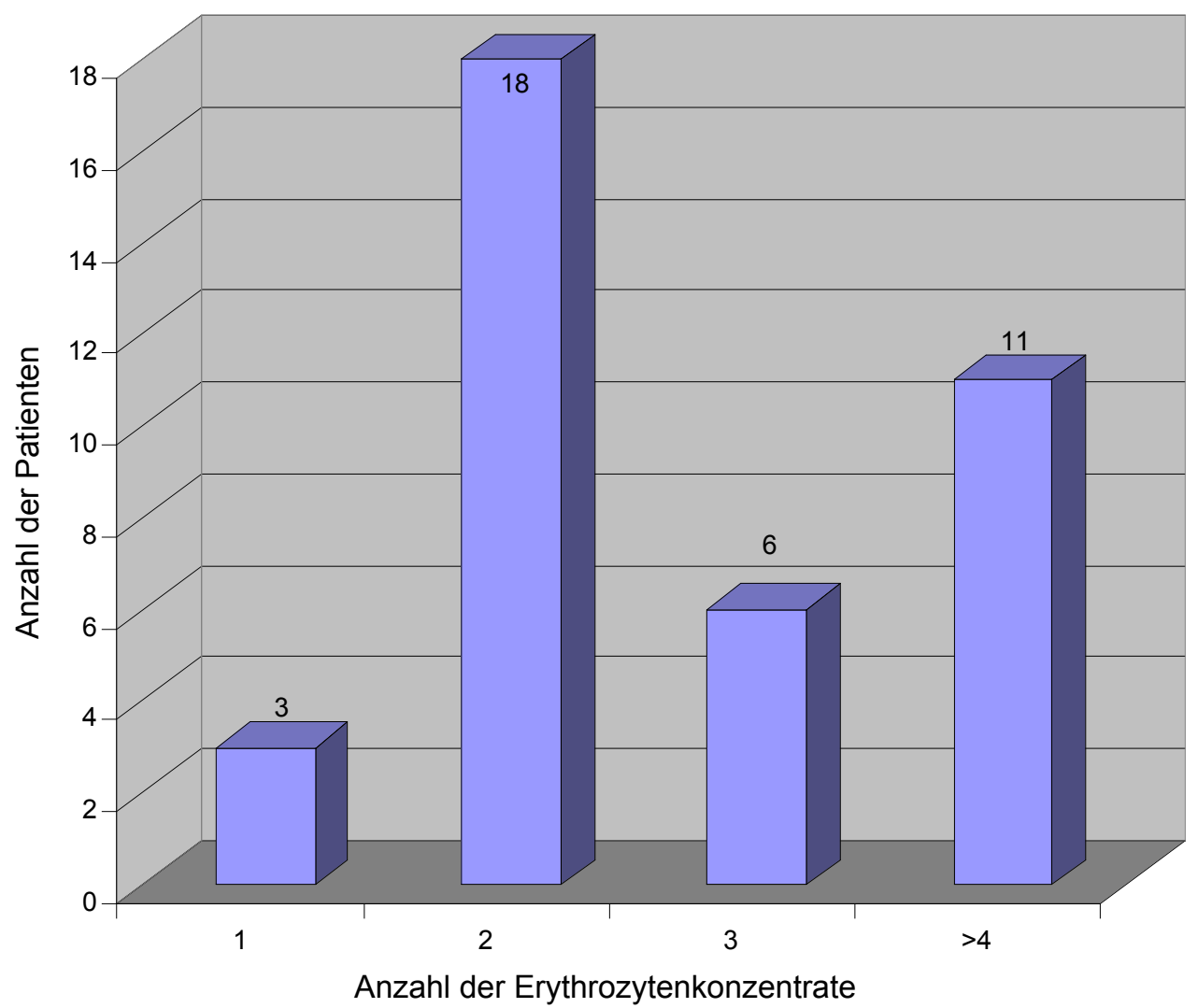

Abb.32: Übersicht über die Anzahl der intraoperativ verwendeten Erythrozytenkonzentrate

\subsubsection{Nervenschonende Operation}

Bei unserem Patientenkollektiv konnte in 70 Fällen (51\%) eine nervenschonende Prostatektomie durchgeführt werden. Das Ausmaß der Erkrankung bei den restlichen 49\% der Patienten genügte nicht den Kriterien, um das neurovaskuläre Bündel zu erhalten (Abb.33). 


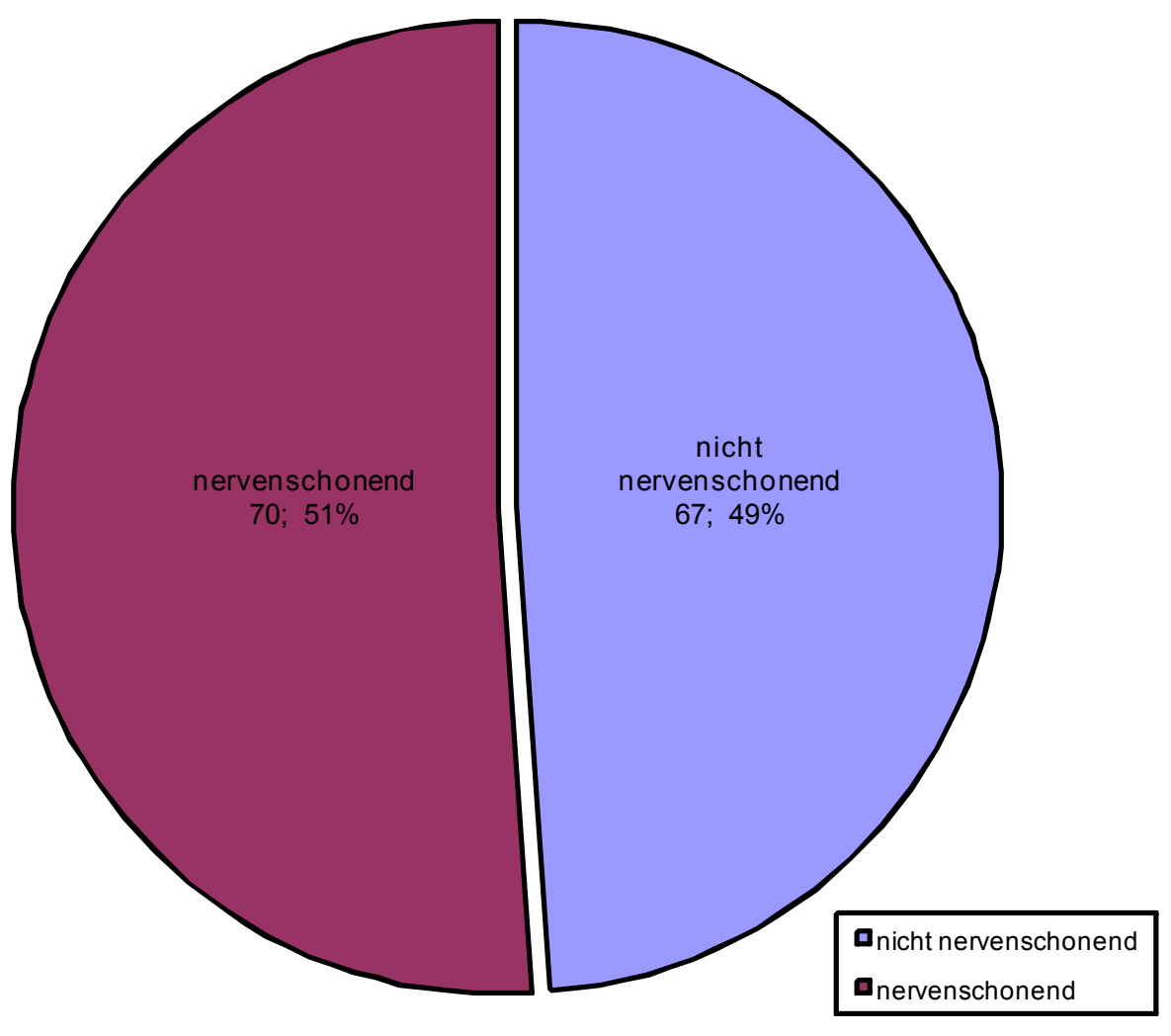

Abb.33: Anzahl der nervenschonend und nicht nervenschonend operierten Patienten

\subsection{Ergebnisse der Patientenbefragung}

Das Follow-up, also der Zeitraum nach der Prostatektomie bis zur Befragung im Rahmen der Studie ist bei den einzelnen Patienten unterschiedlich lang. Die kürzeste Beobachtungszeit beträgt 21 Monate, die längste 70 Monate. Das durchschnittliche Follow-up bei den 105 untersuchten Patienten beträgt 46,5 Monate. 


\subsubsection{Kontinenzstatus}

Durch den Fragebogen wurde der Kontinenzstatus der Patienten vor der Operation erhoben. Es zeigt sich, dass 100 Patienten (95\%) eine absolute Kontinenz (Grad 0) angeben.

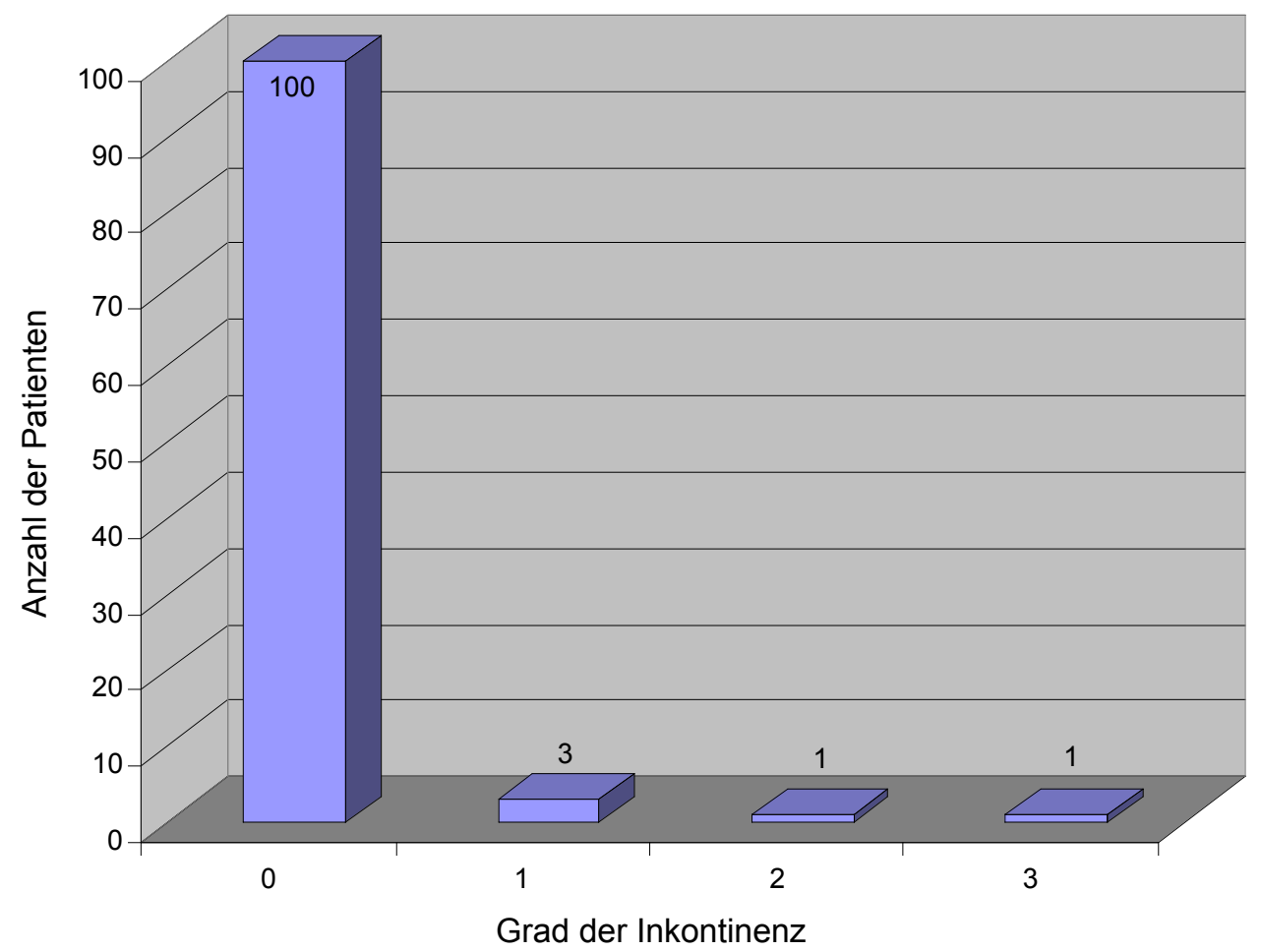

Abb.34: Kontinenzstatus der Patienten vor der Operation

Dieses Ergebnis deckt sich auch mit den Angaben der Patienten zu der Anzahl der Vorlagen, wo jeweils kein Gebrauch von Vorlagen angegeben wurde. Drei Patienten $(3 \%)$ gaben eine Inkontinenz Grad I, einer Grad II und ein weiterer eine Inkontinenz III an (Abb.34). 
Ebenfalls erfragt worden ist der Kontinenzstatus zum Zeitpunkt der Befragung, also nach der Operation.

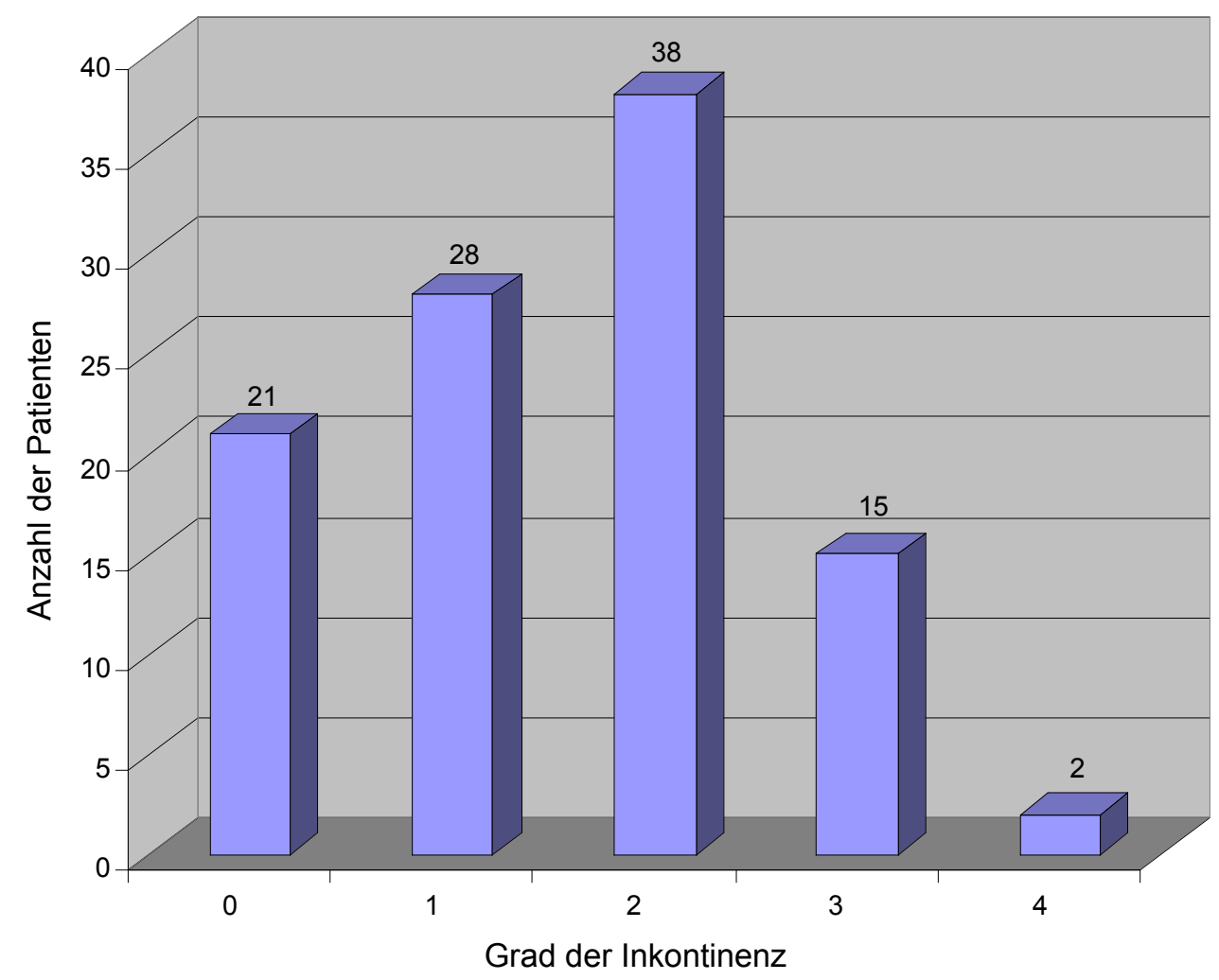

Abb.35: Kontinenzstatus der Patienten nach der Operation

Hier zeigt es sich, dass 21 Patienten (20\%) nach der Operation zum Zeitpunkt der Befragung absolut Kontinent ( Grad 0) sind. Bei der Inkontinenz Grad I steigt die Zahl auf 28 Operierte (27\%), Inkontinenz Grad II wird mit 38 (37\%) von den meisten Befragten angegeben, Grad III trifft auf 15 (15\%) Patienten zu, während 2 Patienten einen Inkontinenzgrad von IV angeben (Abb.35). 
Fragt man nach der Anzahl der Vorlagen, die der Patient innerhalb von 24 Stunden benutzt, zeigt sich folgende Aufteilung.

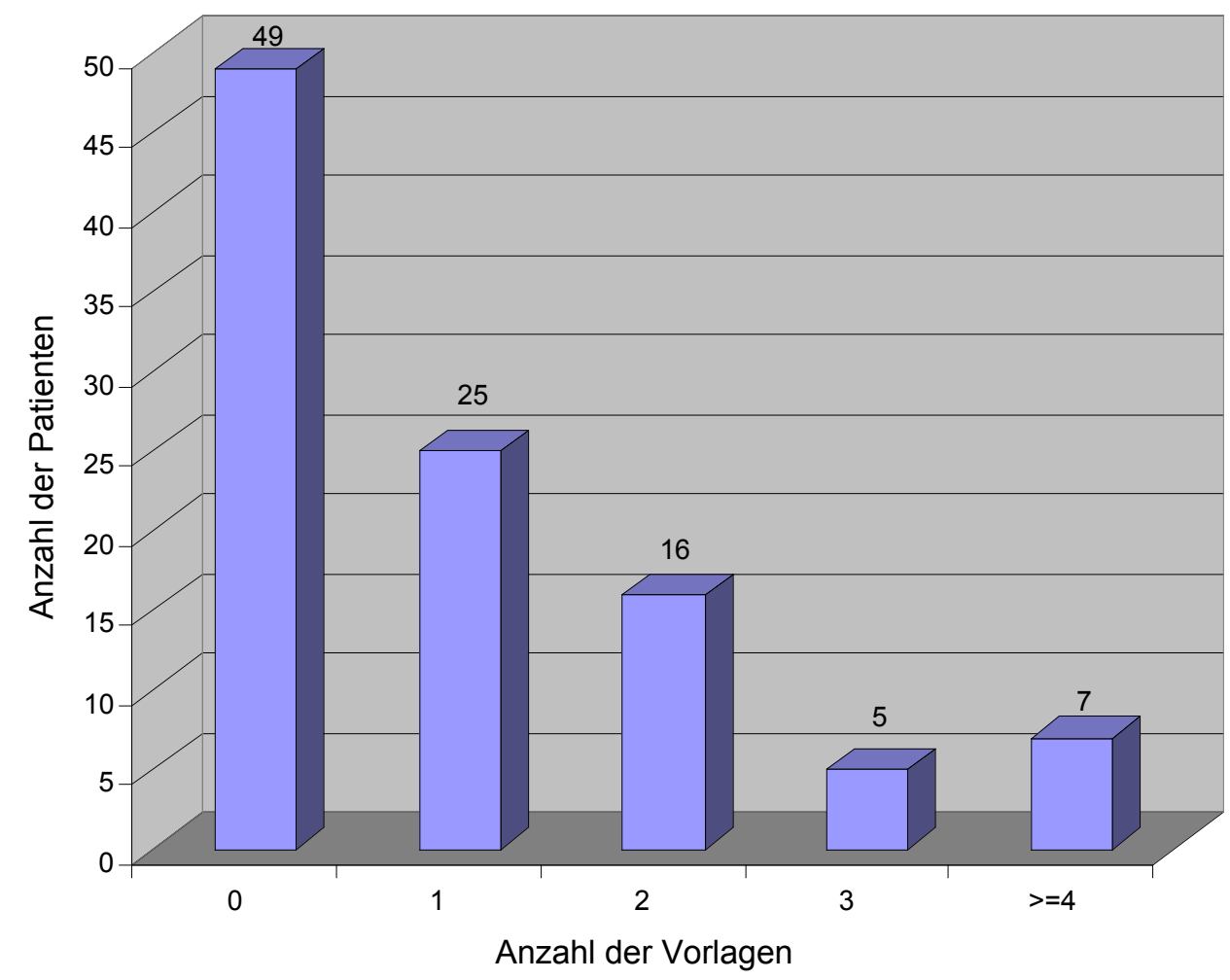

Abb.36: Übersicht über Verteilung und Anzahl der Vorlagen

Von den 102 vorhandenen Antworten zu dieser Frage geben 49 Befragte an, keine Vorlage zu benutzen. Eine Vorlage täglich benötigen 25 Patienten. 16 Operierte brauchen 2, 5 Patienten 3 Vorlagen. 4 oder mehr Vorlagen benötigen 7 Patienten (Abb.36). 
Die Frage nach der Häufigkeit des Harnverlustes ergab folgendes Ergebnis.

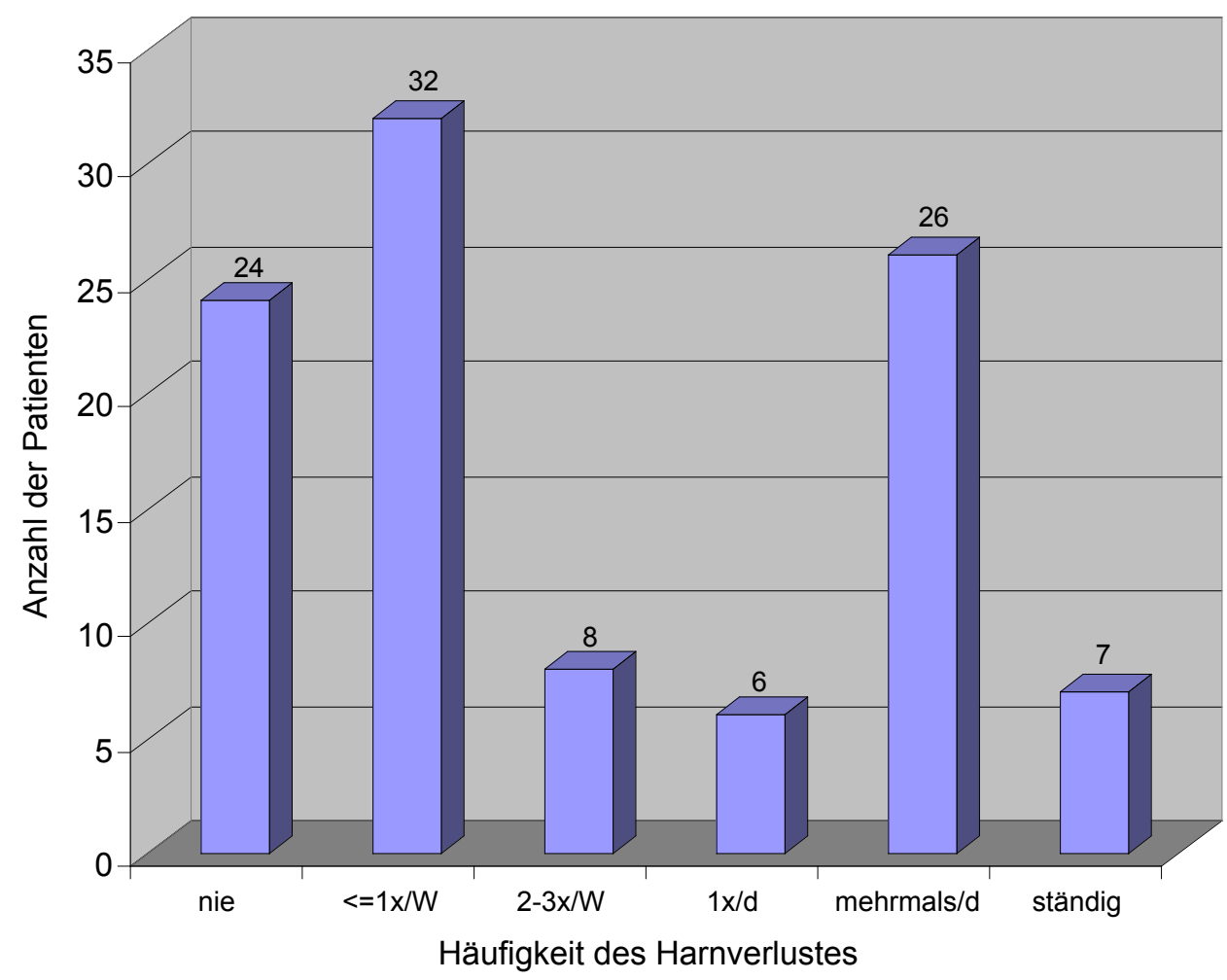

Abb.37: Übersicht über die Häufigkeit des Harnverlustes postoperativ

Keinen Harnverlust gaben 24 Patienten an. Während 32 der Befragten höchstens einmal pro Woche unkontrollierten Harnverlust hatten, traf dies bei 8 Patienten 23 mal pro Woche, bei 6 Patienten einmal täglich und bei 26 Patienten mehrmals täglich zu. Ständigen Harnverlust gaben 7 Patienten an (Abb.37).

Des Weiteren wurde erfragt, ob es im postoperativen Verlauf zu einer Verbesserung des Kontinenzstatus kam, und wenn ja, wann sich der Kontinenzstatus zum Zeitpunkt der Befragung eingestellt hat. Bei 33 Patienten (31,4\%) hat sich die direkte postoperative Situation nicht geändert. 72 der Befragten $(68,6 \%)$ gaben eine Verbesserung ihres Kontinenzstatus nach durchschnittlich 6,9 Monaten an, wobei die Zeitspanne von einem Monat zu höchstens 40 Monaten reichte.

Von diesen 72 Patienten haben 13 (12,4\% vom Gesamtkollektiv) direkt postoperativ eine Inkontinenz angegeben, haben aber in einer durchschnittlichen Zeit von 
4,5 Monaten eine absolute Kontinenz (Grad 0) erreicht. Hierbei reichte die Zeitspanne von mindestens einem Monat bis zu 12 Monaten.

\subsubsection{Lebensqualität im Bezug auf den Kontinenzstatus}

In einer Skala von 0 bis $10(0=$ nicht beeinflusst, $10=$ stark beeinflusst $)$ konnten die Patienten die Beeinflussung eines evtl. Harnverlustes auf ihren Lebensalltag einschätzen. Mit Ausnahme der 5 Patienten, die vor der Operation eine Inkontinenz angegeben hatten, haben die restlichen 99 Patienten sich präoperativ nicht beeinflusst gefühlt.

Postoperativ behauptet dies nur noch etwa ein Drittel der Patienten. Ein weiteres Drittel fühlt sich wenig beeinträchtigt, während das letzte Drittel sich mittel- bis schwergradig belastet fühlt (Abb.38).

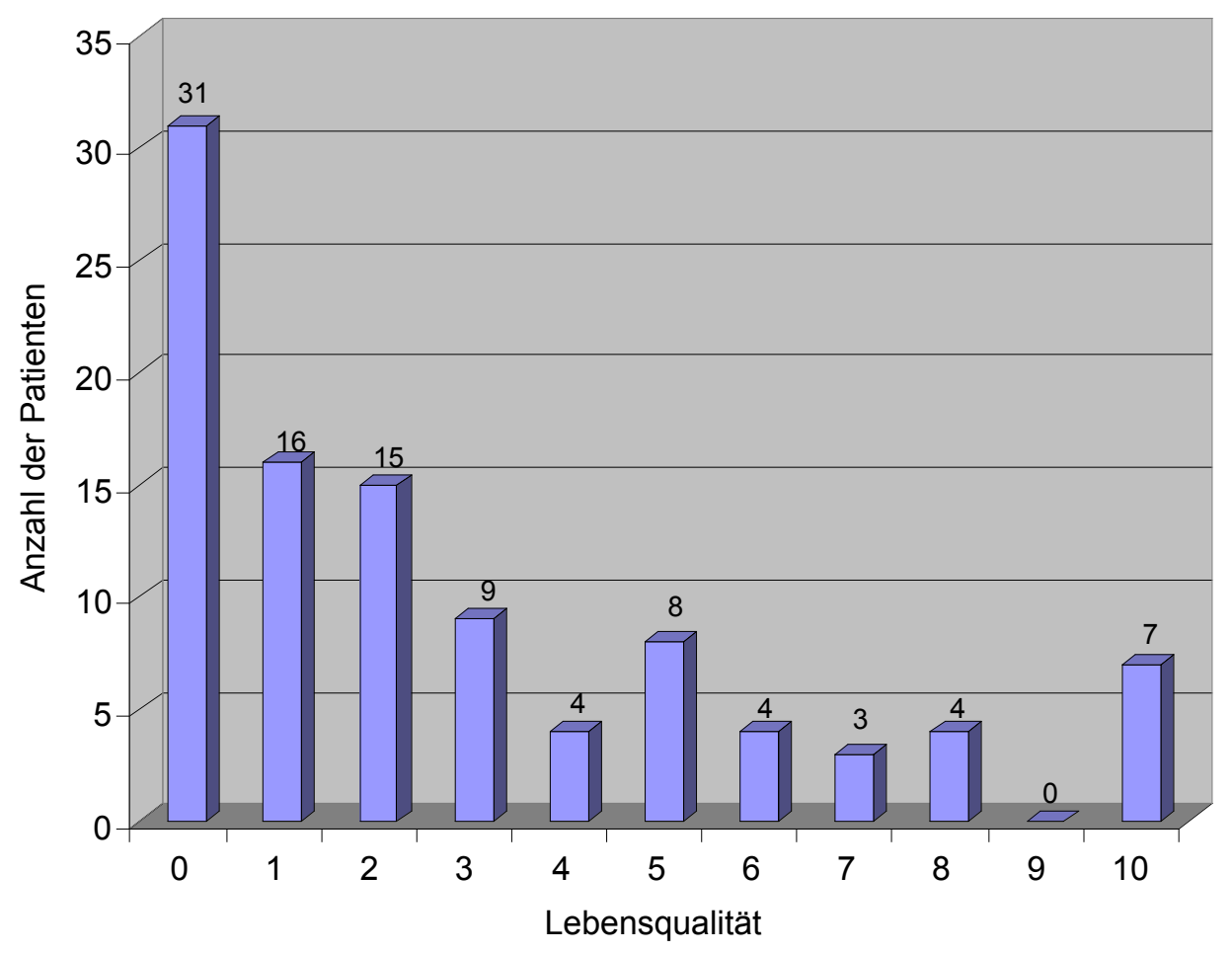

Abb.38: Postoperative Selbsteinschätzung der Beeinflussung des Lebens durch Harnverlust $(0=$ nicht beeinflusst, $10=$ stark beeinflusst $)$ 


\subsubsection{Weitere die Kontinenz beeinflussende Faktoren}

\subsubsection{Alter}

Zur genaueren Differenzierung der Ergebnisse zum Kontinenzstatus wurden die Patienten in eine Gruppe $<=59$ Jahre, in eine mittlere Altersgruppe $>=60$ bis $<=69$ und eine weitere Gruppe $>=70$ Jahre eingeteilt und diese wurden miteinander verglichen (Abb.39).

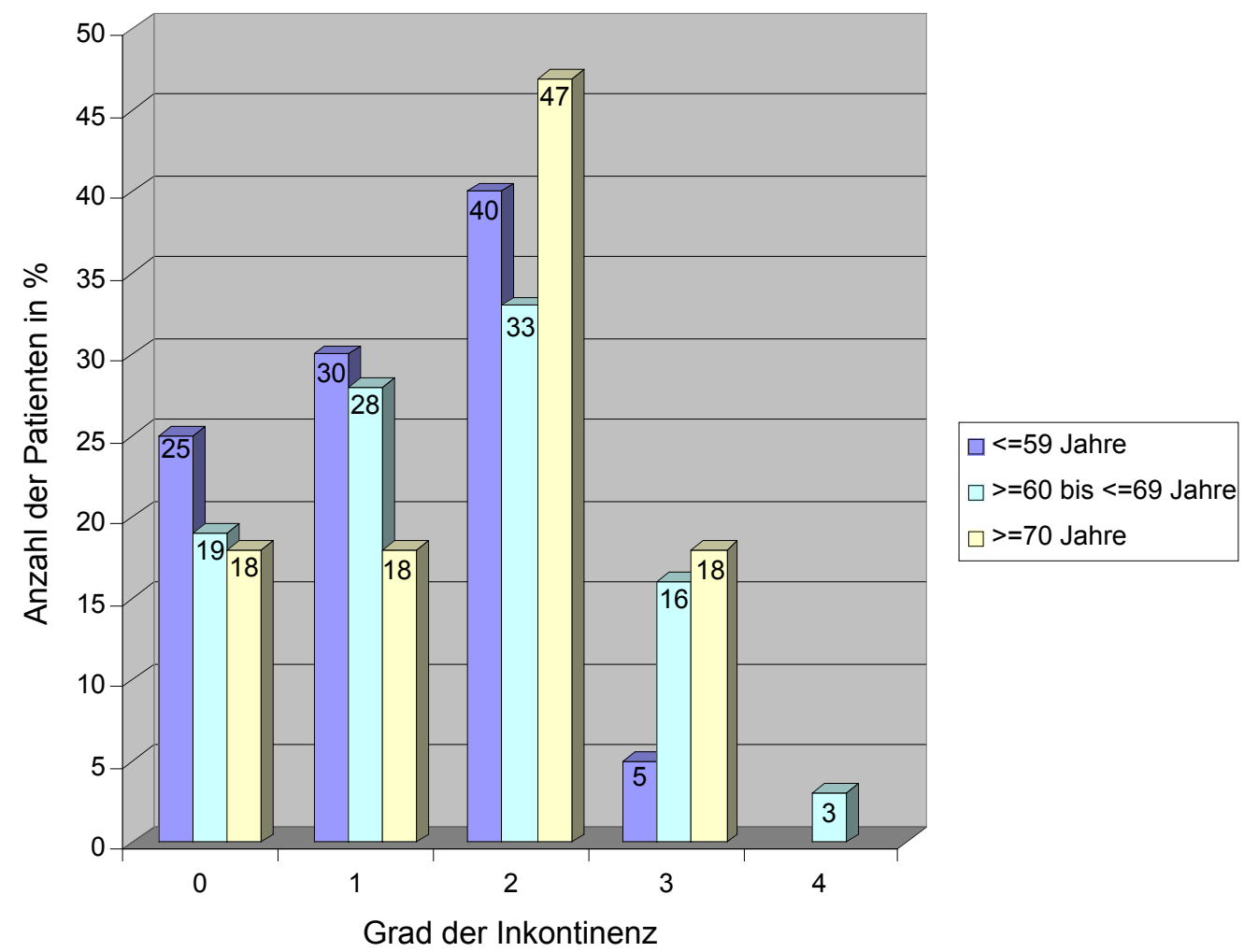

Abb.39: Vergleich des Kontinenzstatus der Altersgruppen

Bei einem Vergleich der drei Gruppen durch den Kruskal-Wallis-Test wurde ein $p=0,43$ errechnet. Es ist kein statistisch signifikanter Unterschied feststellbar. Das Alter hat statistisch bei unseren Patienten keinen Einfluss auf den Kontinenzstatus. 


\subsubsection{Nervenschonende Operation}

Das Gesamtkollektiv der Patienten wurde in eine Gruppe, die nervenschonend operiert werden konnte und in eine zweite Gruppe, bei denen das neurovaskuläre Bündel nicht geschont werden konnte, geteilt (Abb.40).

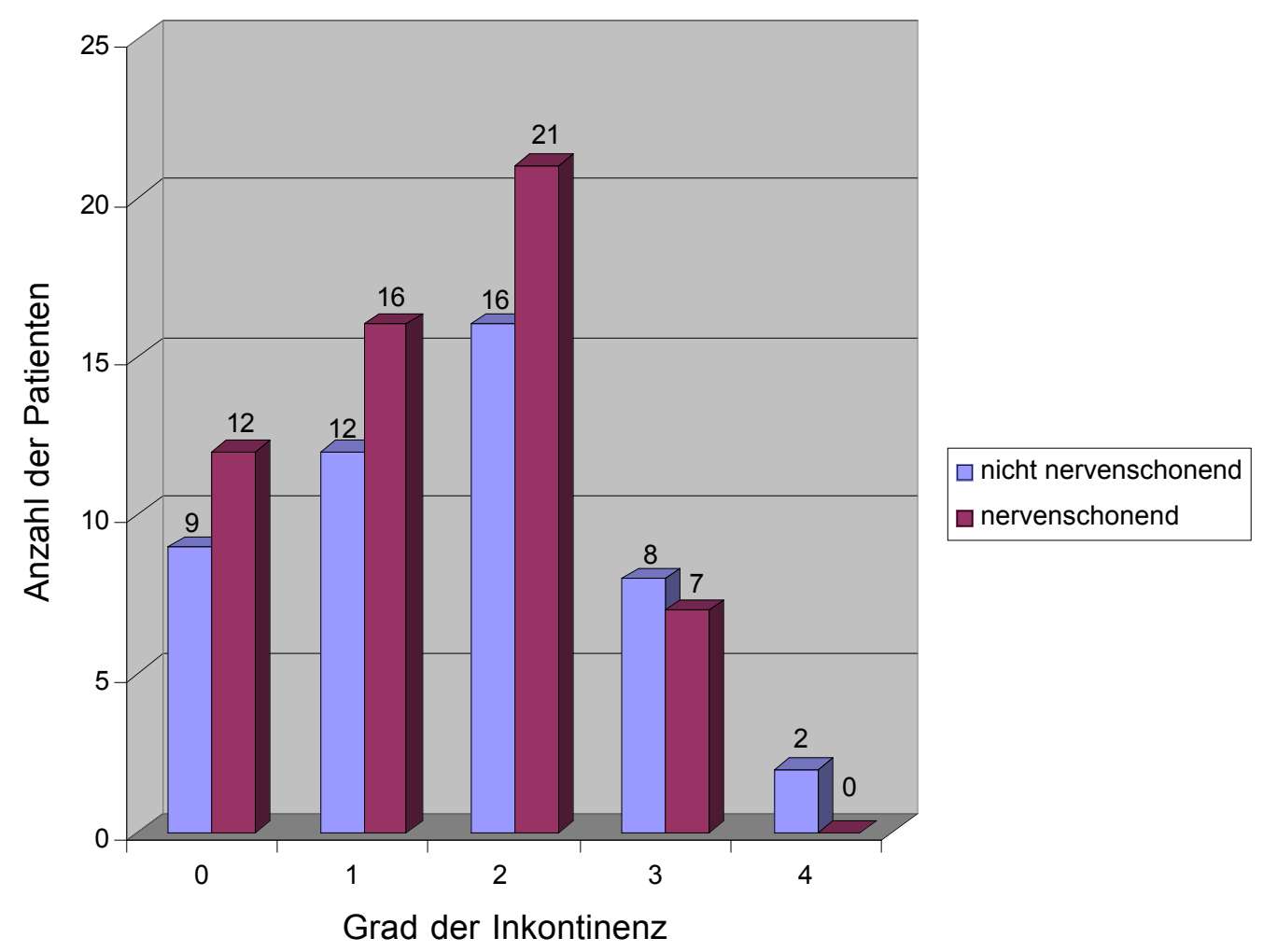

Abb.40: Vergleich des Kontinenzstatus der nervenschonend Operierten mit dem der nicht-nervenschonend operierten Patienten

Vergleicht man diese beiden Gruppen hinsichtlich des Kontinenzstatus nach dem operativen Eingriff, so zeigt es sich, dass kein signifikanter Unterschied feststellbar ist (Mann-Whitney-U-Test mit $p=0,32$ ).

\subsubsection{Potenzstatus}

Mittels des validierten „Fragebogens zur sexuellen Gesundheit des Mannes“ (IIEF-5) wurde zunächst der Status zum Zeitpunkt vor der Operation erhoben. Es zeigt sich, dass 69 Patienten (67\%) einen Score $>22$ erreicht haben. Dies entspricht nach IIEF-5 keinen Anzeichen einer erektilen Dysfunktion. Fraglich ist die sexuelle Funktion bei 29 der Befragten (28\%), bei denen ein Score zwischen acht 
und 22 errechnet wurde. Eine erektile Dysfunktion ist bei 5 Patienten (5\%) vor der Operation festzustellen. Die genaue Verteilung ist in der Abbildung 41 dargestellt.

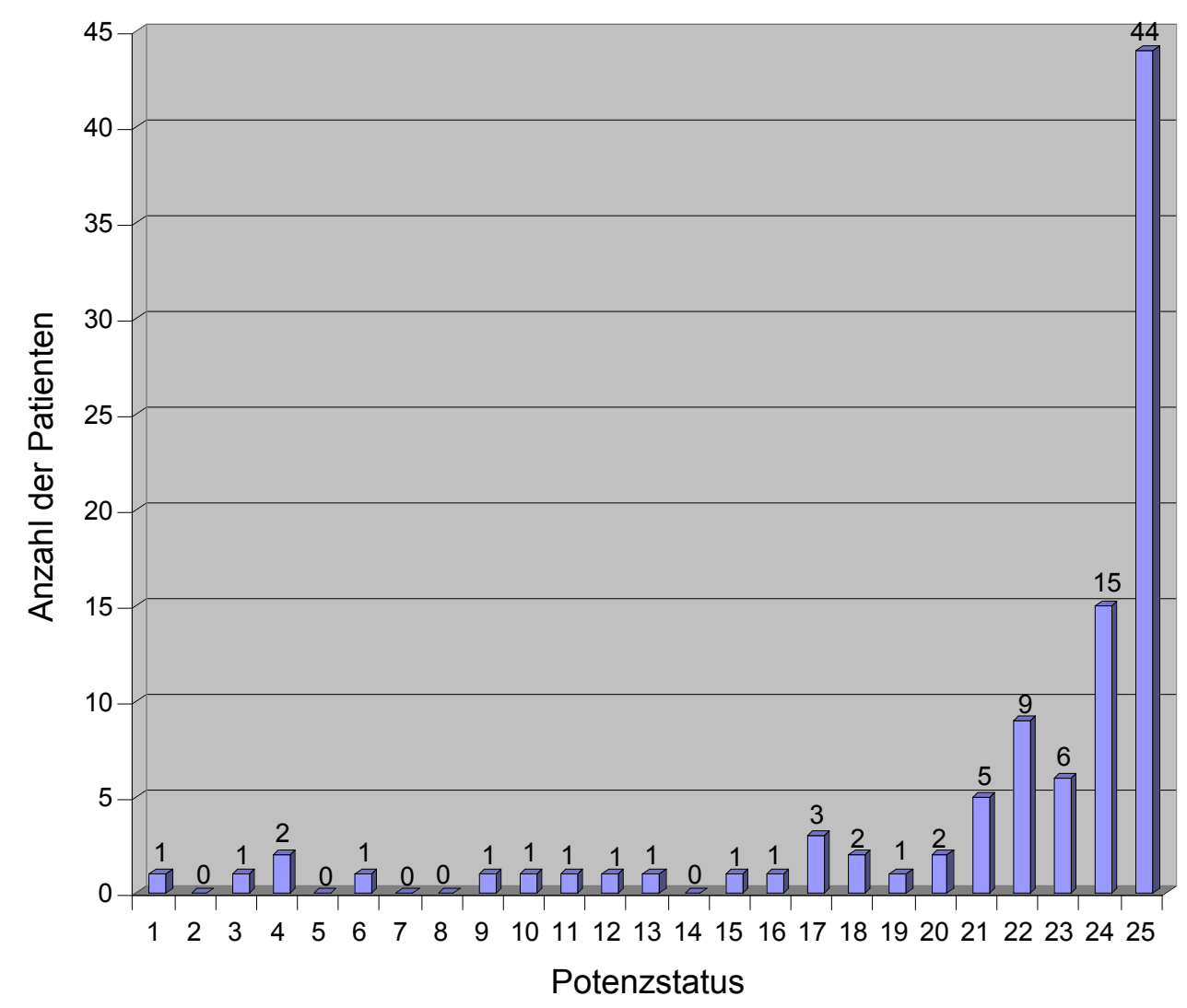

Abb.41: Potenzstatus der Patienten vor der Operation

Der Potenzstatus zum Zeitpunkt der Befragung, also nach der radikalen Prostatektomie, stellt sich wie folgt dar: bei 90 Patienten (86,5\%) wurde ein Score $<=5$ durch den IIEF-5 ermittelt. Es ist von einer stark eingeschränkten sexuellen Funktion auszugehen. Bei den restlichen 14 Operierten (13,5\%) wurde ein Score zwischen 6 und 20 ermittelt. Hier ist eine erektile Dysfunktion fraglich. Die exakte Verteilung ist in Abbildung 42 dargestellt. 


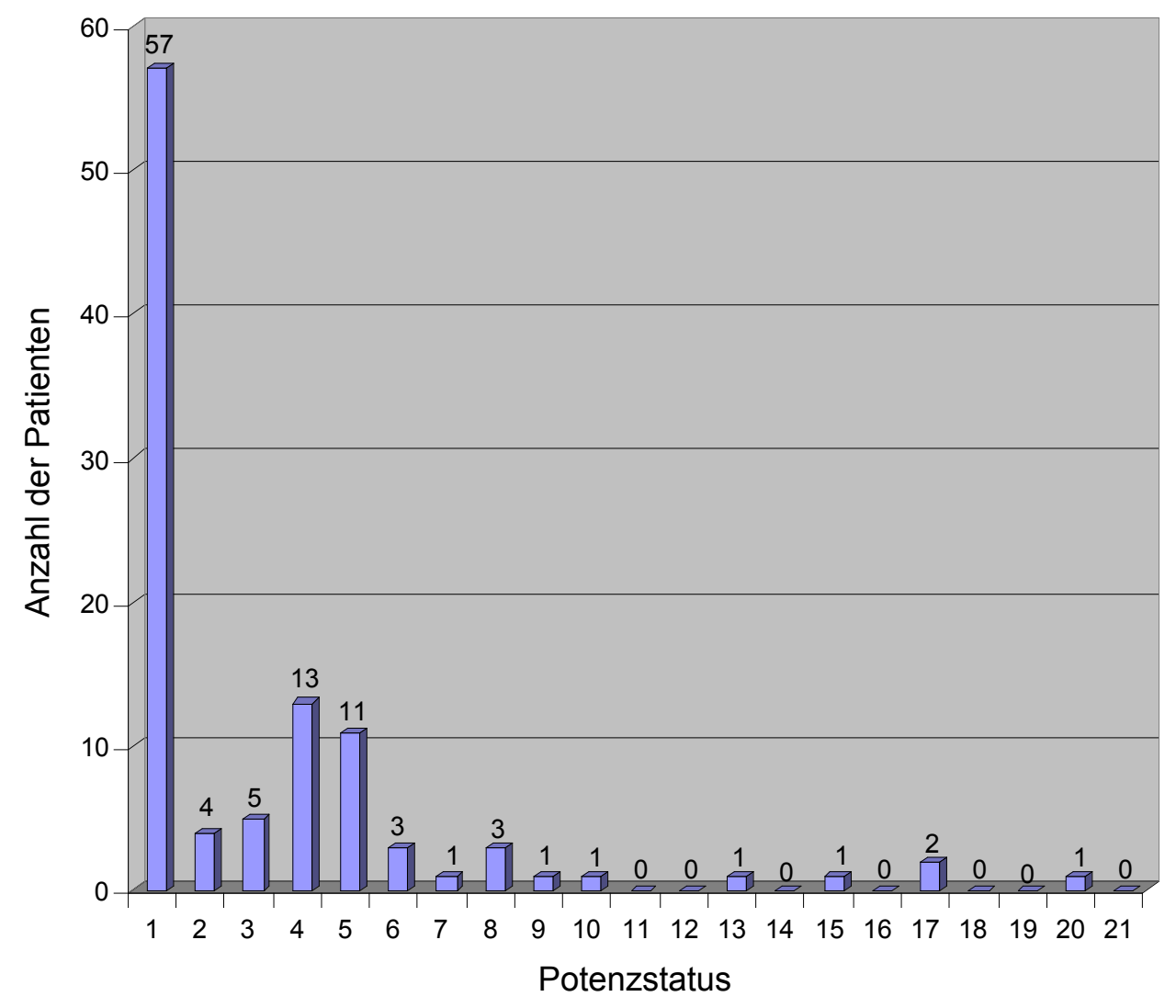

Abb.42: Potenzstatus der Patienten nach der Operation

Des Weiteren wurde erfragt, ob es im postoperativen Verlauf zu einer Verbesserung des Potenzstatus kam, und wenn ja, wann sich der Potenzstatus zum Zeitpunkt der Befragung eingestellt hat. Bei 4 Patienten (4\%) hat sich die direkte postoperative Situation geändert. Bei 2 dieser Patienten trat die Verbesserung nach 6 Monaten auf, bei einem nach 10 Monaten und ein weiterer Befragter gab eine Verbesserung nach 12 Monaten an.

\subsubsection{Nervenschonende Operation}

Vergleicht man diejenigen Patienten, bei denen intraoperativ das Nervenbündel zur Möglichkeit der Erhaltung der Errektionsfähigkeit geschont wurde, mit denjenigen, bei denen dies nicht möglich war, so zeigt sich die in der Abbildung 43 aufgeführte Verteilung. Es ist statistisch kein signifikanter Unterschied feststellbar. 


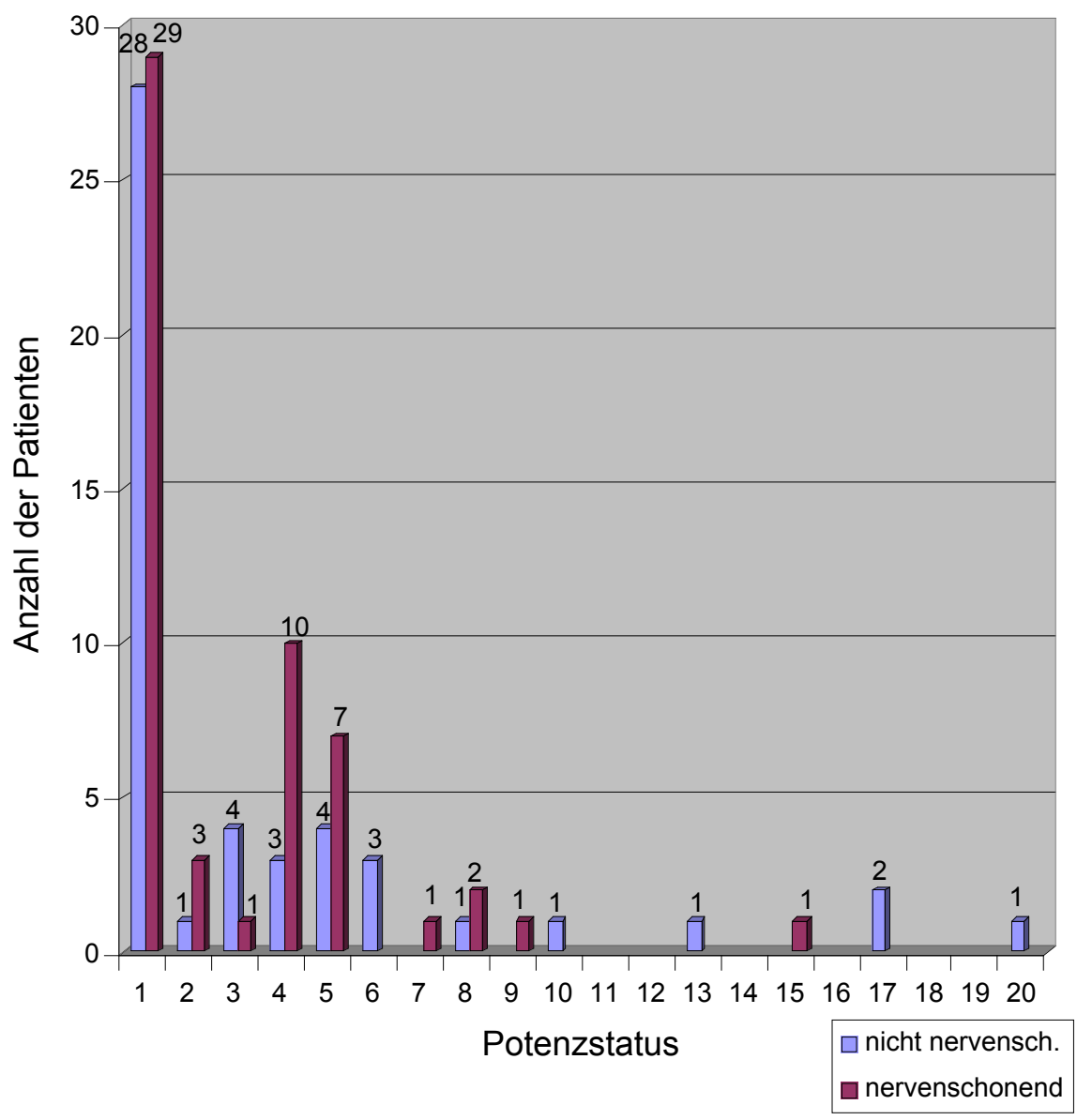

Abb.43: Vergleich des Potenzstatus der nervenschonend gegenüber den nicht nervenschonend operierten Patienten 


\section{Diskussion}

\subsection{Zielsetzung}

Ziel der Arbeit ist es zu zeigen, ob die Ausbreitung des Prostatakarzinoms mit Hilfe der diagnostischen Möglichkeiten wie der Bestimmung des präoperativen PSA-Wertes und der histopathologischen Untersuchung der aus dem Organ entnommenen Stanzbiopsien festzustellen und ein passendes Therapiekonzept auf dieser Basis festzulegen ist.

Weiterhin sollen klinische Aspekte, wie die postoperative Kontinenz, die postoperative Erektionsfähigkeit, insbesondere im Hinblick auf den Erfolg des nervenschonenden Operationsverfahrens, sowie die Auswirkungen eines solchen Eingriffes auf die Lebensqualität der Patienten beleuchtet werden.

\subsection{Methode}

Die Auswahl des Patientenkollektivs wurde auf alle in einem Zeitraum von 4 Jahren im Uniklinikum Göttingen prostatektomierten Patienten begrenzt. Hier findet sich eine für statistische Zwecke valide Anzahl von 142 Operierten. Die Daten wurden retrospektiv erhoben. Zum einen wurden sie der Dokumentation in den Krankenakten entnommen. Die so gewonnenen Ergebnisse beruhen also auf Fakten und sind sehr zuverlässig. Zum anderen wurden einige Daten mittels eines Fragebogens mit zum großen Teil validierten Fragen erhoben. Hier spielt die subjektive Einschätzung eines jeden Patienten eine Rolle. Es lässt sich aber festhalten, dass durch die relativ hohe Teilnahme der Patienten $(73,3 \%)$ und den hohen Anteil an validierten Fragen auch hier eine hohe Wahrscheinlichkeit der Genauigkeit der erhobenen Daten gegeben ist. Somit lässt sich feststellen, dass die Ergebnisse auf sehr validen Daten basieren und somit eine hohe Gültigkeit besitzen. 


\subsection{Histologie der Stanzen und Prostatapräparate}

Entscheidungen zu Therapiekonzepten für das lokal begrenzte Prostatakarzinom basieren neben dem PSA-Wert weitestgehend auf Informationen aus der Biopsie der Prostata. Bei unserem Patientenkollektiv wurde auf Grundlage dieser Informationen bei jedem Patienten eine radikale retropubische Protatektomie durchgeführt.

Diese Informationen aus den Stanzzylindern der Biopsie sind nur nützlich für Therapieentscheidungen, wenn sie mit den Ergebnissen der histologischen Untersuchung des Prostatapräparats nach der Operation weitestgehend korrelieren (Obek et al. 1999). Im folgenden gilt es die Ergebnisse der vorliegenden retrospektiven Studie im Hinblick darauf zu diskutieren, welche Informationen aus den Biopsien im oben genannten Sinne nützlich sind für die weiteren Therapieentscheidungen. Die zu untersuchenden Parameter reichen von grundsätzlichen Informationen, wie dem Nachweis von einem Karzinom, über die Ausbreitung auf die Seitenlappen der Prostata (uni- bzw. bilateraler Tumorbefall) hin zu Einteilungen der Biopsien nach Gleason und dem Grading-System.

Der Nachweis eines Karzinoms konnte präoperativ mittels Gewebeproben bei allen Patienten erbracht werden und war Voraussetzung für die radikale Prostatektomie. Im postoperativen Präparat konnte bei 137 Patienten (97,9\%) eine maligne Neoplasie bestätigt werden. Bei 3 Patienten (2,1\%) konnte kein Karzinom mehr nachgewiesen werden.

Der vermutliche Grund für dieses Ergebnis findet sich in der Literatur. Goldstein et al. führten den Begriff des ,,vanishing cancer phenomenon“ ein (Goldstein et al. 1995). Hier wird über 13 Fälle berichtet, bei denen nach positiver Biopsie in dem postoperativen Prostatapräparat kein Karzinom nachgewiesen werden konnte. Die in der Literatur beschriebene Inzidenz hierfür reicht von 0,07 bis 4,2 \% der Fälle. Es gibt verschiedene Gründe hierfür. In einigen Fällen wurde das betroffene Gewebe durch die Stanzen entfernt. Teilweise wurden falsch positive Befunde der Biopsien oder falsch negative Befunde bei der Beurteilung des Prostatapräparates durch den Pathologen erhoben (Bostwick und Bostwick 2004, Goldstein et al. 
1995, Herkommer et al. 2004, Srigley 2003, Trpkov et al. 2006, Zynger et al. 2006).

Unser Ergebnis deckt sich mit 2,1\% der Fälle mit den Angaben in der aktuellen wissenschaftlichen Literatur.

Im Folgenden sollen die Ergebnisse aus dem Vergleich der Biopsien mit den Befunden der postoperativen pathologischen Untersuchung in Bezug auf den unioder bilateralen Befall der Prostata diskutiert werden. Der uni- bzw. bilaterale Seitenlappenbefall der Prostatadrüse ist Grundlage für wichtige Therapieentscheidungen. Beispielsweise wurde auf der Grundlage eines unilateralen Befalls der Prostata eine nervenschonende Operation zur möglichen Potenzerhaltung erwogen.

Die Auswertung der Patientendaten zeigt hinsichtlich der präoperativen Histologien einen uni- bzw. bilateralen Seitenlappenbefall bei 61,4 bzw. 38,6 \% der Patienten. Postoperativ unterscheidet sich der uni- bzw. bilaterale Befall der Prostata mit 33,8 bzw. 66,2 \% der Patienten signifikant von dem präoperativen Ergebnis. Wie lässt sich diese Verschiebung zugunsten des bilateralen Befalls erklären?

Betrachtet man die Patientengruppe, bei der postoperativ ein bilateraler Seitenlappenbefall dokumentiert wurde, so fällt auf, dass bei 43,8 \% der Patienten der Tumorbefall präoperativ nur in einem Seitenlappen diagnostiziert wurde. Hier spiegelt sich die Schwierigkeit des aktuellen Biopsieverfahrens wider. Zum Teil wird das maligne Gewebe bei den Stanzen nicht getroffen, unterstützende Bildgebung ist teilweise noch sehr ungenau, die Biopsien wurden bei unseren Patienten durch verschiedene Ärzte vorgenommen und nicht zuletzt ist das Biopsieverfahren nicht standardisiert. Hierdurch lässt sich das veränderte postoperative Ergebnis zugunsten des bilateralen Seitenlappenbefalls nachvollziehen. Dass bei den Patienten mit postoperativ gesichertem unilateralem Befall der Prostata sich 4 Patienten fanden, bei denen durch die Biopsie ein bilateraler Befall dokumentiert wurde, relativiert das Ergebnis ein wenig.

Betrachtet man die Patientengruppe, bei der präoperativ ein unilateraler Befall festgestellt wurde, so zeigt es sich, dass bei $47,7 \%$ der Patienten auch in dem 
kontralateralen Seitenlappen postoperativ am Präparat ein Karzinom festgestellt wurde. Bei diesen 32 Patienten wurde in dem Seitenlappen, der vor der Operation durch die Stanze für gutartig erklärt wurde, im Nachhinein eine maligne Neoplasie nachgewiesen.

In der aktuell zur Verfügung stehenden Literatur haben nur wenige Studien die Korrelation von präoperativen Biopsieparametern mit den postoperativen pathologischen Ergebnissen verglichen (unilateraler gegen bilateraler Befall der Prostataseitenlappen).

Obek und Mitarbeiter untersuchten 155 Patienten, die wie unser Patientenkollektiv radikal retropubisch prostatektomiert wurden. Die präoperativen Histologien zeigten einen uni- bzw. bilateralen Seitenlappenbefall bei 64,5 bzw. 35,5\% der Patienten. Postoperativ verschiebt sich auch hier die Verteilung mit 26 bzw. 74 \% zugunsten des bilateralen Befalls. Bei den 100 Patienten, die eine unilateral negative Biopsie hatten, fand sich bei 74 im postoperativen Präparat ein bilateraler Befall. Schlussfolgernd stellen Obek et al. fest, dass die Interpretation der Prostatabiopsie kein exaktes Hilfsmittel für die Ausbreitung des Prostatakarzinoms ist (Obek et al. 1999). Weitere wissenschaftliche Arbeiten bestätigen diese Ergebnisse. Connolly und Mitarbeiter ( $n=133$; präoperativ unilateral $75 / 133$, postoperativ bilateral 113/133) schlussfolgern, dass ein durch die Biopsie diagnostiziertes unilaterales Karzinom mit einer hohen Inzidenz in der endgültigen postoperativen Histologie mit einem bilateralen Befall assoziiert ist (Connolly et al. 2004). Scales und seine Gruppe halten fest, dass Therapiekonzepte, die auf Grundlage von unilateralem Tumorbefall der Prostata basieren, für den hohen Prozentsatz der falsch unilateral diagnostizierten Patienten mit großer Wahrscheinlichkeit nicht kurativ sind (Scales et al. 2007). Die Augsburger Gruppe um Weckermann stellt fest, dass es nicht möglich ist, zuverlässig auf Basis der Biopsie einen unilateralen Befall der Prostata vorauszusagen (Weckermann et al. 2007).

Unsere Zahlen stimmen mit denen in der Literatur veröffentlichten weitgehend überein. Eine Therapieentscheidung auf Grundlage des uni- oder bilateralen Sei- 
tenlappenbefalls der Prostata beruht auf sehr ungenauen Daten und ist deshalb nicht ratsam.

Bis vor einigen Jahren war in der Diagnostik des lokalen Prostatakarzinoms die von Hodge und Mitarbeitern beschriebene Technik der Sextanten-Biopsie der Gold-Standard (Hodge et al. 1989, Salomon et al. 1997). In den letzten 10 Jahren wurden viele Biopsie-Protokolle entwickelt. Einzelne Protokolle wurden etabliert, welche die Anzahl der Stanzzylinder angehoben haben, indem die Sextantenbiopsie um weitere laterale Biopsien erweitert wurde. Hierdurch sollte die Karzinomerkennung, sowie dessen Ausbreitung und Aggressivität besser diagnostiziert werden (Djavan und Margreiter 2007).

Konnte eine Erweiterung der Biopsie die Erkennung des Prostatakarzinoms bei unseren Patienten verbessern?

Um diese Frage zu beantworten, wurden unsere Ergebnisse der Biopsie der Patienten, bei denen 6 bzw. 10 oder mehr Stanzen entnommen wurden, miteinander verglichen. Betrachtet man diese beiden Patientengruppen im Bezug auf den unibzw. bilateralen Seitenlappenbefall, so war der präoperative Befund in $70 \mathrm{bzw}$. $61 \%$ mit dem postoperativen identisch. „Undergraded“ waren 28 bzw. 26\%, „overgraded“ 2 bzw. 13\%. Es konnte statistisch kein signifikanter Unterschied festgestellt werden.

Vergleicht man die Patientengruppen hinsichtlich des Gleason-Scores, bei denen 6 bzw. 10 oder mehr Stanzen entnommen wurden, so ist deutlich die Tendenz zu erkennen, dass bei 10 und mehr entnommen Biopsien häufiger Übereinstimmungen dokumentiert sind. So finden sich in dieser Gruppe 33,3\% Übereinstimmungen, während dies bei der Gruppe mit 6 Stanzen nur auf 25,5\% zutrifft. Einschränkend muss erwähnt werden, dass die Fallzahl mit n=39 (6 Stanzen) bzw. $\mathrm{n}=21(>=10$ Stanzen $)$ sehr niedrig ist.

In der aktuellen wissenschaftlichen Literatur finden sich zu dieser Fragestellung fast ausschließlich Studien, welche die Erweiterung der Sextanten-Biopsie empfehlen. So schreiben Paul und Mitarbeiter, dass der Vorteil durch zusätzliche Biopsien gering sei, aber statistisch signifikant. Da kein Unterschied bei der Morbidität und eine hohe Patientenakzeptanz festzustellen wäre, sei die 10-Stanzen Bi- 
opsie zu empfehlen (Paul et al. 2005). Andere Gruppen haben noch deutlichere Ergebnisse publiziert. Die 10-Stanzen-Biopsie-Technik sei besser bei der Erkennung von Prostatakarzinomen als die übliche Sextanten-Biopsie-Technik und deshalb zur Diagnostik des Prostatakarzinoms empfohlen (Eskicorapci et al. 2004, Fink et al. 2003, Gore et al. 2001, Matsubara et al. 2006). Wissenschaftliche Publikationen, die weiterhin Sextanten-Biopsie empfehlen sind kaum zu finden. Grossklaus und Mitarbeiter stellten fest, dass eine Erhöhung der Stanzbiopsien über die übliche Sextantenbiopsie hinaus keine Verbesserung der prognostischen Informationen bringe. Es gäbe kein Unterschied bei der Anzahl der positiven Stanzen oder bilateral positiven Stanzen zwischen Patienten mit mehr oder weniger als 6 Biopsien (Grossklaus et al. 2001).

Bei dieser Untersuchung beruhen unsere Daten auf einer geringen Anzahl von Patienten. Die Biopsien erfolgten nicht nach einem standardisierten Verfahren und wurden durch verschiedene Ärzte entnommen. Hinsichtlich des Seitenlappenbefalls konnte keine Verbesserung der Genauigkeit der aus den Gewebeproben gewonnen Daten festgestellt werden, wenn die Anzahl der Stanzen auf 10 oder mehr angehoben wurde. Hinsichtlich des Gleason-Scores ist eine Tendenz zur Verbesserung durch eine höhere Probenanzahl zu erkennen. Dies ist aus oben genannten Gründen nur als Tendenz zu werten. Genauere Untersuchungen zu dieser Fragestellung auf Grundlage standardisierter Biopsieverfahren und einer größeren Patientenanzahl ließen mit hoher Wahrscheinlichkeit exaktere Aussagen zu.

Ist abgesehen von den üblichen Verfahren der Sextanten- bzw. der 10-StanzenBiopsie-Technik ein Unterschied bei der Erkennung des Prostatakarzinoms anhand der Menge der entnommenen Stanzen zu erkennen?

Betrachtet man die Ergebnisse der Fälle, bei denen postoperativ definitiv ein unilateraler Befall der Prostata festgestellt wurde, so fällt auf, dass mit steigender Anzahl der Stanzen die Sensitivität dieser Untersuchung abnimmt. Bei 1-5 entnommenen Stanzen stimmte die Vorhersage vom unilateralen Befall der Prostata zu 100\% mit dem postoperativen Ergebnis überein. Bei 6-10 entnommenen Stanzen wurde postoperativ bei $11,5 \%$ der Fälle ein bilateraler Befall der Prostata festgestellt. Diese Tendenz nimmt zu, wenn man die Patienten betrachtet, bei de- 
nen 11 oder mehr Stanzen entnommen wurden. Hier wurde in $50 \%$ der Fälle eine bilateraler Befall belegt.

Wie kommt ein solches Ergebnis zustande? Zunächst muss erwähnt werden, dass mit $n=39$ die Fallzahl für diese Untersuchung nicht sehr hoch ist, besonders bei $>=11$ Stanzen fanden sich nur 2 Patienten. Aber eine Tendenz ist eindeutig erkennbar. Beeinflusst ist das Ergebnis sicherlich durch die nicht standardisierte Probenentnahme. Hier kann es bei der Biopsie durch den Untersucher auch zu Verwechslungen oder ungenaue Zuordnung der Proben zu den Seitenlappen kommen. Zudem fand vermutlich eine Vorselektion der Patienten statt. Patienten mit niedrigem PSA, unauffälliger digitaler rektaler Untersuchung und dem klinischen Verdacht auf ein low-grade Prostatakarzinom wurden evtl. weniger Stanzen entnommen, aufgrund der Wahrscheinlichkeit, dass nur ein unilateraler Befall der Prostata besteht. Das obig beschriebene „vanishing cancer phenomenon“ wird ebenfalls das Ergebnis beeinflusst haben.

Betrachtet man die Ergebnisse der Fälle, bei denen postoperativ ein bilateraler Befall der Prostata festgestellt wurde, so fällt auf, dass mit der steigenden Anzahl der Stanzen auch die Sensitivität für die Erkennung des Karzinoms steigt. Hier muss man davon ausgehen, dass ein bilateraler Tumorbefall der Prostata mit einem höheren Stadium einhergeht. Je mehr Stanzen entnommen werden, umso wahrscheinlicher ist es, dass das betroffene Gewebe bei Biopsie getroffen wird.

Hier ist mit Zunahme der Stanzenmenge eine Tendenz zu höherer Sensitivität erkennbar.

Die Untersuchung der Genauigkeit der Stanzen in Abhängigkeit von der Menge der entnommenen Stanzzylinder zeigte bei unseren Patienten keine signifikanten Unterschiede.

\subsection{Positiver Absetzungsrand (Radikalität)}

Bei unserem Patientenkollektiv fand sich bei 17,5\% ein positiver Absetzungsrand. Entsprechende Zahlen aus der aktuellen Literatur reichen von 12,8 \% bis 29\% R1- 
Resektionen bei der radikalen retropubischen Prostatektomie (Han et al. 2004, Swindle et al. 2005).

In einer Studie von Sofer et al. mit 734 Patienten, die sich einer radikalen retropubischen Prostatektomie unterzogen, belief sich der Anteil der R1-Befunde auf 29\% (Sofer et al. 2002). In einer weiteren großen Studie von Hull und Mitarbeitern mit 1000 Patienten betrug die Rate der positiven Absetzungsränder 12,8\% (Hull et al. 2002).

Unsere Ergebnisse sind mit denen der aktuellen wissenschaftlichen Literatur vergleichbar.

\subsection{Grading}

Aus den bei der Biopsie entnommenen Stanzen wird der histologische Differenzierungsgrad (Grading) bestimmt. Der Differenzierungsgrad wird als Ausmaß der Malignität des Tumors angesehen und ist wichtig für die Entscheidung zu Therapiemaßnahmen. Ist die Vorrausage des Malignitätsgrades möglich? Darf man diesen Parameter bei der Entscheidung zur Festlegung eines Therapieregimes berücksichtigen?

Bei unseren Patienten fanden sich präoperativ 20,7\% der Patienten mit dem Malignitätsgrad 1, bei $66,9 \%$ Grad 2 und bei 12,4\% eine undifferenzierte Anaplasie Grad 3. Postoperativ fanden sich 7,3\% der Patienten mit G1, 64,2 und 28,5\% mit dem Malignitätsgrad G2 bzw. G3.

Diese Ergebnisse sind im Wesentlichen mit den aktuellen Studien vergleichbar. So publizierten Szymanowski und Mitarbeiter zum präoperativen Grading in der G1-Gruppe 26,4\%, in der G2- bzw. G3-Gruppe 59,8 bzw. 13,8\% (Szymanowski et al. 2002). Bei Fischer et al. finden sich 27,7\% G1-Tumoren, 54,5 bzw. 17,7\% G2- bzw. G3-Tumoren (Fischer et al. 2005). Alschibaja und seine Mitarbeiter haben vergleichbar mit unserer Studie sowohl das prä- als auch den postoperativen Grading veröffentlicht. Hier fanden sich präoperativ 91\% G1- und G2Tumoren und 9\% G3-Tumoren. In der postoperativen Beurteilung verschieben sich die Werte wie bei unseren Patienten zu Gunsten der G3-Tumoren. Die Werte 
liegen bei 77\% G1- und G2-Tumoren sowie bei $23 \%$ der G3-Tumoren (Alschibaja et al. 2005).

Bei dem direkten Vergleich des prä- mit dem postoperativen Gradings bei unserem Patientenkollektiv zeigt sich eine Übereinstimmung der Ergebnisse in 63,3\% der Fälle. In 31,3\% der Fälle war die präoperative Beurteilung des Malignitätsgrades niedriger als postoperativ, in 5,5\% der Fälle verhielt es sich umgekehrt. Coogan und Mitarbeiter untersuchten hierzu 404 Patienten. Eine Übereinstimmung fand sich mit 48,3\% der Fälle bei etwas weniger Patienten als in unserer Studie. Undergrading wird in 35,4\% der Fälle beschrieben, was in etwa mit unserem Ergebnis übereinstimmt. Mit 16,3\% der Fälle beim Overgrading liegen Coogan et al. deutlich über dem Ergebnis unserer Patienten (Coogan et al. 2005).

Eine genaue Vorrausage des histologischen Differenzierungsgrades anhand der präoperativen Biopsie ist also nicht möglich. Aus diesem Grunde sollten auf Basis des Gradings keine Therapieentscheidungen getroffen werden.

\subsection{Prä- und postoperativer Gleason}

Sowohl bei der Diagnosestellung aus den Biopsien als auch aus dem ProstataPräparat wird der histologische Differenzierungsgrad nach Gleason bestimmt. Auch hier gilt es zu prüfen, wie Aussagekräftig die Bestimmung des GleasonScore aus den präoperativ entnommenen Stanzen ist. Denn auch hier gilt es, anhand des Gleason-Scores, möglichst genau die Charakteristik und Aggressivität des Tumors zu bestimmen, da dieses für den Patienten und den behandelnden Arzt für den Erfolg von möglichen Therapieregimen von überragender Bedeutung ist.

Vergleicht man bei unseren Patienten den prä- mit dem postoperativen GleasonScore im Einzelnen, so zeigt sich eindeutig eine Tendenz zum präoperativen Undergrading. So finden sich präoperativ bei Gleason 2-6 noch 75,7 \% der Fälle, postoperativ aber nur noch 53,7\%. Gegenläufig ist es bei den Fällen mit GleasonScore 7-10: präoperativ 24,3\% der Fälle, postoperativ 46,5\% (Tab.14). 


\begin{tabular}{|c|c|c|c|c|c|c|c|c|}
\hline \multirow[b]{2}{*}{$\begin{array}{c}\text { Gleason- } \\
\text { Score }\end{array}$} & \multicolumn{2}{|c|}{$\begin{array}{c}\text { Unsere Patien- } \\
\text { ten }\end{array}$} & \multicolumn{2}{|c|}{$\begin{array}{l}\text { Poulus et al. } \\
\qquad(2005)\end{array}$} & \multicolumn{2}{|c|}{$\begin{array}{l}\text { Egevad } \\
\text { (2001) }\end{array}$} & \multicolumn{2}{|c|}{$\begin{array}{l}\text { Lattouf und } \\
\text { Saad } \\
\text { (2002) }\end{array}$} \\
\hline & $\begin{array}{l}\text { prä. } \\
\text { n (\%) }\end{array}$ & $\begin{array}{l}\text { post. } \\
\text { n (\%) }\end{array}$ & $\begin{array}{l}\text { prä. } \\
\text { n (\%) }\end{array}$ & $\begin{array}{l}\text { post. } \\
\mathrm{n}(\%)\end{array}$ & $\begin{array}{l}\text { prä. } \\
\text { n (\%) }\end{array}$ & $\begin{array}{l}\text { post. } \\
\text { n (\%) }\end{array}$ & $\begin{array}{l}\text { prä. } \\
\text { n (\%) }\end{array}$ & $\begin{array}{l}\text { post. } \\
\mathrm{n}(\%)\end{array}$ \\
\hline $2-4$ & $23(20)$ & $9(7,9)$ & $2(1,3)$ & - & $4(3,3)$ & $4(3,3)$ & $70(18)$ & $66(17)$ \\
\hline $5-6$ & $\begin{array}{c}64 \\
(55,7)\end{array}$ & $\begin{array}{c}52 \\
(45,6)\end{array}$ & $68(45)$ & $\begin{array}{c}55 \\
(36,4)\end{array}$ & $\begin{array}{c}79 \\
(65,3)\end{array}$ & $\begin{array}{c}61 \\
(50,4)\end{array}$ & $\begin{array}{l}241 \\
(62)\end{array}$ & $\begin{array}{l}201 \\
(51)\end{array}$ \\
\hline 7 & $\begin{array}{c}16 \\
(13,9)\end{array}$ & $\begin{array}{c}26 \\
(22,8)\end{array}$ & $\begin{array}{c}64 \\
(42,4)\end{array}$ & $\begin{array}{c}78 \\
(51,7)\end{array}$ & $\begin{array}{c}27 \\
(22,3)\end{array}$ & $\begin{array}{c}35 \\
(28,9)\end{array}$ & $64(16)$ & $99(25)$ \\
\hline $8-10$ & $\begin{array}{c}12 \\
(10,4)\end{array}$ & $\begin{array}{c}27 \\
(23,7)\end{array}$ & $\begin{array}{c}17 \\
(11,3)\end{array}$ & $\begin{array}{c}18 \\
(11,9)\end{array}$ & $\begin{array}{c}11 \\
(9,1)\end{array}$ & $\begin{array}{c}21 \\
(17,4)\end{array}$ & $18(4)$ & $27(7)$ \\
\hline Gesamt & $\begin{array}{c}115 \\
(100)\end{array}$ & $\begin{array}{r}114 \\
(100)\end{array}$ & $\begin{array}{c}151 \\
(100)\end{array}$ & $\begin{array}{c}151 \\
(100)\end{array}$ & $\begin{array}{c}121 \\
(100)\end{array}$ & $\begin{array}{c}121 \\
(100)\end{array}$ & $\begin{array}{c}393 \\
(100)\end{array}$ & $\begin{array}{c}393 \\
(100)\end{array}$ \\
\hline
\end{tabular}

Tab.14: Vergleich des prä- zum postoperativen Gleason-Score bei unseren Patienten und aktuellen Studien.

Die Tendenz des postoperativen Upgradings ist bei vergleichbaren Studien ebenfalls deutlich erkennbar (Egevad 2001, Lattouf und Saad 2002, Poulus et al. 2005).

Die genaue Analyse der Daten zeigt, dass die präoperative Biopsie den exakten postoperativen Gleason-Score in 34,7\% Fälle voraussagt. In 52\% der Fälle war die präoperative Beurteilung des Malignitätsgrades niedriger als postoperativ, in 13,3\% der Fälle verhielt es sich umgekehrt. Innerhalb von +/-1 Grad nach Gleason konnten 69,4\% der Fälle richtig beurteilt werden.

Gregori et al. fanden bei 289 untersuchten Patienten vergleichbare Ergebnisse. Mit 43,3\% der Fälle, wurde eine etwas höhere Übereinstimmung dokumentiert. Undergraded wurden 40,8\%, overgraded 14,8\% (Gregori et al. 2001). Auch Ege- 
vad und Mitarbeiter kamen mit 45,5\% Übereinstimmungen und 38,8 bzw. 15,7\% Up- bzw. Downgradings zu ähnlichen Resultaten. Eine kanadische Gruppe um Lattouf fand dagegen nur 29,2\% Übereinstimmungen bei 393 untersuchten Patienten. 38,4\% wurden under- gegraded und mit 32,3\% im Vergleich zu unserer Studie relativ viele over-gegraded (Lattouf und Saad 2002).

Unsere Ergebnisse sind limitiert aussagekräftig. Es wurden unterschiedlich viele Stanzen bei den Biopsien entnommen und die Probenentnahme lief nicht nach einem standardisierten Verfahren ab. Ein Teil der fehlenden Übereinstimmung des Gleason-Scores zwischen prä- und postoperativem Befund ist auf Fehler bei der Probenentnahme zurückzuführen (Ruijter et al. 2000). Des Weiteren wurden die histologischen Beurteilungen von verschiedenen Pathologen vorgenommen. Schon Gleason fand heraus, dass nur etwa die Hälfte der Befunde von anderen Pathologen reproduziert werden konnten (Gleason 1992). Es wurden bereits einige Anstrengungen unternommen, um die Variabilität der Beurteilungen beim Gleason-Score zu vermindern (Egevad et al. 2001, Kronz et al. 2000a, Kronz et al. 2000b), doch es wird sicherlich weiterhin unterschiedlichen Meinungen bei den Beurteilungen des Gleason-Scores geben. So wäre eine höhere Übereinstimmung des prä- mit dem postoperativen Gleason-Score bei unseren Patienten wahrscheinlich möglich, wenn nicht verschiedene Pathologen die Gewebe beurteilen würden. Auf einen weiteren wichtigen Punkt machen Aihara et al. und Ruijter et al. aufmerksam. Das Prostatakarzinom hat eine große Heterogenität. Bei mehr als der Hälfte enthalten die Prostatapräparate mindestens 3 verschiedene Gleason-Grade. Derselbe Gleason-Grad findet sich nur in 10-16\% des Gewebes in den Präparaten (Aihara et al. 1994, Ruijter et al. 1996). Dieser Mangel an Repräsentativität der Proben ist offensichtlich ein großes Problem beim präoperativen Grading.

Aufgrund dieser Probleme waren auch bei unseren Patienten nur in 34,7\% der Fälle Übereinstimmungen zu verzeichnen. Es ist also festzuhalten, dass auch der Gleason-Score nur sehr ungenau bestimmt werden konnte und die Übereinstimmungen mit dem postoperativ bestimmten Gleason-Score nur in einem geringen Prozentsatz übereinstimmt. Therapieentscheidungen auf dieser Grundlage sind demnach nicht eindeutig zu treffen. 


\subsection{Konsequenzen der radikalen Prostatektomie}

Welche Komorbiditäten bringt die radikale retropubische Prostatektomie mit sich? In der Literatur werden als Hauptkomplikationen die Inkontinenz und die Impotenz als Folgekomplikationen der Operation angegeben (Sacco et al. 2006, Lepor el al. 2004).

Mittels des Fragebogens wurde im Follow-up der Kontinenzstatus unserer Patienten zu den Zeitpunkten vor und nach der Operation erhoben. Vor der Operation gaben fast alle Patienten an harnkontinent gewesen zu sein. Das postoperative Bild nach einem durchschnittlichen Follow-up von fast 4 Jahren unterscheidet sich signifikant. Nur 20\% der Patienten haben nach eigener Einschätzung ihre Harnkontinenz behalten. Der Rest gab eine Inkontinenz Grad I bis teilweise sogar Grad IV an.

Einige Autoren haben festgestellt, dass die Methode der Datensammlung zur Harninkontinenz zu signifikant unterschiedlichen Ergebnissen führen kann (Talcott et al. 1997, Litwin et al. 1998). Fragt man unsere Patienten, wie häufig in der Literatur üblich, nach der Anzahl der Vorlagen, die sie pro Tag gebrauchen, so zeigt es sich, dass knapp die Hälfte der Patienten keine Vorlagen benötigen. Da die meisten Patienten, die nur eine Vorlage pro Tag benutzen, diese nur zur Sicherheit, nicht aber wegen Harninkontinenz gebrauchen, lassen sich diese klinisch auch als Kontinent definieren (Sacco et al. 2006). Definiert man also den Gebrauch von bis zu einer Vorlage noch als Kontinent, so erfüllen knapp drei Viertel unserer Patienten nach der Umfrage dieses Kriterium. Definitionen der Kontinenz, wie die totale Harnkontrolle oder kein Gebrauch von Vorlagen unterschätzen den, von den Patienten selbst eingeschätzten Kontinenzgrad signifikant (Lepor el al. 2004).

Auch bei uns zeigt sich bei der Definition der Kontinenz nach Vorlagen ein deutlich signifikanter Unterschied zu den nach der Definition der Kontinenzgrade ermittelten Zahlen.

Bezeichnet man also diejenigen Patienten die keine oder nur eine Vorlage täglich brauchen als Kontinent, so verbleiben nach dem durchschnittlichen Follow-up von 46,5 Monaten 27\% der Patienten, die inkontinent sind. Bei diesen Patienten ist 
auch davon auszugehen, dass keine spontane Verbesserung des Kontinenzstatus eintritt.

Die meisten großen Studien zum Kontinenzstatus nach radikaler Prostatektomie weisen bessere Ergebnisse nach. Nach der Definition von keiner oder einer Vorlage pro Tag wurden Inkontinenzraten von 13- 16\% erreicht (Eastham et al. 1996, Sacco el al. 2006, Wei et al. 2000). Zu ähnlichen Ergebnissen kamen Stanford und Mitarbeiter. Hier waren bei ähnlichen Messmethoden 25\% der Patienten inkontinent (Stanford et al. 2000).

Ist eine Verbesserung des Kontinenzstatus im Verlauf nach der Operation zu erwarten? Wenn ja, in welchem Zeitraum ist noch mit einer Verbesserung für die Patienten zu rechnen?

Eine Verbesserung des Kontinenzstatus im postoperativen Verlauf gaben gut zwei Drittel der Patienten an. Durchschnittlich trat diese Verbesserung ein gutes halbes Jahr nach der Operation ein. $12 \%$ der Patienten, die direkt nach der Operation inkontinent waren, konnten innerhalb eines Jahres nach dem Eingriff ihre Harnkontinenz wieder erlangen.

Unsere Analyse wird bestätigt durch die Ergebnisse von anderen Autoren, die eine progressive Verbesserung der Inkontinenzraten innerhalb der ersten 24 Monate nach der Operation beschreiben (Kielb et al. 2001, Wei et al. 2000). Wenn eine Verbesserung des Kontinenzstatus eintritt, dann geschieht dies bei den meisten Patienten innerhalb des ersten Jahres.

Bevor Maßnahmen zur Wiedererlangung der Kontinenz, wie ein künstlicher Sphinkter oder operative Eingriffe, eingeleitet werden, sollte man mindestens 612 Monate nach der Operation abwarten (Tse und Stone 2003). Diese Aussage bestätigen unserer Zahlen. Vor der Einleitung von invasiven Maßnahmen zur Verbesserung einer Inkontinenz nach einer radikalen retropubischen Prostatektomie sollte ein Jahr abgewartet werden.

Die zweite Hauptkomplikation der radikalen retropubischen Prostatektomie ist die Impotenz. Die durch einen validierten Fragebogen ermittelten Ergebnisse unterscheiden in der präoperativen- zu der postoperativen Situation signifikant. So hat- 
ten vor der Operation nahezu alle Patienten keine Potenzprobleme. Nach der Operation haben fast alle Patienten zumeist massive Potenzprobleme angegeben.

Die „American Urological Association“ hat zur erektilen Funktion nach Behandlung eines lokalen Prostatakarzinoms eine Metaanalyse der zwischen 1991 und 2004 veröffentlichten Artikel zu diesem Thema publiziert. Nach radikaler Prostatektomie werde eine komplette erektile Dysfunktion bei 26-100\%, eine partielle bei $16-48 \%$ und intakte erektile Funktion bei $9-86 \%$ beschrieben. Verantwortlich für die sehr uneinheitlichen Ergebnisse seien die sehr uneinheitlichen und schlecht interpretierbaren Bewertungsformen. Einheitliche wissenschaftliche Kriterien seien nötig für die zukünftige Forschung auf diesem Gebiet (Burnett et al. 2007).

\subsection{Konsequenzen und Ausblick}

Es gab in den letzten Jahren viele Bemühungen Nomogramme zu entwickeln, um sowohl den Patienten als auch den behandelnden Ärzten verbesserte Entscheidungshilfen zur Therapieentscheidung an die Hand zu geben (Partin, Kattan, Hamburger Modell usw.). Bei den Nomogrammen werden allerdings zumeist Parameter wie der Gleason-Score, PSA-Wert und Grading abgefragt, die zumindest bei unseren Patienten nur sehr ungenau bestimmt werden konnten. Dies hat zur Folge, dass Scores aus den Nomogrammen ungenau sind und zu falschen Therapieentscheidungen führen können.

Ein Ansatzpunkt, um das richtige Therapieschema für jeden individuellen Patienten zu finden, wäre die Verbesserung der Bildgebung im Rahmen der Diagnostik. Seitz und Mitarbeiter haben in dem Artikel „Bildgebende Verfahren bei der Diagnose des Prostatakarzinoms“ hierzu den aktuellen Stand veröffentlicht (Seitz et al. 2007). Auch Loch hat insbesondere zum computergestützten transrektalen Ultraschall (C-TRUS) einige Arbeiten bereits veröffentlicht (Loch 2007a, Loch 2007b) 


\section{Zusammenfassung}

Zusammenfassend ist also festzustellen, dass die Diagnostik des Prostatakarzinoms durch Biopsien notwendig ist, um festzustellen, ob maligne Zellen vorhanden sind oder nicht. Therapieentscheidungen, die auf weiteren Faktoren wie dem uni- bzw. bilateralen Befall der Drüse, dem Gleason-Score und dem Grading beruhen, sind sehr ungenau und können somit zu falschen Therapieentscheidungen und deren Konsequenzen führen.

Das Spektrum der Therapiemöglichkeiten des lokal begrenzten Prostatakarzinoms ist sehr breit. Von ,active surveillance“, über Brachy- und externe Radiotherapien bis hin zur radikalen Prostatektomie gibt es viele Möglichkeiten, die abhängig von PSA-Werten, Gleason-Score, uni- bzw. bilateralem Seitenlappenbefall und der Entscheidung des Patienten zum Einsatz kommen. Die Konsequenzen für die Lebensqualität und Komorbidität sind je nach Therapie sehr unterschiedlich, so dass die richtige Therapieentscheidung für den Patienten essentiell wichtig für den Verlauf seines Lebens nach der Therapie ist.

Ziel sollte es sein, in Zukunft Therapieentscheidungen treffen zu können, die besser an die Anforderungen einerseits an das Karzinom und andererseits an die Lebensqualität des Patienten angepasst sind. Das Augenmerk sollte hierbei nicht der Ebene der Nomogramme gelten, sondern vielmehr gilt es eine diagnostische Methode zu entwickeln, die genauere Daten zur Ausbreitung und Aggressivität des Karzinoms liefert. 


\section{Anhang}

Fragebogen:

\section{Georg-August-Universität Göttingen}

Stiftung Öffentlichen Rechts - Bereich Humanmedizin-

Medizinische Fakultät

Klinik und Poliklinik für Urologie

Direktor: Prof. Dr. R.-H. Ringert

D-37099 Gottingen

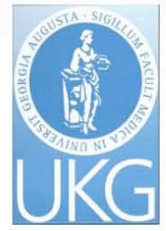

Robert-Koch-Str. 40

37099 Gottingen

Tel. $0551 / 39-6166$

Fax 0551/39-6165

e-mail rhringert@med.uni-

goettingen.de

\section{Fraqebogen für Patienten nach radikaler Prostatektomie:}

Hinweis zum Ausfüllen für Teil 1 und Teil 2 der Fragen:

$\mathrm{Zu}$ jeder der folgenden Frage gibt es mehrere Antwortmöglichkeiten. Bitte wählen Sie die Antwort aus, die Ihre eigene Situation am besten beschreibt und kreuzen die entsprechende Antwort an. Wir möchten Sie bitten, keine Frage auszulassen und jeder Frage nur eine einzige Antwort zuzuordnen.

\section{Fragebogen Teil 1:}

Die willkürliche Kontrolle des Harndrangs ist eine wichtige Funktion, um sich im normalen Alltag wohl zufühlen. Kontinenzstörungen gehören zu den Komplikationen, die nach einer operativen Entfernung der Prostata (radikalen Prostatektomie) auftreten können. Mit den folgenden Fragen möchten wir herausfinden, ob die Operation an ihrer Prostata Ihre Fähigkeit zur Kontrolle des Harndrangs und die damit verbundene Lebensqualität entscheidend beeinflusst hat.

Fragen zu der Situation vor der

Fragen zu der aktuellen Situation: Operation:

Wie oft kommt es bei Ihnen zu

Wie oft kam es bei Ihnen zu unwillkürlichem Harnverlust? unwillkürlichem Harnverlust?

$\square$ Nie

$\square \mathrm{Nie}$

$\square$ Einmal pro Woche oder seltener

$\square$ Einmal pro Woche oder seltener

$\square$ Zwei- bis dreimal pro Woche

$\square$ Zwei- bis dreimal pro Woche

$\square$ Einmal täglich

$\square$ Einmal täglich

$\square$ Mehrmals täglich

$\square$ Mehrmals täglich

$\square$ Ständig

$\square$ Ständig 
Fragen zu der Situation vor der Operation:

Wie hoch war der Harnverlust?

$\square$ Kein Harnverlust

$\square$ Eine geringe Menge

$\square$ Eine mittelgroße Menge

$\square$ Eine große Menge

Wie stark war Ihr Leben durch den Harnverlust beeinflusst?

$\begin{array}{llll}0 \square \quad 1 \square \quad 2 \square \quad 3 \square & 4 \square \quad 5 \square \\ \text { gar nicht } & & & \\ 6 \square \quad 7 \square \quad 8 \square \quad 9 \square & 10 \square \\ & & & \text { stark }\end{array}$

Wann kam es zu Harnverlust?

$\square$ Zu keiner Zeit

$\square$ Beim Husten, Niesen, Lachen usw.

$\square$ Bei leichter körperlicher Anstrengung, wie z.B. Treppensteigen, Gehen usw.

$\square$ Unkontrollierbarer Harnabgang im Stehen und Liegen

$\square$ Harnverlust trat ständig auf
Fragen zu der aktuellen Situation:

Wie hoch ist der Harnverlust?

$\square$ Kein Harnverlust

$\square$ Eine geringe Menge

$\square$ Eine mittelgroße Menge

$\square$ Eine große Menge

Wie stark ist Ihr Leben durch den Harnverlust beeinflusst?

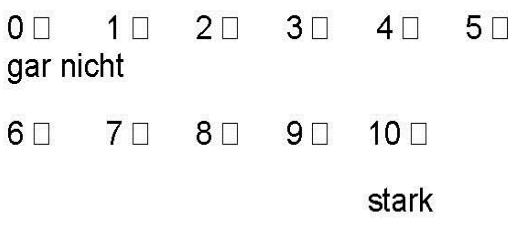

Wann kommt es zu Harnverlust?

$\square$ Zu keiner Zeit

$\square$ Beim Husten, Niesen, Lachen usw.

$\square$ Bei leichter körperlicher Anstrengung wie z.B. Treppensteigen, Gehen usw.

$\square$ Unkontrollierbarer Harnabgang im Stehen und Liegen

$\square$ Harnverlust trat ständig auf 
Fragen zu der Situation vor der Operation:

Wie viele Vorlagen benutzten Sie pro 24 Stunden?

$\square$ Keine

$\square 1$ Vorlage

$\square 2$ Vorlagen

$\square 3$ Vorlagen

$\square 4$ Vorlagen oder mehr

Falls Sie inkontinent (=unwillkürlicher Harnabgang) waren, hatten Sie etwas unternommen um die Situation zu verbessern?

$\square$ Ich war nicht Inkontinent

$\square$ Nein, ich habe nichts unternommen

$\square \mathrm{Ja}$, ich habe Tabletten eingenommen (z.B. Alna $®$, Omnic $®$, Spasmex $®$, Spasmolyt $($, Detrusitol $($, Oxybutynin $($ ) oder andere)

$\square \mathrm{Ja}$, ich hatte einen suprapubischen Dauerkatheter (Abfluss des Urin durch einen Schlauch durch die Bauchdecke)

$\square$ Ja, ich wurde operiert (z.B. SphinkterImplantation)
Fragen zu der aktuellen Situation:

Wie viele Vorlagen benutzen Sie pro 24 Stunden?

$\square$ Keine

$\square 1$ Vorlage

$\square 2$ Vorlagen

$\square 3$ Vorlagen

$\square 4$ Vorlagen oder mehr

Falls Sie inkontinent (=unwillkürlicher Harnabgang) sind, haben Sie etwas unternommen um die Situation zu verbessern?

$\square$ Ich bin nicht Inkontinent

$\square$ Nein, ich habe nichts unternommen

$\square \mathrm{Ja}$, ich habe Tabletten eingenommen (z.B. Alna ${ }^{\circledR}$, Omnic $®$, Spasmex $®$, Spasmolyt $($, Detrusitol $($, Oxybutynin $\circledast$ oder andere)

$\square \mathrm{Ja}$, ich habe einen suprapubischen Dauerkatheter (Abfluss des Urin durch einen Schlauch durch die Bauchdecke)

$\square$ Ja, ich wurde operiert (z.B. SphinkterImplantation)

Ist Ihre Kontrolle über das Wasserlassen (Kontinenz) seit der Operation besser geworden als zum jetzigen (aktuellen) Zeitpunkt?

$\square$ Nein, die Situation ist unverändert

$\square \mathrm{Ja}$, nach der Operation war meine Kontrolle des Wasserlassens schlechter. Nach welchem Zeitraum hat sich die Situation gebessert? Ca. ..... Monat(e) nach der Operation 


\section{Fraqebogen Teil 2:}

Sexualität ist ein wichtiger Teil des gesamten körperlichen und seelischen Wohlbefindens. Erektionsstörungen gehören zu den Komplikationen, die nach einer operativen Entfernung der Prostata (radikalen Prostatektomie) auftreten können. Mit den folgenden Fragen möchten wir herausfinden, ob die Operation an Ihrer Prostata ihre Erektionsfähigkeit entscheidend beeinflusst hat.

Fragen zu der Situation vor der Operation:

Wie zuversichtlich waren Sie, eine Erektion zu bekommen und aufrechterhalten zu können?

$\square$ Sehr wenig

$\square$ Wenig

$\square$ Unentschieden

$\square$ Ziemlich

$\square$ Sehr

Wenn Sie bei sexueller Stimulation Erektionen hatten, wie oft waren Ihre Erektionen hart genug, um in Ihre Partnerin einzudringen?

$\square$ Keine sexuellen Aktivitäten

$\square$ Fast nie oder nie

$\square$ Selten (viel weniger als die Hälfte der Versuche)

$\square$ Manchmal (etwa die Hälfte der Versuche)

$\square$ Meistens (viel mehr als die Hälfte der Versuche)

$\square$ Fast immer oder immer
Fragen zu der aktuellen Situation:

Wie zuversichtlich sind Sie, eine Erektion zu bekommen und aufrechterhalten zu können?

$\square$ Sehr wenig

$\square$ Wenig

$\square$ Unentschieden

$\square$ Ziemlich

$\square$ Sehr

Wenn Sie bei sexueller Stimulation Erektionen haben, wie oft ist Ihre Erektionen hart genug, um in Ihre Partnerin einzudringen?

$\square$ Keine sexuellen Aktivitäten

$\square$ Fast nie oder nie

$\square$ Selten (viel weniger als die Hälfte der Versuche)

$\square$ Manchmal (etwa die Hälfte der Versuche)

$\square$ Meistens (viel mehr als die Hälfte der Versuche)

$\square$ Fast immer oder immer 
Fragen zu der Situation vor der Operation:

Wie oft waren Sie beim

Geschlechtsverkehr in der Lage, die Erektion aufrechtzuerhalten, nachdem Sie in Ihre Partnerin eingedrungen waren?

$\square$ Ich habe keinen Geschlechtsverkehr versucht

\section{$\square$ Fast nie oder nie}

$\square$ Selten (viel weniger als die hälfte der Versuche)

$\square$ Manchmal (etwa die hälfte der Versuche)

$\square$ Meistens (viel mehr als die hälfte der Versuche)

$\square$ Fast immer oder immer

Wenn Sie versuchten,

Geschlechtsverkehr zu haben, wie oft war er befriedigend für Sie?

$\square$ Ich habe keinen Geschlechtsverkehr versucht

\section{$\square$ Fast nie oder nie}

$\square$ Selten (viel weniger als die hälfte der Versuche)

$\square$ Manchmal (etwa die hälfte der Versuche)

$\square$ Meistens (viel mehr als die hälfte der Versuche)

$\square$ Fast immer oder immer
Fragen zu der aktuellen Situation:

Wie oft sind Sie beim

Geschlechtsverkehr in der Lage, die Erektion aufrechtzuerhalten, nachdem Sie in Ihre Partnerin eingedrungen sind?

$\square$ Ich habe keinen Geschlechtsverkehr versucht

$\square$ Fast nie oder nie

$\square$ Selten (viel weniger als die hälfte der Versuche)

$\square$ Manchmal (etwa die hälfte der Versuche)

$\square$ Meistens (viel mehr als die hälfte der Versuche)

$\square$ Fast immer oder immer

Wenn Sie versuchen,

Geschlechtsverkehr zu haben, wie oft ist er befriedigend für Sie?

$\square$ Ich habe keinen Geschlechtsverkehr versucht

$\square$ Fast nie oder nie

$\square$ Selten (viel weniger als die hälfte der Versuche)

$\square$ Manchmal (etwa die hälfte der Versuche)

$\square$ Meistens (viel mehr als die hälfte der Versuche)

$\square$ Fast immer oder immer 
Fragen zu der Situation vor der Operation:

Wie schwierig war es beim Geschlechtsverkehr, die Erektion bis zum Ende des Geschlechtsverkehrs aufrechtzuerhalten?

$\square$ Ich habe keinen Geschlechtsverkehr versucht

\section{$\square$ Äußerst schwierig \\ $\square$ Sehr schwierig \\ $\square$ Schwierig \\ $\square$ Ein bisschen schwierig \\ $\square$ Nicht schwierig}

Haben Sie irgendwelche Hilfsmittel verwendet, um eine Erektion zu bekommen und/oder diese aufrechtzuerhalten?

$\square$ Nein, ich habe keine Hilfsmittel verwendet

$\square \mathrm{Ja}$, ich habe Tabletten eingenommen ( z.B. Viagra $\otimes$, Cialis $\otimes$, Levitra $\otimes$ oder andere)

$\square \mathrm{Ja}$, ich habe mechanische Hilfsmittel verwendet (z.B. Vakuumpumpe, Penisring oder andere)

$\square \mathrm{Ja}$, ich habe Hilfsmittel verwendet, die als Antwortmöglichkeit nicht aufgeführt wurden. Welche?
Fragen zu der aktuellen Situation:

Wie schwierig ist es beim

Geschlechtsverkehr, die Erektion bis zum Ende des Geschlechtsverkehrs aufrechtzuerhalten?

$\square$ Ich habe keinen Geschlechtsverkehr versucht

$\square$ Äußerst schwierig

$\square$ Sehr schwierig

$\square$ Schwierig

$\square$ Ein bisschen schwierig

$\square$ Nicht schwierig

Verwenden Sie irgendwelche Hilfsmittel, um eine Erektion zu bekommen und/oder diese aufrechtzuerhalten?

$\square$ Nein, ich verwende keine Hilfsmittel $\square$ Ja, ich nehme Tabletten ein ( $z$.B. Viagra $\circledast$, Cialis $\Theta$, Levitra $\circledast$ oder andere)

$\square \mathrm{Ja}$, ich verwende mechanische Hilfsmittel ( Vakuumpumpe, Penisring oder andere)

$\square$ Ja, ich verwende Hilfsmittel, die als Antwortmöglichkeit nicht aufgeführt wurden. Welche?

War Ihre Erektionsfähigkeit nach der Operation schlechter als zum jetzigen (aktuellen) Zeitpunkt?

$\square$ Keine sexuellen Aktivitäten

$\square$ Nein, die Situation ist unverändert

$\square$ Ja, meine Erektion war nicht ausreichend, um befriedigenden Geschlechtsverkehr zu haben. Nach welchem Zeitraum hat sich die Situation gebessert? Ca. ...... Monat(e) nach der Operation. 


\section{Fraqebogen Teil 3:}

Im Folgenden noch weitere Fragen zu Ihrer Situation nach der Operation: Bitte versuchen Sie eine möglichst genaue Antwort zu geben. Fragen, die Sie nicht beantworten können, lassen Sie bitte einfach aus.

In welchen Zeitabständen begeben Sie sich seit der Operation zur Nachsorge in ärztliche Behandlung?

$\square$ Ca. alle Monate

$\square$ Unregelmäßig (ungefähr ..... Mal pro Jahr)

Wann waren Sie zuletzt zur Nachsorge in ärztlicher Behandlung?

Monat: Jahr:

Name des Hausarztes/ Urologen:

Erhielten Sie direkt nach der Operation eine zusätzliche Behandlung?

$\square$ Nein

$\square \mathrm{Ja}$, eine medikamentöse Behandlung mit folgenden Tabletten: ...(Z.B. Casodex® oder anderen). Seit wann?.

$\square \mathrm{Ja}$, eine medikamentöse Behandlung mit folgenden Spritzen

(z.B. Trenantone $\otimes$, Zoladex $®$ oder anderen)

$$
\begin{aligned}
& \square \text { jeden Monat } \\
& \square \text { alle } 3 \text { Monate }
\end{aligned}
$$

Seit wann?

$\square \mathrm{Ja}$, Bestrahlung. Seit wann?

$\square \mathrm{Ja}$, eine Chemotherapie (Taxotere $\Theta$, Docetaxel $₫$, Estramustin $\circledast$, Mitoxantrane $₫$

$\square \mathrm{Ja}$, ich wurde erneut operiert. Wann? (Tag/Monat/Jahr)

Ist Ihnen ihr letzter PSA-Wert bekannt?

$\square$ Nein

$\square$ Ja, der PSA-Wert beträgt:

Bestimmt an folgendem Datum: 


\section{Literaturverzeichnis}

Abbou CC, Salomon L, Hoznek A (2000): Laparoscopic radical prostatectomy: Preliminary results. Urology 55, 630-634

Aihara M, Wheeler TM, Ohori M, Scardino PT (1994): Heterogenity of prostate cancer in radical prostatectomy specimens. Urology $\underline{43}, 60-66$

Alschibaja M, Wegner M, Massmann J, Funk A, Hartung R, Paul R (2005): Prostate cancer volume - can it be predicted preoperatively? Urol Int 75,354-359

Ansell JS (1959): Radical transvesical prostatectomy: preliminary report on an approach to surgical excision of localized prostate malignacy. J Urol 83, 373

Armbruster DA (1993): Prostate-specific antigen: Biochemestry, analytical methods, and clinical application. Clin Chem 39, 181-195

Aus G, Abbou CC, Bolla M, Heidenreich A, van Poppel H, Schmid H-P, Wolff JM, Zattoni F: Guidelines on prostate cancer; hrsg. von der European Association of Urology, Arnheim 2005, 30-34

Bas AJ, Verhage \& Lambertus ALM, Kiemeney (2003): Inherited predisposition to prostate cancer. Eur J Epidemiol 18, 1027-1038

Belt E, Ebert CE, Surber AC Jr (1939): A new anatomic approach in perineal prostatectomy. J Urol $\underline{41}, 482-497$ 
Bertz J, Giersiepen K, Haberland J, Hentschel S, Kaatsch P, Katalinic A, Stabenow R, Stegmaier C, Ziegler H: Krebs in Deutschland; hrsg. von: Gesellschaft der epidemiologischen Krebsregister in Deutschland e.V. und das Robert-KochInstitut, 5. überarbeitete, aktualisierte Ausgabe, Saarbrücken 2006, 68-71

Bostwick DG, Bostwick KC (2004) 'Vanishing' prostate cancer in radical prostatectomy specimens: incidence and long-term follow-up in 38 cases. BJU Int $\underline{94}$, $57-58$

Burnett AL, Aus G, Canby-Hagino ED, Cookson MS, D'Amico AV, Dmochowski RR, Eton DT, Forman JD, Goldenberg SL, Hernandez J, Higano CS, Kraus S, Liebert M, Moul JW, Tangen C, Thrasher JB, Thompson I, AUA Prostate Cancer Guideline Update Panel (2007): Erectile function outcome reporting after clinically localized prostate cancer treatment. J Urol 178, 597-601

Campbell EW (1959): Total prostatectomy with preliminary ligation of the vascular pedicles. J Urol $\underline{81}, 464$

Carter HB, Partin AW: Diagnosis and staging of prostate cancer. In: Campbell's urology; hrsg. von Walsh PC, Retik AB, Darracott Vaughan E, Wein AJ, 8.Auflage; Saunders, Philadelphia, London, New York, St. Louis, Sydney, Toronto $2002,3055-3079$

Catalona WJ (2003): Informed consent for prostate-specific antigen screening. Urology $\underline{61}, 17-19$

Catalona WJ, Ramos CG, Carvalhal GF, Yan Y (2000): Lowering PSA cutoffs to enhance detection of curable prostate cancer. Urology $\underline{55}$ (6), 791-795 
Connolly SS, O’Malley KJ, O’Brien A, Kelly DG, Mulvin DW, Quinlan, DM (2004): Can prostate biopsies predict suitability for nerve-sparing radical prostatectomy? Scand J Urol Nephrol 38, 216-220

Coogan CL, Latchamsetty KC, Greenfield J, Corman JM, Lynch B, Porter CR (2005): Increasing the number of biopsy cores improves the concordance of biopsy Gleason score to prostatectomy Gleason score. BJU Int 96, 324-327

Denis LJ, Murphy GP, Schröder FH (1995): Report of the Consensus Workshop on Screening and Global Strategy for Prostate Cancer. Cancer 75, 1187-1207

Djavan B, Margreiter M (2007): Biopsy standards for detection of prostate cancer. World J Urol 25, 11-17

Eastham JA, Scardino PT: Radical prostatectomy for clinical stage T1 and T2 prostate cancer. In: Comprehensive textbook of genitourinary oncology; hrsg. von Vogelzang NJ, Scardino PT, Shipley WU, Coffey DS, 1.Auflage; Williams and Wilkins, Baltimore, Philadelphia, Hong Kong, London, Munich, Sydney, Tokyo $1996,741-757$

Eastham JA, Kattan MW, Rogers E, Goad JR, Ohori M, Boone TB, Scardino PT(1996): Risk factors for urinary incontinence after radical prostatectomy. J Urol $\underline{156}, 1707-1713$

Eastham JA, Carver B, Katz J, Kattan MW(2002): Clinical stage T1c prostate cancer: pathologic outcomes following radical prostatectomy in black and white men. Prostate 50, 236-240.

Egevad L (2001): Reproducibility of Gleason grading of prostate cancer can be improved by the use of reference images. Urology 57, 291-295 
Egevad L, Norlén BJ, Norberg M (2001): The value of multiple core biopsies for predicting the Gleason score of prostate cancer. BJU Int $\underline{88}, 716-721$

Eskocorapci SY, Baydar DE, Akbal C, Sofikerim M, Günay M, Ekici S, Ozen H (2004): An extended 10-core transrectal ultrasonography guided prostate biopsy protocol improves the detection of prostate cancer. Eur Urol 45, 444-448

Fink KG, Hutarew G, Pytel A, Esterbauer B, Jungwirth A, Dietze O, Schmeller NT (2003): One 10-core prostate biopsy is superior to two sets of sextant prostate biopsies. BJU Int 92, 385-388

Fischer C, Schreiber A, Altwein JE (2005): Long-term outcome of radical prostatectomy in an observation period of at least 10 years. Aktuelle Urol $\underline{36}, 131-135$

Frohmüller H, Wirth M: Die radikale Prostatektomie. In: Aktuelle Therapie des Prostatakarzinoms; hrsg. von Ackermann R, Altwein JE, Faul P; Springer Verlag, Berlin, Heidelberg 1991, 101-121

Frohmüller H, Theiß M: Prostata-Karzinom. In: Prostataerkrankungen im höheren Lebensalter; hrsg. von Frohmüller H, Theiß M, Bracher F, 2.Auflage; Wissenschaftlicher Verlag, Stuttgart 2002, 145-210

Gibbons RP: Radical perineal prostatectomy. In: Campbell's urology; hrsg. von Walsh PC, Retik AB, Darracott Vaughan E, Wein AJ, 8.Auflage; Saunders, Philadelphia, London, New York, St. Louis, Sydney, Toronto 2002, 3131-3145

Gleason DF (1992): Histologic grading of prostate cancer: a perspective. Human Pathol 23, 273-279 
Goldstein NS, Bégin LR, Grody WW, Novak JM, Qian J, Bostwick DG (1995): Minimal or no cancer in radical prostatectomy specimens: report of 13 cases of the "vanishing cancer phenomenon'. Am J Surg Pathol 19, 1002-1009

Gore JL, Shariat SF, Miles BJ, Kadmon D, Jiang N, Wheeler TM, Slawin KM (2001): Optimal combinations of systematic sextant and laterally directed biopsies for the detection of prostate cancer. J Urol 165, 1554-1559

Gregori A, Vieweg J, Dahm P, Paulson DF (2001): Comparison of ultrasoundguided biopsies and prostatectomy specimens: predictive accuracy of Gleason score and tumor site. Urol Int $\underline{66}, 66-71$

Grossklaus DJ, Coffey CS, Shappell SB, Jack GS, Cookson MS (2001): Prediction of tumour volume and pathological stage in radical prostatectomy specimens is not improved by taking more prostate needle-biopsy cores. BJU Int $\underline{88}, 722-726$

Guillonneau B, Vallancien G (2000): Laparoscopic radical prostatectomy: The Montsouris experience. J Urol 163, 418-422

Han M, Partin AW, Chan DY, Walsh PC (2004): An evaluation of the decreasing incidence of positive surgical margins in a large retropubic prostatectomy series. $\mathrm{J}$ Urol 171, 23-26

Helpap B (1998): Differential diagnosis of glandular proliferations in the prostate. A convential and immunohistochemical approach. Virchows Arch $\underline{433}, 397-405$ 
Korfage IJ, Essink-Bot ML, Borsboom GJJM, Madalinska JB, Kirkels WJ, Habbema JDF, Schröder FH, de Koning HJ (2005): Five-year follow-up of healthrelated quality of life after primary treatment of localized prostate cancer. Int $\mathbf{J}$ Cancer 116, 291-296

Herkommer K, Kuefer R, Gschwend JE, Hautmann RE, Volkmer BG (2004) Pathological T0 prostate cancer without neoadjuvant therapy: clinical presentation and follow-up. Eur Urol 45, 36-41

Hodge KK, McNeal JE, Terris MK, Stamey TA (1989): Random systematic versus directed ultrasound guided transrectal core biopsies of the prostate. J Urol 142, $71-74$

Hull GW, Rabbani F, Abbas F, Wheeler TM, Kattan MW, Scardino PT (2002): Cancer control with radical prostatectomy alone in 1,000 consecutive patients. $\mathrm{J}$ Urol 167, 528-534

Kielb S, Dunn RL, Rashid MG, Murray S, Sanda MG, Montie JE, Wei JT (2001): Assessment of early continence recovery after radical prostatectomy: patient reported symptoms and impairment. J Urol 166, 958-961

Kronz JD, Silberman MA, Allsbrook WC, Epstein JI (2000a): A web-based tutorial improves practicing pathologists' Gleason grading of images of prostate carcinoma specimens obtained by needle biopsy. Cancer $\underline{89}, 1818-1823$

Kronz JD, Silberman MA, Allsbrook WC Jr (2000b): Pathology residents' use of a Web-based tutorial to improve Gleason grading of prostate carcinoma on needle biopsies. Hum Pathol 31, 1044-1050

Kuchler H (1866): Über Prostatavergrösserung. Dtsch Klin 18, 458 
Kundu, Shilajit D, Roehl, Kimberly A, Eggener, Scott E; Antenor, Jo Ann V; Han, Misop; Catalona, William J (2004): Potency, continence and complications in 3,477 consecutive radical retropubic prostatectomies. J Urol 172, 2227-2231

Lattouf JB, Saad F (2002): Gleason score on biopsy: is it reliable for predicting the final grade on pathology? BJU Int $\underline{90}, 694-698$

Leandri P, Rossignol G, Gautier J-R, Ramon J (1992): Radical retropubic prostatectomy: Morbidity and quality of life. Experience with 620 consecutive cases. J Urol 147, 883-887

Lepor H, Kaci L, Xue X (2004): Continence following radical retropubic prostatectomy using self-reporting instruments. J Urol 171, 1212-1215

Litwin MS, Lubeck DP, Henning JM, Carroll PR (1998): Differences in urologist and patient assessment of health related quality of life in men with prostate cancer: results of the CaPSURE database. J Urol 159, 1988-1992

Loch T (2007a): Urologic imaging for localized prostate cancer in 2007. World J Urol 25, 121-129

Loch T (2007b): Computerized transrectal ultrasound (C- TRUS) of the prostate: detection of cancer in patients with multiple negative systematic random biopsies. World J Urol 25, 375-380

Matsubara A, Mutaguchi K, Yasumoto H, Takeshima Y, Teishima J, Seki M, Hasegawa Y, Usui T (2006): Transrectal ultrasound-guided 10-core biopsy of the prostate for Japanese patients. Hiroshima J Med Sci 55, 65-70 
Millin T (1947): Retropubic urinary surgery. Williams and Wilkins, Baltimore (Eastham JA, Scardino PT: Radical prostatectomy for clinical stage T1 and T2 prostate cancer. In: Comprehensive textbook of genitourinary oncology; hrsg. von Vogelzang NJ, Scardino PT, Shipley WU, Coffey DS, 1.Auflage; Williams and Wilkins, Baltimore, Philadelphia, Hong Kong, London, Munich, Sydney, Tokyo 1996, 741-757)

Mostofi FK, Sesterhenn I, Sobin LH (1980): Histological typing of prostate tumors. International classifikation of tumors. World health organisation, Geneva, Nr.22

Nöske HD, Breiwieser P (1973): Zur Geschichte der Prostatachirurgie. Münch Med Wochenschr 115, 1194-1198

Obek C, Louis P, Civantos F, Soloway MS (1999): Comparison of digital rectal examination and biopsy results with the radical prostatectomy specimen. J Urol 161, 494-498

Oesterling JE, Jacobsen SJ, Chute CG, Guess HA, Girman CJ, Panser LA, Lieber MM (1993): Serum prostate-specific antigen in a community-based population of healthy men. Establishment of age-specific reference ranges. JAMA 270, 860-864

Partin AW, Mangold LA, Lamm DM, Walsh PC, Epstein JI, Pearson JD (2001): Contemporary update of the prostate cancer staging nomograms (Partin tables) for the new millennium. Urology $\underline{58}, 843-848$

Paul R, Schöler S, van Randenborgh H, Kübler H, Alschibaja M, Busch R, Hartung R (2005): Optimization of prostatic biopsy: a prospective randomized 
trial comparing the sextant biopsy with a 10-core biopsy. Impact of prostatic region of sampling. Urol Int 74, 203-208

Penson DF, Litwin MS, Aaronson NK (2003): Health related quality of life in men with prostate cancer. J Urol 169, 1653-1661

Poulus CK, Daggy JK, Cheng L (2005): Preoperative prediction of Gleason grade in radical prostatectomy specimens: the influence of different Gleason grades from multiple positive biopsy sites. Mod Pathol 18, 228-234

Preston DM, Levin LI, Jacobson DJ, Jacobson SJ, Rubertone M, Holmes E, Murphy GP, Moul JW (2000): Prostate-specific antigen levels in young white and black men 20 to 45 years old. Urology $\underline{56}, 812-816$

Reiner WG, Walsh PC (1979): An anatomical approach to the surgical managment of the dorsal vein and Santorini's plexus during radical retropubic surgery. J Urol 121, 198-200

Reiter RE, deKernion JB: Epidemiology, etiology, and prevention of prostate cancer. In: Campbell's urology; hrsg. von Walsh PC, Retik AB, Darracott Vaughan E, Wein AJ, 8.Auflage; Saunders, Philadelphia, London, New York, St. Louis, Sydney, Toronto 2002, 3003-3024

Rifkin MD (1998): Prostate cancer: the diabnostic dilemma and the place of imaging in detection and staging. World J Urol 16, 76-80

Rosen RC (1999): Development and evaluation of an abridged, 5-item version of the International Index of Erectile Functin (IIEF-5) as a diagnostic tool for erectile dysfunction. Int J Impotence 11, 319-326 
Roumeguere T, Bollens R, Bossche MV, Rochet D, Bialek D, Hoffmann P, Quackels T, Damoun A, Wespes E, Schulman CC, Zlotta AR (2003): Radical prostatektomy: a prospective comparison of oncological and functional results between open an laparocopic approaches. World J Urol 20, 360-366

Ruijter ET, van de Kaa CA, Schalken JA, Debruyne FM, Ruiter DJ (1996): Histological grade heterogeneity in multifocal prostate cancer. Biological and clinical implications. J Pathol 180, 295-299

Ruijter E, van Leenders G, Miller G, Debruyne F, van De Kaa C (2000): Errors in histological grading by prostatic needle biopsy specimens: frequency and predisposing factors. J Pathol 192, 229-233

Sacco E, Prayer-Galetti T, Pinto F, Fracalanca S, Betto G, Pagano F, Artibani W (2006): Urinary incontinence after radical prostatectomy: incidence by definition, risk factors and temporal trend in a large series with a long-term follow-up. BJU Int $\underline{97}, 1234-1241$

Salomon L, Colombel M, Patard JJ, Gasman D, Chopin D, Abbou CC (1997): Prostate Biopsy in the staging of prostate cancer. Prostate Cancer Prostatic Dis $\underline{1}, 54-58$

Scales CD Jr, Presti JC Jr, Kane CJ, Terris MK, Aronson WJ, Amling CL, Freedland SJ (2007): Predicting unilateral prostate cancer based on biopsy features: implications for focal ablative therapy--results from the SEARCH database. J Urol 178, 1249- 52

Schmitz-Dräger BJ, Ebert T, Hartmann KA, Beiche B, Anastasiadis AG: Prostatakarzinom. In: Therapiekonzepte Onkologie; hrsg. von Seeber S, Schütte J, 4. Auflage; Springer, Berlin, Heidelberg, New York, Hongkong, London, Mailand, Paris, Tokio 2003, 1244-1267 
Schuessler WW, Schulam PG, Clayman RV, Kavoussi LR (1997): Laparoscopic radical prostatectomy: initial short-term experience. Urology 50, 854-857

Seitz M, Scher B, Scherr M, Tilki D, Schlenker B, Gratzke C, Schipf A, Stinislaus P, Müller-Lisse U, Reich O, Stief C (2007): Bildgebende Verfahren bei der Diagnose des Prostatakarzinoms. Urologe $\underline{46}, 1435-1448$

Shah O, Robbins DA, Melamed J, Lepor H (2003): The New York University nerve sparing algorithm decreases the rate of positive surgical margins following radical retropubic prostatektomy. J Urol 169, 2147-2152

Sofer M, Hamilton-Nelson KL, Civantos F, Soloway MS (2002): Positive surgical margins after radical retropubic prostatectomy: the influence of site and number on progression. J Urol 167, 2453-2456

Srigley JR: No cancer in the radical prostatectomy specimen: an approach to a surgical pathologist's dilemma. In: Microtutorial: a systematic approach to prostate needle biopsies. Canadian Association of Pathologists Annual Meeting. Prince Edward Island, Charlottetown 2003, Section V: 32-36

Stanford JL, Feng Z, Hamilton AS, Gilliland FD, Stephenson RA, Eley JW, Albertsen PC, Harlan LC, Potosky AL (2000): Urinary and Sexual Function After Radical Prostatectomy for Clinically Localized Prostate Cancer. JAMA 283, 354360

Swindle P, Eastham JA, Ohori M, Kattan MW, Wheeler T, Maru N, Slawin K, Scardino PT (2005): Do margins matter? The prognostic significance of positive surgical margins in radical prostatectomy specimens. J Urol 174, 903-907 
Szymanowski J, Dudek P, Ratajski J, Wysocki M (2002): Correlations among prostatic-specific antigen, Gleason score, staging and grading in patients after radical prostatectomy. BJU Int $\underline{89}, 612-613$

Talcott JA, Rieker P, Propert KJ, Clark JA, Wishnow KI, Loughlin KR, Richie JP, Kantoff PW (1997): Patient-reported impotence and incontinence after nervesparing radical prostatectomy. J Natl Cancer Inst $\underline{89}$, 1117-1123

Trpkov K, Gao Y, Hay R, Yimaz A (2006) No residual cancer on radical prostatectomy after positive 10-core biopsy: incidence, biopsy findings, and DNA specimen identity analysis. Arch Pathol Lab Med 130, 811-816

Tse V, Stone AR (2003): Incontinence after prostatectomy: the artificial urinary sphincter. BJU Int 92, 886-889

Walsh PC: Anatomic radical retropubic prostatectomy. In: Campbell's urology; hrsg. von Walsh PC, Retik AB, Darracott Vaughan E, Wein AJ, 8.Auflage; Saunders, Philadelphia, London, New York, St. Louis, Sydney, Toronto 2002, 3107 3128

Walsh PC, Donker PJ (1982): Impotence following radical prostatectomy: insight into etiology and prevention. J Urol $\underline{128}, 492-497$

Wang MC. Valenzuela LA, Murphy GP, Chu TM (1979): Purification of a human prostate specific antigen. Invest Urol 17, 159-163

Weckermann D, Holl G, Dorn R, Wagner T, Harzmann R (2007): Reliability of preoperative diagnostics and location of lymph node metastases in presumed unilateral prostate cancer. BJU Int 99, 1036-1040 
Wei JT, Dunn RL, Marcovich R, Montie JE, Sanda MG (2000): Prospective assessment of patient reported urinary continence after radical prostatectomy. J Urol $\underline{164}, 744-748$

Winfield HN, Cadeddu JA: Other applications of laparoscopic surgery. In: Campbell's urology; hrsg. von Walsh PC, Retik AB, Darracott Vaughan E, Wein AJ, 8.Auflage; Saunders, Philadelphia, London, New York, St. Louis, Sydney, Toronto $2002,3687-3703$

Young HH (1905): The early diagnosis and radical cure of carcinoma of the prostate. J Urol (2002) 168, 914-921

Zynger DL, Dimov ND, Yang XJ (2006) Trends in carcinoma volume in 1612 radical prostatectomies: increased evidence of minimal and no residual cancer. Mod Pathol 19 (Suppl 3): 90A 


\section{Danksagung}

Herrn Dr. med. Arne Strauss und Herrn Dr. med. Thorsten Seiler danke ich für das freundliche Überlassen des Themas der vorliegenden Dissertation sowie die sehr zügige und sorgfältige Durchsicht und Korrektur des Manuskriptes.

Mein besonderer Dank gilt Herrn Dr. med. Arne Strauss für die herausragende und enorm engagierte Betreuung meines Dissertationsvorhabens. Er konnte mir in einigen schwierigen Phasen stets schnell und spontan weiterhelfen, sowie allen Mitarbeitern der Abteilung Urologie für die angenehme Arbeitsatmosphäre und die schnelle und unbürokratische Unterstützung bei kleineren und größeren Anliegen. 


\section{Lebenslauf}

Als Sohn des Gynäkologen Dr. med. Thomas Rogalli und der Lehrerin Marlies Rogalli, geb. Dessel, wurde ich, Gabriel Alexander Rogalli, am 17. Dezember 1979 in Herdecke geboren.

Von 1986 bis 1999 besuchte ich die Schule nach der Pädagogik von R. Steiner in Witten/Annen, an der ich schließlich die allgemeine Hochschulreife erlangte.

Es folgte der einjährige Zivildienst in der Pflege des Gemeinschaftskrankenhauses Herdecke.

Im Sommersemester 2001 begann ich das Studium der Humanmedizin an der Georg-August-Universität zu Göttingen. Im April 2003 absolvierte ich die ärztliche Vorprüfung, im April 2004 folgte der erste Abschnitt der ärztlichen Prüfung (alte AO). Im Wintersemester 2006/07 widmete ich mich der vorliegenden Dissertation.

Ab Februar 2007 absolvierte ich mein Praktisches Jahr am Instituto Nacional de Cancerolgía in Mexiko City (Chirurgie), am Diakonissenkrankenhaus in Bremen (Innere) und in der Allgemeinarztpraxis Dr.med. Kracht in Goslar (Wahlfach Allgemeinmedizin).

Im Juni 2008 absolvierte ich den Zweiten Abschnitt der ärztlichen Prüfung (neue AO) und erlangte meine Approbation als Arzt.

Seit September 2008 arbeite ich in der Abteilung für Innere Medizin des Krankenhauses St.-Joseph-Stift Bremen als Assistenzarzt. 